\title{
$q$-HYPERGEOMETRIC SOLUTIONS OF QUANTUM DIFFERENTIAL EQUATIONS, QUANTUM PIERI RULES, AND GAMMA THEOREM
}

\author{
VITALY TARASOV ${ }^{\circ}$ AND ALEXANDER VARCHENKO*
}

*Department of Mathematics, University of North Carolina at Chapel Hill Chapel Hill, NC 27599-3250, USA

${ }^{\star}$ Faculty of Mathematics and Mechanics, Lomonosov Moscow State University Leninskiye Gory 1, 119991 Moscow GSP-1, Russia

-Department of Mathematical Sciences, Indiana University-Purdue University Indianapolis 402 North Blackford St, Indianapolis, IN 46202-3216, USA

- St. Petersburg Branch of Steklov Mathematical Institute

Fontanka 27, St. Petersburg, 191023, Russia

Key words: Flag varieties, quantum differential equation, dynamical connection, $q$-hypergeometric solutions

2010 Mathematics Subject Classification: 82B23, 17B80, 14N15, 14N35

\begin{abstract}
We describe $q$-hypergeometric solutions of the equivariant quantum differential equations and associated $q K Z$ difference equations for the cotangent bundle $T^{*} \mathcal{F}_{\boldsymbol{\lambda}}$ of a partial flag variety $\mathcal{F}_{\boldsymbol{\lambda}}$. These $q$-hypergeometric solutions manifest a Landau-Ginzburg mirror symmetry for the cotangent bundle. We formulate and prove Pieri rules for quantum equivariant cohomology of the cotangent bundle. Our Gamma theorem for $T^{*} \mathcal{F}_{\boldsymbol{\lambda}}$ says that the leading term of the asymptotics of the $q$-hypergeometric solutions can be written as the equivariant Gamma class of the tangent bundle of $T^{*} \mathcal{F}_{\boldsymbol{\lambda}}$ multiplied by the exponentials of the equivariant first Chern classes of the associated vector bundles. That statement is analogous to the statement of the gamma conjecture by B. Dubrovin and by S. Galkin, V. Golyshev, and H. Iritani, see also the Gamma theorem for $\mathcal{F}_{\boldsymbol{\lambda}}$ in Appendix B.
\end{abstract}

In memory of Victor Lomonosov (1946-2018)

\section{Contents}

1. Introduction 3

2. Dynamical and $q K Z$ equations $\quad 4$

2.1. Notations 4

2.2. Dynamical differential equations 4

2.3. Difference $q K Z$ equations 5

3. Weight functions 5

3.1. Weight functions $\check{W}_{I} \quad 5$

3.2. Weight functions $\check{W}_{\sigma, I} \quad 6$

3.3. Three-term relation 6

${ }^{\circ} E$-mail: vt@math.iupui.edu,vt@pdmi.ras.ru

${ }^{\star} E$-mail: anv@email.unc.edu, supported in part by NSF grants DMS-1362924, DMS-1665239 
3.4. Weight functions $W_{I}(\boldsymbol{t} ; \boldsymbol{z}) \quad 7$

3.5. Modification of the three-term relation $\quad 7$

$\begin{array}{ll}3.6 . & \text { Shuffle properties }\end{array}$

3.7. Factorization $\quad 8$

3.8. Useful identities $\quad 8$

4. Master function and discrete differentials 9

4.1. Master function $\quad 9$

4.2. Definition of discrete differentials $\quad 10$

4.3. Special discrete differentials $\quad 10$

4.4. First key formula 11

4.5. Second key formula 12

5. Proof of Theorem $4.3 \quad 12$

6. Proof of Theorem $4.4 \quad 14$

6.1. Proof of Theorem 4.4 for $N=2, \boldsymbol{\lambda}=(n, 0), I=(\{1, \ldots, n\}, \emptyset) \quad 14$

6.2. Proof of Theorem 4.4 for $N=2$ and $I=I^{\max } \quad 15$

6.3. Proof of Theorem 4.4 for $i=1$, arbitrary $N$, and $I=(\{1, \ldots, n\}, \emptyset, \ldots, \emptyset) \quad 16$

6.4. Proof of Theorem 4.4 for $i=1$, arbitrary $N$, and $I=I^{\max } 16$

6.5. Proof of Theorem 4.4 for $i>1$, arbitrary $N$, and $I=(\{1, \ldots, n\}, \emptyset, \ldots, \emptyset) \quad 17$

6.6. Proof of Theorem 4.4 for $i>1$, arbitrary $\lambda$, and $I=I^{\max } 17$

6.7. Modification of the three-term relation 20

6.8. The end of the proof of Theorem $4.4 \quad 21$

7. Corollary of Theorems 4.3 and $4.4 \quad 22$

8. Integral representations for solutions of dynamical equations 24

8.1. Formal integrals 24

8.2. Jackson integral 24

8.3. Solutions of dynamical equations 26

9. Equivariant quantum differential equations 27

9.1. Partial flag varieties $\quad 27$

9.2. Equivariant cohomology 27

9.3. Stable envelope map 28

9.4. $H_{T}^{*}\left(T^{*} \mathcal{F}_{\boldsymbol{\lambda}}\right)$-valued weight function $\quad 29$

9.5. Quantum multiplication by divisors on $H_{T}^{*}\left(T^{*} \mathcal{F}_{\boldsymbol{\lambda}}\right) \quad 30$

10. Quantum Pieri rules 33

10.1. Quantum equivariant cohomology algebra $\mathcal{H}_{T}^{\tilde{q}}\left(T^{*} \mathcal{F}_{\boldsymbol{\lambda}}\right) \quad 33$

10.2. Quantum equivariant Pieri rules 34

10.3. Bethe ansatz equations $\quad 35$

10.4. Proof of Theorem $10.2 \quad 35$

10.5. Limit $\tilde{q}_{i} / \tilde{q}_{i+1} \rightarrow 0, i=1, \ldots, N-1$, and CSM classes of Schubert cells 36

11. Solutions of quantum differential equations and equivariant $K$-theory 36

11.1. Solutions and equivariant $K$-theory 36

11.2. End of proof of Lemma 11.2 38

11.3. The homogeneous case $\boldsymbol{z}=0 \quad 39$

11.4. The limit $h \rightarrow \infty \quad 40$

Appendix A. Basics on Schubert polynomials 42

Appendix B. Leading Terms of Solutions and Gamma Conjecture 44

References $\quad 45$ 


\section{INTRODUCTION}

In [MO], D. Maulik and A. Okounkov develop a general theory connecting quantum groups and equivariant quantum cohomology of Nakajima quiver varieties, see [N1, N2]. In particular, in $[\mathrm{MO}]$ the operators of quantum multiplication by divisors are described. As it is well-known, these operators determine the equivariant quantum differential equations of a quiver variety. In this paper we apply this description to the cotangent bundles $T^{*} \mathcal{F}_{\boldsymbol{\lambda}}$ of the $\mathfrak{g l}_{n} \quad N$-step partial flag varieties and construct $q$-hypergeometric solutions of the associated equivariant quantum differential equations and $q K Z$ difference equations. The $q$-hypergeometric solutions are constructed in the form of Jackson integrals.

Studying solutions of the equivariant quantum differential equations may lead to better understanding Gromov-Witten invariants of the cotangent bundle, cf. Givental's study of the $J$-function in [Gi1, Gi2, Gi3].

The presentation of solutions of the equivariant quantum differential equations as $q$ hypergeometric integrals manifests a version of the Landau-Ginzburg mirror symmetry for the cotangent bundle.

In $[\mathrm{MO}]$ the equivariant quantum differential equations come together with a compatible system of difference equations called the $q K Z$ equations. In [GRTV, RTV1] the equivariant quantum differential equations and $q K Z$ difference equations were identified with the dynamical differential equations and $q K Z$ difference equations with values in the tensor product $\left(\mathbb{C}^{N}\right)^{\otimes n}$ of vector representations of $\mathfrak{g l}_{N}$. The $q$-hypergeometric solutions of the $\left(\mathbb{C}^{N}\right)^{\otimes n}$-valued $q K Z$ difference equations were constructed long time ago in [TV1], see also [TV2]-[TV4]. It was expected that those $q$-hypergeometric solutions are also solutions of the compatible dynamical differential equations. That fact is proved in this paper and is the first main result of the paper. The proof is based on some new rather nontrivial identities for the integrand of the Jackson integral. The integrand is the product of the scalar master function and a vector-valued function, whose coordinates are called weight functions. In [RTV1] it was shown that the weight functions are nothing else but the stable envelopes of $[\mathrm{MO}]$ for the cotangent bundle of the partial flag varieties. Our new identities can be interpreted as new identities for stable envelopes. We interpret these new identities as Pieri rules in quantum equivariant cohomology of the cotangent bundle of the partial flag variety. That is our second main result.

Our Gamma theorem for $T^{*} \mathcal{F}_{\boldsymbol{\lambda}}$ (Theorem B.1) says that the leading term of the asymptotics of the $q$-hypergeometric solutions for $T^{*} \mathcal{F}_{\boldsymbol{\lambda}}$ is the product of the equivariant gamma class of the tangent bundle of $T^{*} \mathcal{F}_{\boldsymbol{\lambda}}$ and the exponentials of the equivariant first Chern classes of the associated vector bundles. That statement is analogous to the statement of the gamma conjecture by B. Dubrovin and by S. Galkin, V. Golyshev, and H. Iritani, see Appendix B. See also the Gamma theorem for $\mathcal{F}_{\boldsymbol{\lambda}}$ (Theorem B.2).

The paper is organized as follows. In Section 2 we introduce the $\left(\mathbb{C}^{N}\right)^{\otimes n}$-valued dynamical and $q K Z$ equations. In Section 3 we define the weight functions and list their basic properties. In Section 4 we introduce the master function and describe the discrete differentials - the quantities with zero Jackson integrals. We also formulate there two key identities for the weight functions - Theorems 4.3 and 4.4. We prove Theorem 4.3 in Section 5 and Theorem 4.4 in Section 6. In Section 7, we summarize Theorems 4.3 and 4.4 as a statement about the integrand of the main Jackson integral. In Section 8 we construct integral representations 
for solutions of the $\left(\mathbb{C}^{N}\right)^{\otimes n}$-valued dynamical equations. In Section 9 we introduce the equivariant quantum differential equations and explain how their $q$-hypergeometric solutions are obtained from solutions of the $\left(\mathbb{C}^{N}\right)^{\otimes n}$-valued dynamical equations. In Section 10 we formulate and prove Pieri rules. In Section 11 we show that the space of solutions of the quantum differential equation can be identified with the vector space of the equivariant Ktheory algebra. We also discuss two limiting cases of the quantum differential equation. In Appendix A we discuss the basic properties of Schubert polynomials, and in Appendix B we formulate our Gamma theorems.

The authors thank G. Cotti, V. Golyshev, and R. Rimanyi for useful discussions. The second author thanks the Hausdorff Institute for Mathematics in Bonn for hospitality in March 2018, when the Gamma theorem was discovered. The second author also thanks the Max Planck Institute for Mathematics in Bonn for hospitality in May-June 2018.

\section{DynAmiCAL And $q K Z$ EQUATIONS}

2.1. Notations. Fix $N, n \in \mathbb{Z}_{>0}$ and $h, \kappa \in \mathbb{C}^{\times}$. Let $\boldsymbol{\lambda} \in \mathbb{Z}_{\geqslant 0}^{N},|\boldsymbol{\lambda}|=\lambda_{1}+\ldots+\lambda_{N}=n$. Let $I=\left(I_{1}, \ldots, I_{N}\right)$ be a partition of $\{1, \ldots, n\}$ into disjoint subsets $I_{1}, \ldots, I_{N}$. Denote $\mathcal{I}_{\boldsymbol{\lambda}}$ the set of all partitions $I$ with $\left|I_{j}\right|=\lambda_{j}, j=1, \ldots, N$.

Consider $\mathbb{C}^{N}$ with basis $v_{i}=\left(0, \ldots, 0,1_{i}, 0, \ldots, 0\right), i=1, \ldots, N$, and the tensor product $\left(\mathbb{C}^{N}\right)^{\otimes n}$ with basis

$$
v_{I}=v_{i_{1}} \otimes \cdots \otimes v_{i_{n}},
$$

where the index $I$ is a partition $\left(I_{1}, \ldots, I_{N}\right)$ of $\{1, \ldots, n\}$ into disjoint subsets $I_{1}, \ldots, I_{N}$ and $i_{j}=m$ if $j \in I_{m}$.

The space $\left(\mathbb{C}^{N}\right)^{\otimes n}$ is a module over the Lie algebra $\mathfrak{g l}_{N}$ with basis $e_{i, j}, i, j=1, \ldots, N$. The $\mathfrak{g l}_{N}$-module $\left(\mathbb{C}^{N}\right)^{\otimes n}$ has weight decomposition $\left(\mathbb{C}^{N}\right)^{\otimes n}=\sum_{|\boldsymbol{\lambda}|=n}\left(\mathbb{C}^{N}\right)_{\lambda}^{\otimes n}$, where $\left(\mathbb{C}^{N}\right)_{\lambda}^{\otimes n}$ is the subspace with basis $\left(v_{I}\right)_{I \in \mathcal{I}_{\boldsymbol{\lambda}}}$.

2.2. Dynamical differential equations. Define the linear operators $X_{1}, \ldots, X_{n}$ acting on $\left(\mathbb{C}^{N}\right)^{\otimes n}$-valued functions of $\boldsymbol{z}=\left(z_{1}, \ldots, z_{n}\right), \boldsymbol{q}=\left(q_{1}, \ldots, q_{N}\right)$ and called the dynamical Hamiltonians:

$$
\begin{aligned}
X_{i}(\boldsymbol{z} ; h ; \boldsymbol{q})=\sum_{a=1}^{n} z_{a} e_{i, i}^{(a)}-h\left(\frac{\tilde{e}_{i, i}\left(1-\tilde{e}_{i, i}\right)}{2}\right. & +\sum_{\substack{1 \leqslant a<b \leqslant n \\
\sum_{k=1}}}^{N} e_{i, k}^{(a)} e_{k, i}^{(b)}+ \\
& \left.+\sum_{\substack{j=1 \\
j \neq i}}^{N} \frac{q_{j}}{q_{i}-q_{j}}\left(\tilde{e}_{i, j} \tilde{e}_{j, i}-\tilde{e}_{i, i}\right)\right),
\end{aligned}
$$

where $\tilde{e}_{s, t}=\sum_{a=1}^{n} e_{s, t}^{(a)}$ and a superscript means that the corresponding operator acts on the corresponding tensor factor. The differential operators

$$
\nabla_{\boldsymbol{q}, \kappa, i}=\kappa q_{i} \frac{\partial}{\partial q_{i}}-X_{i}(\boldsymbol{z} ; h ; \boldsymbol{q}), \quad i=1, \ldots, N,
$$

preserve the weight decomposition of $\left(\mathbb{C}^{N}\right)^{\otimes n}$ and pairwise commute, see [TV2], also [GRTV, Section 3.4], [RTV1, Section 7.1], [MTV1]. The operators $\nabla_{\boldsymbol{q}, \kappa, i}$ define the $\left(\mathbb{C}^{N}\right)^{\otimes n}$-valued 
dynamical connection. The system of differential equations

$$
\kappa q_{i} \frac{\partial f}{\partial q_{i}}=X_{i}(\boldsymbol{z} ; h ; \boldsymbol{q}) f, \quad i=1, \ldots, N
$$

on a $\left(\mathbb{C}^{N}\right)^{\otimes n}$-valued function $f(\boldsymbol{z} ; h ; \boldsymbol{q})$ is called the dynamical equations.

2.3. Difference $\boldsymbol{q} \mathbf{K Z}$ equations. Define the $R$-matrices acting on $\left(\mathbb{C}^{N}\right)^{\otimes n}$,

$$
R^{(i, j)}(u)=\frac{u-h P^{(i, j)}}{u-h}, \quad i, j=1, \ldots, n, \quad i \neq j
$$

Define the $q K Z$ operators $K_{1}, \ldots, K_{n}$ acting on $\left(\mathbb{C}^{N}\right)^{\otimes n}$ :

$$
\begin{aligned}
K_{i}(\boldsymbol{z} ; h ; \boldsymbol{q} ; \kappa) & =R^{(i, i-1)}\left(z_{i}-z_{i-1}+\kappa\right) \ldots R^{(i, 1)}\left(z_{i}-z_{1}+\kappa\right) \times \\
& \times q_{1}^{e_{1,1}^{(i)}} \ldots q_{N}^{e_{N, N}^{(i)}} R^{(i, n)}\left(z_{i}-z_{n}\right) \ldots R^{(i, i+1)}\left(z_{i}-z_{i+1}\right) .
\end{aligned}
$$

The $q K Z$ operators preserve the weight decomposition of $\left(\mathbb{C}^{N}\right)^{\otimes n}$ and form a discrete flat connection,

$$
K_{i}\left(z_{1}, \ldots, z_{j}+\kappa, \ldots, z_{n} ; \boldsymbol{q} ; \kappa\right) K_{j}(\boldsymbol{z} ; h ; \boldsymbol{q} ; \kappa)=K_{j}\left(z_{1}, \ldots, z_{i}+\kappa, \ldots, z_{n} ; \boldsymbol{q} ; \kappa\right) K_{i}(\boldsymbol{z} ; h ; \boldsymbol{q} ; \kappa)
$$

for all $i, j$, see $[\mathrm{FR}]$. The system of difference equations with step $\kappa$,

$$
f\left(z_{1}, \ldots, z_{i}+\kappa, \ldots, z_{n} ; \boldsymbol{q}\right)=K_{i}(\boldsymbol{z} ; h ; \boldsymbol{q} ; \kappa) f\left(z_{1}, \ldots, z_{n} ; \boldsymbol{q}\right), \quad i=1, \ldots, N,
$$

on a $\left(\mathbb{C}^{N}\right)^{\otimes n}$-valued function $f(\boldsymbol{z}, \boldsymbol{q})$ is called the $q K Z$ equations.

Theorem 2.1 ([TV2]). The systems of dynamical and qKZ equations are compatible.

\section{Weight Functions}

3.1. Weight functions $\check{W}_{I}$. For $I \in \mathcal{I}_{\boldsymbol{\lambda}}$, we define the weight functions $\check{W}_{I}(\boldsymbol{t} ; \boldsymbol{z})$, cf. [TV1, TV4, RTV1]. The functions $\breve{W}_{I}(\boldsymbol{t} ; \boldsymbol{z})$ here coincide with the functions $W_{I}(\boldsymbol{t} ; \boldsymbol{z} ; h)$ defined in [RTV1, Section 3.1].

Recall $\boldsymbol{\lambda}=\left(\lambda_{1}, \ldots, \lambda_{N}\right)$. Denote $\lambda^{(i)}=\lambda_{1}+\ldots+\lambda_{i}, i=1, \ldots, N-1, \lambda^{(N)}=n$, and $\lambda^{\{1\}}=\sum_{i=1}^{N-1} \lambda^{(i)}=\sum_{i=1}^{N-1}(N-i) \lambda_{i}$. Recall $I=\left(I_{1}, \ldots, I_{N}\right)$. Set $\bigcup_{k=1}^{j} I_{k}=\left\{i_{1}^{(j)}<\ldots<\right.$ $\left.i_{\lambda^{(j)}}^{(j)}\right\}$. Consider the variables $h$ and $t_{a}^{(j)}, j=1, \ldots, N-1, a=1, \ldots, \lambda^{(j)}$. Set $t_{a}^{(N)}=z_{a}$, $a=1, \ldots, n$. Denote $\boldsymbol{t}^{(j)}=\left(t_{k}^{(j)}\right)_{k \leqslant \lambda^{(j)}}$ and $\boldsymbol{t}=\left(\boldsymbol{t}^{(1)}, \ldots, \boldsymbol{t}^{(N-1)}\right)$.

The weight functions are

$$
\begin{aligned}
& \check{W}_{I}(\boldsymbol{t} ; \boldsymbol{z})=(-h)^{\lambda^{\{1\}}} \operatorname{Sym}_{t_{1}^{(1)}, \ldots, t_{\lambda^{(1)}}^{(1)}} \ldots \operatorname{Sym}_{t_{1}^{(N-1)}, \ldots, t_{\lambda}^{(N-1)}\left(\check{U}_{I}(\boldsymbol{t} ; \boldsymbol{z}),\right.} \\
& \check{U}_{I}(\boldsymbol{t} ; \boldsymbol{z})=\prod_{j=1}^{N-1} \prod_{a=1}^{\lambda^{(j)}}\left(\prod_{\substack{c=1 \\
i_{c}^{(j+1)}<i_{a}^{(j)}}}^{\lambda^{(j+1)}}\left(t_{a}^{(j)}-t_{c}^{(j+1)}-h\right) \prod_{\substack{d=1 \\
i_{d}^{(j+1)}>i_{a}^{(j)}}}^{\lambda^{(j+1)}}\left(t_{a}^{(j)}-t_{d}^{(j+1)}\right) \prod_{b=a+1}^{\lambda^{(j)}} \frac{t_{a}^{(j)}-t_{b}^{(j)}-h}{t_{a}^{(j)}-t_{b}^{(j)}}\right) .
\end{aligned}
$$


In these formulas for a function $f\left(t_{1}, \ldots, t_{k}\right)$ of some variables, we denote

$$
\operatorname{Sym}_{t_{1}, \ldots, t_{k}} f\left(t_{1}, \ldots, t_{k}\right)=\sum_{\sigma \in S_{k}} f\left(t_{\sigma_{1}}, \ldots, t_{\sigma_{k}}\right) .
$$

Example. Let $N=2, n=2, \boldsymbol{\lambda}=(1,1), I=(\{1\},\{2\}), J=(\{2\},\{1\})$. Then

$$
\check{W}_{I}(\boldsymbol{t} ; \boldsymbol{z})=-h\left(t_{1}^{(1)}-z_{2}\right), \quad \check{W}_{J}(\boldsymbol{t} ; \boldsymbol{z})=-h\left(t_{1}^{(1)}-z_{1}-h\right) .
$$

Example. Let $N=2, n=3, \boldsymbol{\lambda}=(1,2), I=(\{2\},\{1,3\})$. Then

$$
\check{W}_{I}(\boldsymbol{t} ; \boldsymbol{z})=-h\left(t_{1}^{(1)}-z_{1}-h\right)\left(t_{1}^{(1)}-z_{3}\right) .
$$

Example. Let $N=2, n=3, \boldsymbol{\lambda}=(2,1), I=(\{1,3\},\{2\})$. Then

$$
\begin{aligned}
\check{W}_{I}(\boldsymbol{t} ; \boldsymbol{z})=(-h)^{2} & \left(\left(t_{1}^{(1)}-z_{2}\right)\left(t_{1}^{(1)}-z_{3}\right)\left(t_{2}^{(1)}-z_{1}-h\right)\left(t_{2}^{(1)}-z_{2}-h\right) \frac{t_{1}^{(1)}-t_{2}^{(1)}-h}{t_{1}^{(1)}-t_{2}^{(1)}}+\right. \\
& \left.+\left(t_{2}^{(1)}-z_{2}\right)\left(t_{2}^{(1)}-z_{3}\right)\left(t_{1}^{(1)}-z_{1}-h\right)\left(t_{1}^{(1)}-z_{2}-h\right) \frac{t_{2}^{(1)}-t_{1}^{(1)}-h}{t_{2}^{(1)}-t_{1}^{(1)}}\right) .
\end{aligned}
$$

For a subset $A=\left\{a_{1}, \ldots, a_{j}\right\} \subset\{1, \ldots, n\}$, denote $\boldsymbol{z}_{A}=\left(z_{a_{1}}, \ldots, z_{a_{j}}\right)$. For $I \in \mathcal{I}_{\boldsymbol{\lambda}}$, denote $\boldsymbol{z}_{I}=\left(\boldsymbol{z}_{I_{1}}, \ldots, \boldsymbol{z}_{I_{N}}\right)$. For $f\left(\boldsymbol{t}^{(1)}, \ldots, \boldsymbol{t}^{(N)}\right) \in \mathbb{C}\left[\boldsymbol{t}^{(1)}, \ldots, \boldsymbol{t}^{(N)}\right]^{S_{\lambda}(1) \times \ldots \times S_{\lambda}(N)}$, we define $f\left(\boldsymbol{z}_{I}\right)$ by substituting $\boldsymbol{t}^{(j)}=\left(\boldsymbol{z}_{I_{1}}, \ldots, \boldsymbol{z}_{I_{j}}\right), j=1, \ldots, N$.

3.2. Weight functions $\check{W}_{\sigma, I}$. For $\sigma \in S_{n}$ and $I \in \mathcal{I}_{\boldsymbol{\lambda}}$, we define

$$
\check{W}_{\sigma, I}(\boldsymbol{t} ; \boldsymbol{z})=\check{W}_{\sigma^{-1}(I)}\left(\boldsymbol{t} ; z_{\sigma(1)}, \ldots, z_{\sigma(n)}\right), \quad \check{U}_{\sigma, I}(\boldsymbol{t} ; \boldsymbol{z})=\check{U}_{\sigma^{-1}(I)}\left(\boldsymbol{t} ; z_{\sigma(1)}, \ldots, z_{\sigma(n)}\right),
$$

where $\sigma^{-1}(I)=\left(\sigma^{-1}\left(I_{1}\right), \ldots, \sigma^{-1}\left(I_{N}\right)\right)$.

Example. Let $N=2, n=2, \boldsymbol{\lambda}=(1,1), I=(\{1\},\{2\}), J=(\{2\},\{1\})$. Then

$$
\begin{array}{rlrl}
\check{W}_{\mathrm{id}, I}(\boldsymbol{t} ; \boldsymbol{z}) & =-h\left(t_{1}^{(1)}-z_{2}\right), & \check{W}_{\mathrm{id}, J}(\boldsymbol{t} ; \boldsymbol{z}) & =-h\left(t_{1}^{(1)}-z_{1}-h\right), \\
\check{W}_{s, I}(\boldsymbol{t} ; \boldsymbol{z}) & =-h\left(t_{1}^{(1)}-z_{2}-h\right), & \check{W}_{s, J}(\boldsymbol{t} ; \boldsymbol{z})=-h\left(t_{1}^{(1)}-z_{1}\right),
\end{array}
$$

where $s$ is the transposition.

\subsection{Three-term relation.}

Lemma 3.1 ([RTV1, Lemma 3.6]). For any $\sigma \in S_{n}, I \in \mathcal{I}_{\boldsymbol{\lambda}}, i=1, \ldots, n-1$, we have

$$
\check{W}_{\sigma s_{i, i+1}, I}=\frac{z_{\sigma(i)}-z_{\sigma(i+1)}}{z_{\sigma(i)}-z_{\sigma(i+1)}+h} \check{W}_{\sigma, I}+\frac{h}{z_{\sigma(i)}-z_{\sigma(i+1)}+h} \check{W}_{\sigma, s_{\sigma(i), \sigma(i+1)}(I)},
$$

where $s_{i, j} \in S_{n}$ is the transposition of $i$ and $j$. 
3.4. Weight functions $W_{I}(\boldsymbol{t} ; \boldsymbol{z})$. Let $\sigma_{0} \in S_{n}$ be the longest permutation, $\sigma_{0}(i)=n+1-i$, $i=1, \ldots, n$. For $I \in \mathcal{I}_{\boldsymbol{\lambda}}$, denote

$$
W_{I}(\boldsymbol{t} ; \boldsymbol{z})=(-h)^{-\lambda^{\{1\}}} \check{W}_{\sigma_{0}, I}(\boldsymbol{t} ; \boldsymbol{z}), \quad U_{I}(\boldsymbol{t} ; \boldsymbol{z})=\check{U}_{\sigma_{0}, I}(\boldsymbol{t} ; \boldsymbol{z}) .
$$

In other words, we have

$$
\begin{aligned}
& W_{I}(\boldsymbol{t} ; \boldsymbol{z})=\operatorname{Sym}_{t_{1}^{(1)}, \ldots, t_{\lambda(1)}^{(1)}} \ldots \operatorname{Sym}_{t_{1}^{(N-1)}, \ldots, t_{\lambda\left(\lambda^{(N-1)}\right.}^{(N-1)}} U_{I}(\boldsymbol{t} ; \boldsymbol{z}), \\
& U_{I}(\boldsymbol{t} ; \boldsymbol{z})= \\
& =\prod_{j=1}^{N-1} \prod_{a=1}^{\lambda^{(j)}}\left(\prod_{\substack{c=1 \\
i_{c}^{(j+1)}<i_{a}^{(j)}}}^{\lambda_{a}^{(j+1)}}\left(t_{a}^{(j)}-t_{c}^{(j+1)}\right) \prod_{\substack{d=1 \\
i_{d}^{(j+1)}>i_{a}^{(j)}}}^{\lambda_{a}^{(j+1)}}\left(t_{a}^{(j)}-t_{d}^{(j+1)}-h\right) \prod_{b=a+1}^{\lambda^{(j)}} \frac{t_{b}^{(j)}-t_{a}^{(j)}-h}{t_{b}^{(j)}-t_{a}^{(j)}}\right) .
\end{aligned}
$$

Example. Let $N=2, n=2, \boldsymbol{\lambda}=(1,1), I=(\{1\},\{2\}), J=(\{2\},\{1\})$. Then

$$
W_{I}(\boldsymbol{t} ; \boldsymbol{z})=t_{1}^{(1)}-z_{2}-h, \quad W_{J}(\boldsymbol{t} ; \boldsymbol{z})=t_{1}^{(1)}-z_{1} .
$$

3.5. Modification of the three-term relation. For a function $f\left(z_{1}, \ldots, z_{n}\right)$ and $i=1$, $\ldots, n-1$, define the operator $S_{i, i+1}$ by the formula

$$
S_{i, i+1} f\left(z_{1}, \ldots, z_{n}\right)=\frac{z_{i}-z_{i+1}-h}{z_{i}-z_{i+1}} f\left(z_{1}, \ldots, z_{i+1}, z_{i}, \ldots, z_{n}\right)+\frac{h}{z_{i}-z_{i+1}} f\left(z_{1}, \ldots, z_{n}\right) .
$$

Lemma 3.1 can be reformulated as follows.

Lemma 3.2. For any $I \in \mathcal{I}_{\boldsymbol{\lambda}}, i=1, \ldots, n-1$, we have

$$
\begin{aligned}
& W_{s_{i, i+1}(I)}(\boldsymbol{t} ; \boldsymbol{z})=\left(S_{i, i+1} W_{I}\right)(\boldsymbol{t} ; \boldsymbol{z}) \\
& =\frac{z_{i}-z_{i+1}-h}{z_{i}-z_{i+1}} W_{I}\left(\boldsymbol{t} ; z_{1}, \ldots, z_{i+1}, z_{i}, \ldots, z_{n}\right)+\frac{h}{z_{i}-z_{i+1}} W_{I}\left(\boldsymbol{t} ; z_{1}, \ldots, z_{n}\right) .
\end{aligned}
$$

3.6. Shuffle properties. Let $n, n_{1}, n_{2} \in \mathbb{Z}_{>0}, n=n_{1}+n_{2}$. Let $\boldsymbol{\lambda}^{1}, \boldsymbol{\lambda}^{2}, \boldsymbol{\lambda} \in \mathbb{Z}_{\geqslant 0}^{N},\left|\boldsymbol{\lambda}^{1}\right|=n_{1}$, $\left|\boldsymbol{\lambda}^{2}\right|=n_{2}, \boldsymbol{\lambda}=\boldsymbol{\lambda}^{1}+\boldsymbol{\lambda}^{2}$. Let $I^{1}=\left(I_{1}^{1}, \ldots, I_{N}^{1}\right)$ be a decomposition of the set $\left\{1, \ldots, n_{1}\right\}$ into subsets such that $\left|I_{j}^{1}\right|=\lambda_{j}^{1}$. Let $I^{2}=\left(I_{1}^{2}, \ldots, I_{N}^{2}\right)$ be a decomposition of the set $\left\{n_{1}+1, \ldots\right.$, $n\}$ into subsets such that $\left|I_{j}^{2}\right|=\lambda_{j}^{2}$. Define the decomposition $I=\left(I_{1}, \ldots, I_{N}\right)$ of the set $\{1$, $\ldots, n\}$ by the rule: $I_{j}=I_{j}^{1} \cup I_{j}^{2}$.

Consider the weight function $W_{I}$ of variables $\left(\boldsymbol{t}^{(1)}, \ldots, \boldsymbol{t}^{(N)}\right)$, where $\boldsymbol{t}^{(j)}=\left(t_{1}^{(j)}, \ldots, t_{\lambda^{(j)}}^{(j)}\right)$, $\lambda^{(j)}=\lambda_{1}+\ldots+\lambda_{j}, j=1, \ldots, N-1$, and $\boldsymbol{t}^{(N)}=\left(z_{1}, \ldots, z_{n}\right)$.

Consider the weight function $W_{I^{1}}$ of variables $\left(\tilde{\boldsymbol{t}}^{(1)}, \ldots, \tilde{\boldsymbol{t}}^{(N)}\right)$, where $\tilde{\boldsymbol{t}}^{(j)}=\left(t_{1}^{(j)}, \ldots, t_{\left(\boldsymbol{\lambda}^{1}\right)^{(1)}}^{(j)}\right)$, $\left(\boldsymbol{\lambda}^{1}\right)^{(j)}=\lambda_{1}^{1}+\ldots+\lambda_{j}^{1}, j=1, \ldots, N-1$, and $\tilde{\boldsymbol{t}}^{(N)}=\left(z_{1}, \ldots, z_{n_{1}}\right)$. Consider the weight function $W_{I^{2}}$ of variables $\check{\boldsymbol{t}}=\left(\check{\boldsymbol{t}}^{(1)}, \ldots, \check{\boldsymbol{t}}^{(N)}\right)$, where $\check{\boldsymbol{t}}^{(j)}=\left(t_{\left(\boldsymbol{\lambda}^{1}\right)^{(j)}+1}^{(j)}, \ldots, t_{\lambda^{(j)}}^{(j)}\right)$ for $j=1, \ldots, N-1$, and $\check{\boldsymbol{t}}^{(N)}=\left(z_{n_{1}+1}, \ldots, z_{n}\right)$. Denote $\left(\boldsymbol{\lambda}^{2}\right)^{(j)}=\lambda_{1}^{2}+\ldots+\lambda_{j}^{2}, j=1, \ldots, N-1$. 
Define the connection coefficient

$$
\begin{aligned}
C_{\boldsymbol{\lambda}^{1}, \boldsymbol{\lambda}^{2}}(\boldsymbol{t} ; \boldsymbol{z}) & =\prod_{j=1}^{N-1}\left[\left(\prod_{a=1}^{\left(\boldsymbol{\lambda}^{1}\right)^{(j)}} \prod_{b=\left(\boldsymbol{\lambda}^{1}\right)^{(j)}+1}^{\lambda^{(j)}} \frac{t_{b}^{(j)}-t_{a}^{(j)}-h}{t_{b}^{(j)}-t_{a}^{(j)}}\right) \times\right. \\
& \left.\times\left(\prod_{a=1}^{\left(\boldsymbol{\lambda}^{1}\right)^{(j)}} \prod_{c=\left(\boldsymbol{\lambda}^{1}\right)^{(j+1)}+1}^{\lambda^{(j+1)}}\left(t_{a}^{(j)}-t_{c}^{(j)}-h\right)\right)\left(\prod_{a=\left(\boldsymbol{\lambda}^{1}\right)^{(j)}+1}^{\lambda^{(j)}} \prod_{c=1}^{\left(\boldsymbol{\lambda}^{1}\right)^{(j+1)}}\left(t_{a}^{(j)}-t_{c}^{(j)}\right)\right)\right] .
\end{aligned}
$$

Lemma 3.3. We have

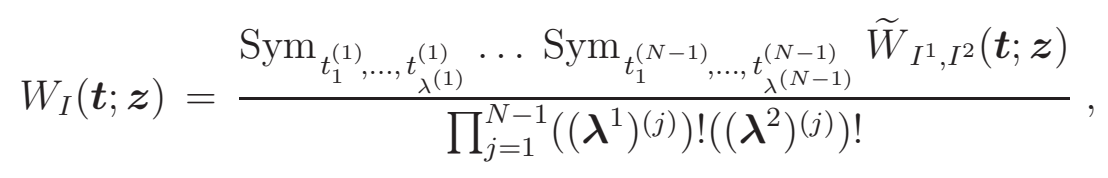

where

$$
\begin{array}{r}
\widetilde{W}_{I^{1}, I^{2}}(\boldsymbol{t} ; \boldsymbol{z})=C_{\boldsymbol{\lambda}^{1}, \boldsymbol{\lambda}^{2}}(\boldsymbol{t} ; \boldsymbol{z}) \\
W_{I^{1}}\left(\tilde{\boldsymbol{t}}^{(1)}, \ldots, \tilde{\boldsymbol{t}}^{(N-1)} ; z_{1}, \ldots, z_{n_{1}}\right) \\
\times W_{I^{2}}\left(\check{\boldsymbol{t}}^{(1)}, \ldots, \check{\boldsymbol{t}}^{(N-1)} ; z_{n_{1}+1}, \ldots, z_{n}\right) .
\end{array}
$$

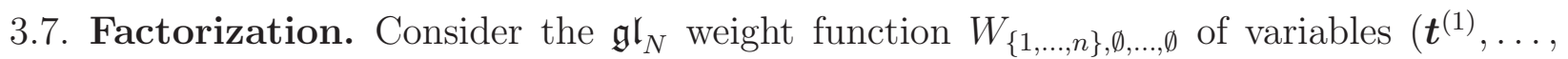
$\left.\boldsymbol{t}^{(N)}\right)$, where $\boldsymbol{t}^{(j)}=\left(t_{1}^{(j)}, \ldots, t_{n}^{(j)}\right)$ for $j=1, \ldots, N-1$, and $\boldsymbol{t}^{(N)}=\left(z_{1}, \ldots, z_{n}\right)$.

Lemma 3.4. We have

$$
W_{\{1, \ldots, n\}, \emptyset, \ldots, \emptyset}\left(\boldsymbol{t}^{(1)}, \ldots, \boldsymbol{t}^{(N-1)} ; \boldsymbol{t}^{(N)}\right)=\prod_{j=1}^{N-1} W_{\{1, \ldots, n\}, \emptyset}^{\mathfrak{g l}_{2}}\left(\boldsymbol{t}^{(j)} ; \boldsymbol{t}^{(j+1)}\right),
$$

where $W_{\{1, \ldots, n\}, \emptyset}^{\mathfrak{g l}_{2}}\left(\boldsymbol{t}^{(j)} ; \boldsymbol{t}^{(j+1)}\right)$ is the $\mathfrak{g l}_{2}$ weight function assigned to the partition of the set $\{1$, $\ldots, n\}$ into two subsets $\{1, \ldots, n\}$ and $\emptyset$.

Proof. The function $W_{\{1, \ldots, n\}, \emptyset}^{\mathfrak{g l}_{2}}\left(\boldsymbol{t}^{(j)} ; \boldsymbol{t}^{(j+1)}\right)$ is symmetric in variables $\left(t_{1}^{(j+1)}, \ldots, t_{n}^{(j+1)}\right)$ due to the $\mathfrak{g l}_{2}$ three-term relations of Lemma 3.1. That symmetry and formula (3.1) imply formula (3.11).

\subsection{Useful identities.}

Theorem 3.5. Given $k \in \mathbb{Z}_{\geqslant 0}$, consider variables $t_{1}^{(0)}, t_{1}^{(k+1)}$ and $t_{1}^{(i)}, t_{2}^{(i)}$ for $i=1, \ldots, k$. Set

$$
\begin{aligned}
& F=\left(t_{1}^{(0)}-t_{1}^{(1)}\right)\left(t_{1}^{(k)}-t_{1}^{(k+1)}-h\right)-\left(t_{1}^{(0)}-t_{2}^{(1)}-h\right)\left(t_{2}^{(k)}-t_{1}^{(k+1)}\right), \\
& G=\left(t_{1}^{(0)}-t_{2}^{(1)}-h\right)\left(t_{1}^{(k)}-t_{1}^{(k+1)}-h\right)-\left(t_{1}^{(0)}-t_{1}^{(1)}\right)\left(t_{2}^{(k)}-t_{1}^{(k+1)}\right),
\end{aligned}
$$

and

$$
H=\prod_{i=1}^{k-1}\left(\left(t_{1}^{(i)}-t_{2}^{(i+1)}-h\right)\left(t_{2}^{(i)}-t_{1}^{(i+1)}\right)\right) \prod_{i=1}^{k} \frac{t_{2}^{(i)}-t_{1}^{(i)}-h}{t_{2}^{(i)}-t_{1}^{(i)}} .
$$

Then

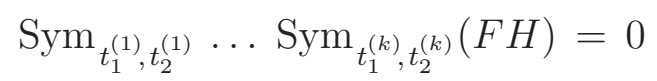


and

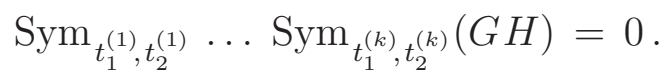

Proof. Formulae (3.12), (3.13) are equivalent to

Observe that

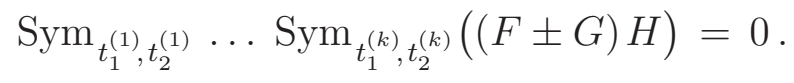

and

$$
\begin{gathered}
F+G=\left(2 t_{1}^{(0)}-t_{1}^{(1)}-t_{2}^{(1)}-h\right)\left(t_{1}^{(k)}-t_{2}^{(k)}-h\right), \\
F-G=\left(t_{1}^{(1)}-t_{2}^{(1)}-h\right)\left(t_{1}^{(k)}+t_{2}^{(k)}-2 t_{1}^{(k+1)}-h\right),
\end{gathered}
$$

$\operatorname{Sym}_{s_{1}, s_{2}}\left(\left(s_{1}-u_{2}-h\right)\left(s_{2}-u_{1}\right) \frac{s_{2}-s_{1}-h}{s_{2}-s_{1}}\right)=\operatorname{Sym}_{u_{1}, u_{2}}\left(\left(s_{1}-u_{2}-h\right)\left(s_{2}-u_{1}\right) \frac{u_{2}-u_{1}-h}{s_{2}-s_{1}}\right)$.

Hence the function

$$
W_{\{1,2\}, \emptyset}^{\mathfrak{g l}_{2}}\left(s_{1}, s_{2}, u_{1}, u_{2}\right)=\operatorname{Sym}_{s_{1}, s_{2}}\left(\left(s_{1}-u_{2}-h\right)\left(s_{2}-u_{1}\right) \frac{s_{2}-s_{1}-h}{s_{2}-s_{1}}\right)
$$

is symmetric both in $s_{1}, s_{2}$ and $u_{1}, u_{2}$. Therefore,

$$
\begin{aligned}
& \operatorname{Sym}_{t_{1}^{(1)}, t_{2}^{(1)}} \ldots \operatorname{Sym}_{t_{1}^{(k)}, t_{2}^{(k)}}((F+G) H)=\left(2 t_{1}^{(0)}-t_{1}^{(1)}-t_{2}^{(1)}-h\right) \times \\
& \quad \times \operatorname{Sym}_{t_{1}^{(k)}, t_{2}^{(k)}}\left(\left(t_{1}^{(k)}-t_{2}^{(k)}-h\right) \frac{t_{2}^{(k)}-t_{1}^{(k)}-h}{t_{2}^{(k)}-t_{1}^{(k)}}\right) \prod_{i=1}^{k-1} W_{\{1,2\}, \emptyset}^{\mathfrak{g l} \mathfrak{l}_{2}}\left(t_{1}^{(i)}, t_{2}^{(i)}, t_{1}^{(i+1)}, t_{2}^{(i+1)}\right)=0 .
\end{aligned}
$$

and

$$
\begin{aligned}
& \operatorname{Sym}_{t_{1}^{(1)}, t_{2}^{(1)}} \ldots \operatorname{Sym}_{t_{1}^{(k)}, t_{2}^{(k)}}((F-G) H)=\left(t_{1}^{(k)}+t_{2}^{(k)}-2 t_{1}^{(k+1)}-h\right) \times \\
& \times \operatorname{Sym}_{t_{1}^{(1)}, t_{2}^{(1)}}\left(\left(t_{1}^{(1)}-t_{2}^{(1)}-h\right) \frac{t_{2}^{(1)}-t_{1}^{(1)}-h}{t_{2}^{(1)}-t_{1}^{(1)}}\right) \prod_{i=1}^{k-1} W_{\{1,2\}, \emptyset}^{\mathfrak{g l}_{2}}\left(t_{1}^{(i)}, t_{2}^{(i)}, t_{1}^{(i+1)}, t_{2}^{(i+1)}\right)=0 .
\end{aligned}
$$

Theorem 3.5 is proved.

\section{MASTER FUnCTION AND DisCRETE DIFFERENTIALS}

4.1. Master function. Let $\phi(x)=\Gamma(x / \kappa) \Gamma((h-x) / \kappa)$. Define the master function:

$$
\begin{aligned}
& \Phi_{\boldsymbol{\lambda}}(\boldsymbol{t} ; \boldsymbol{z} ; h ; \boldsymbol{q})=\left(e^{\pi \sqrt{-1}\left(n-\lambda_{N}\right)} q_{N}\right)^{\sum_{a=1}^{n} z_{a} / \kappa} \prod_{i=1}^{N-1}\left(e^{\pi \sqrt{-1}\left(\lambda_{i+1}-\lambda_{i}\right)} \frac{q_{i}}{q_{i+1}}\right)^{\sum_{j=1}^{\lambda^{(i)}} t_{j}^{(i)} / \kappa} \times \\
& \times \prod_{i=1}^{N-1} \prod_{a=1}^{\lambda^{(i)}}\left(\prod_{\substack{\lambda^{(i)} \\
b \neq a}} \frac{1}{\left(t_{a}^{(i)}-t_{b}^{(i)}-h\right) \phi\left(t_{a}^{(i)}-t_{b}^{(i)}\right)} \prod_{c=1}^{\lambda^{(i+1)}} \phi\left(t_{a}^{(i)}-t_{c}^{(i+1)}\right)\right) .
\end{aligned}
$$

It is a symmetric function of variables in each of the groups $\boldsymbol{t}^{(i)}, i=1, \ldots, N-1$. 
4.2. Definition of discrete differentials. Consider the space $\mathcal{S}$ of functions of the form $\Phi_{\boldsymbol{\lambda}}(\boldsymbol{t} ; \boldsymbol{z} ; h ; \boldsymbol{q}) f(\boldsymbol{t} ; \boldsymbol{z} ; h ; \boldsymbol{q})$ where $f(\boldsymbol{t} ; \boldsymbol{z} ; h ; \boldsymbol{q})$ is a rational function. Consider the lattice $\kappa \mathbb{Z}^{\lambda^{\{1\}}}$ whose coordinates are labeled by variables $t_{j}^{(i)} \in \boldsymbol{t}$. The shifts $t_{j}^{(i)} \mapsto t_{j}^{(i)}+\kappa$ of any of the $\boldsymbol{t}$-variables preserve the space $\mathcal{S}$ and extend to an action of the lattice $\kappa \mathbb{Z}^{\lambda^{\{1\}}}$ on $\mathcal{S}$. A discrete differential is a finite sum of rational functions of the form

where $\boldsymbol{w} \in \kappa \mathbb{Z}^{\lambda^{\{1\}}}$.

$$
\frac{\Phi_{\boldsymbol{\lambda}}(\boldsymbol{t}+w ; \boldsymbol{z} ; h ; \boldsymbol{q})}{\Phi_{\boldsymbol{\lambda}}(\boldsymbol{t} ; \boldsymbol{z} ; h ; \boldsymbol{q})} f(\boldsymbol{t}+\boldsymbol{w} ; \boldsymbol{z} ; h ; \boldsymbol{q})-f(\boldsymbol{t} ; \boldsymbol{z} ; h ; \boldsymbol{q})
$$

4.3. Special discrete differentials. For integers $1 \leqslant \alpha<\beta \leqslant N$, split the variables $\boldsymbol{t}=$ $\left(\boldsymbol{t}^{(1)}, \ldots, \boldsymbol{t}^{(N-1)}\right), \boldsymbol{t}^{(i)}=\left(t_{1}^{(i)}, \ldots, t_{\lambda^{(i)}}^{(i)}\right)$, into two groups $\boldsymbol{t}^{\{\alpha, \beta\}}$ and $\boldsymbol{t}_{\{\alpha, \beta\}}$ as follows:

$$
\boldsymbol{t}^{\{\alpha, \beta\}}=\left(t_{\lambda^{(\alpha)}}^{(\alpha)}, t_{\lambda^{(\alpha+1)}}^{(\alpha+1)}, \ldots, t_{\lambda^{(\beta-1)}}^{(\beta-1)}\right)
$$

and $\boldsymbol{t}_{\{\alpha, \beta\}}=\left(\boldsymbol{t}_{\{\alpha, \beta\}}^{(1)}, \ldots, \boldsymbol{t}_{\{\alpha, \beta\}}^{(N-1)}\right)$, where $\boldsymbol{t}_{\{\alpha, \beta\}}^{(i)}=\left(t_{1}^{(i)}, \ldots, t_{\lambda^{(i)}-1}^{(i)}\right)$ if $\alpha \leqslant i<\beta$ and $\boldsymbol{t}_{\{\alpha, \beta\}}^{(i)}=$ $\boldsymbol{t}^{(i)}$, otherwise.

For a rational function $g$ of $\boldsymbol{t}_{\{\alpha, \beta\}}, \boldsymbol{z}, \boldsymbol{q}$, denote

$$
\begin{aligned}
d_{\boldsymbol{t}^{\{\alpha, \beta\}}} g & :=\frac{g\left(\boldsymbol{t}_{\{\alpha, \beta\}} ; \boldsymbol{z} ; h ; \boldsymbol{q}\right)}{q_{\alpha}-q_{\beta}}\left(q_{\beta}\left(t_{\lambda^{(\alpha-1)}}^{(\alpha-1)}-t_{\lambda^{(\alpha)}}^{(\alpha)}\right) \prod_{i=\alpha}^{\beta-1} \prod_{a=1}^{\lambda^{(i-1)}-1}\left(t_{a}^{(i-1)}-t_{\lambda^{(i)}}^{(i)}\right) \times\right. \\
& \times\left(t_{\lambda^{(\beta-1)}}^{(\beta-1)}-t_{\lambda^{(\beta)}}^{(\beta)}-h\right) \prod_{i=\alpha}^{\beta-1} \prod_{a=1}^{\lambda^{(i+1)}-1}\left(t_{\lambda^{(i)}}^{(i)}-t_{a}^{(i+1)}-h\right) \prod_{i=\alpha}^{\beta-1} \prod_{a=1}^{\lambda^{(i)}-1} \frac{t_{a}^{(i)}-t_{\lambda^{(i)}}^{(i)}-h}{t_{a}^{(i)}-t_{\lambda^{(i)}}^{(i)}}- \\
& -q_{\alpha}\left(t_{\lambda^{(\alpha-1)}}^{(\alpha-1)}-t_{\lambda^{(\alpha)}}^{(\alpha)}-h\right) \prod_{i=\alpha}^{\beta-1} \prod_{a=1}^{\lambda^{(i-1)}-1}\left(t_{a}^{(i-1)}-t_{\lambda^{(i)}}^{(i)}-h\right) \times \\
& \left.\times\left(t_{\lambda^{(\beta-1)}}^{(\beta-1)}-t_{\left.\lambda^{(\beta)}\right)}^{(\beta)}\right) \prod_{i=\alpha}^{\beta-1} \prod_{a=1}^{\lambda^{(i+1)}-1}\left(t_{\lambda^{(i)}}^{(i)}-t_{a}^{(i+1)}\right) \prod_{i=\alpha}^{\beta-1} \prod_{a=1}^{\lambda^{(i)}-1} \frac{t_{\lambda^{(i)}}^{(i)}-t_{a}^{(i)}-h}{t_{\lambda^{(i)}}^{(i)}-t_{a}^{(i)}}\right) .
\end{aligned}
$$

Lemma 4.1. The function $d_{\boldsymbol{t}^{\{\alpha, \beta\}}} \mathrm{g}$ is a discrete differential.

Proof. Formula (4.3) is an example of formula (4.2), where

$$
\begin{aligned}
f(\boldsymbol{t} ; \boldsymbol{z} ; h ; \boldsymbol{q}) & =g\left(\boldsymbol{t}_{\{\alpha, \beta\}} ; \boldsymbol{z} ; h ; \boldsymbol{q}\right)\left(t_{\lambda^{(\alpha-1)}}^{(\alpha-1)}-t_{\lambda^{(\alpha)}}^{(\alpha)}\right) \prod_{i=\alpha}^{\beta-1} \prod_{a=1}^{\lambda^{(i-1)}-1}\left(t_{a}^{(i-1)}-t_{\lambda^{(i)}}^{(i)}\right) \times \\
& \times\left(t_{\lambda^{(\beta-1)}}^{(\beta-1)}-t_{\lambda^{(\beta)}}^{(\beta)}-h\right) \prod_{i=\alpha}^{\beta-1} \prod_{a=1}^{\lambda^{(i+1)}-1}\left(t_{\lambda^{(i)}}^{(i)}-t_{a}^{(i+1)}-h\right) \prod_{i=\alpha}^{\beta-1} \prod_{a=1}^{\lambda^{(i)}-1} \frac{t_{a}^{(i)}-t_{\lambda^{(i)}}^{(i)}-h}{t_{a}^{(i)}-t_{\lambda^{(i)}}^{(i)}}
\end{aligned}
$$

and $\boldsymbol{w}$ has coordinates $t_{\lambda^{(\alpha)}}^{(\alpha)}, t_{\lambda^{(\alpha+1)}}^{(\alpha+1)}, \ldots, t_{\lambda^{(\beta-1)}}^{(\beta-1)}$ equal to $\kappa$ and other coordinates equal to zero. 
We rewrite $d_{\boldsymbol{t}\{\alpha, \beta\}} g$ as

$$
d_{\boldsymbol{t}^{\{\alpha, \beta\}}} g=\frac{q_{\beta}}{q_{\alpha}-q_{\beta}} \tilde{d}_{\boldsymbol{t}^{\{\alpha, \beta\}}} g-\check{d}_{\boldsymbol{t}^{\{\alpha, \beta\}}} g,
$$

where

$$
\begin{aligned}
& \tilde{d}_{\boldsymbol{t}^{\{\alpha, \beta\}}} g:=g\left(\boldsymbol{t}_{\{\alpha, \beta\}} ; \boldsymbol{z} ; h ; \boldsymbol{q}\right)\left(\left(t_{\lambda^{(\alpha-1)}}^{(\alpha-1)}-t_{\lambda^{(\alpha)}}^{(\alpha)}\right) \prod_{i=\alpha}^{\beta-1} \prod_{a=1}^{\lambda^{(i-1)}-1}\left(t_{a}^{(i-1)}-t_{\lambda^{(i)}}^{(i)}\right) \times\right. \\
& \times\left(t_{\lambda^{(\beta-1)}}^{(\beta-1)}-t_{\lambda^{(\beta)}}^{(\beta)}-h\right) \prod_{i=\alpha}^{\beta-1} \prod_{a=1}^{\lambda^{(i+1)}-1}\left(t_{\lambda^{(i)}}^{(i)}-t_{a}^{(i+1)}-h\right) \prod_{i=\alpha}^{\beta-1} \prod_{a=1}^{\lambda^{(i)}-1} \frac{t_{a}^{(i)}-t_{\lambda^{(i)}}^{(i)}-h}{t_{a}^{(i)}-t_{\lambda^{(i)}}^{(i)}}- \\
& \quad-\left(t_{\lambda^{(\alpha-1)}}^{(\alpha-1)}-t_{\lambda^{(\alpha)}}^{(\alpha)}-h\right) \prod_{i=\alpha}^{\beta-1} \prod_{a=1}^{\lambda^{(i-1)}-1}\left(t_{a}^{(i-1)}-t_{\lambda^{(i)}}^{(i)}-h\right) \times \\
&\left.\quad \times\left(t_{\lambda^{(\beta-1)}}^{(\beta-1)}-t_{\left.\lambda^{(\beta)}\right)}^{(\beta)}\right) \prod_{i=\alpha}^{\beta-1} \prod_{a=1}^{\lambda^{(i+1)}-1}\left(t_{\lambda^{(i)}}^{(i)}-t_{a}^{(i+1)}\right) \prod_{i=\alpha}^{\beta-1} \prod_{a=1}^{\lambda^{(i)}-1} \frac{t_{\lambda^{(i)}}^{(i)}-t_{a}^{(i)}-h}{t_{\lambda^{(i)}}^{(i)}-t_{a}^{(i)}}\right)
\end{aligned}
$$

and

$$
\begin{aligned}
\check{d}_{\boldsymbol{t}^{\{\alpha, \beta\}}} g & :=g\left(\boldsymbol{t}_{\{\alpha, \beta\}} ; \boldsymbol{z} ; h ; \boldsymbol{q}\right)\left(t_{\lambda^{(\alpha-1)}}^{(\alpha-1)}-t_{\lambda^{(\alpha)}}^{(\alpha)}-h\right) \prod_{i=\alpha}^{\beta-1} \prod_{a=1}^{\lambda^{(i-1)}-1}\left(t_{a}^{(i-1)}-t_{\lambda^{(i)}}^{(i)}-h\right) \times \\
& \times\left(t_{\lambda^{(\beta-1)}}^{(\beta-1)}-t_{\lambda^{(\beta)}}^{(\beta)}\right) \prod_{i=\alpha}^{\beta-1} \prod_{a=1}^{\lambda^{(i+1)}-1}\left(t_{\lambda^{(i)}}^{(i)}-t_{a}^{(i+1)}\right) \prod_{i=\alpha}^{\beta-1} \prod_{a=1}^{\lambda^{(i)}-1} \frac{t_{\lambda^{(i)}}^{(i)}-t_{a}^{(i)}-h}{t_{\lambda^{(i)}}^{(i)}-t_{a}^{(i)}} .
\end{aligned}
$$

Denote

$$
\begin{aligned}
& d_{\{\alpha, \beta\}} g:=\operatorname{Sym}_{t_{1}^{(1)}, \ldots, t_{\lambda(1)}^{(1)}} \ldots \operatorname{Sym}_{t_{1}^{(N-1)}, \ldots, t_{\lambda^{(N-1)}}^{(N-1)}} d_{\boldsymbol{t}^{\{\alpha, \beta\}}} g, \\
& \tilde{d}_{\{\alpha, \beta\}} g:=\operatorname{Sym}_{t_{1}^{(1)}, \ldots, t_{\lambda}^{(1)}} \ldots \operatorname{Sym}_{t_{1}^{(N-1)}, \ldots, t_{\lambda}^{(N-1)}} \tilde{d}_{\left.t^{(\alpha, \beta\}}, 1\right)} g, \\
& \check{d}_{\{\alpha, \beta\}} g:=\operatorname{Sym}_{t_{1}^{(1)}, \ldots, t_{\lambda}^{(1)}} \ldots \operatorname{Sym}_{t_{1}^{(N-1)}, \ldots, t_{\lambda(N-1)}^{(N-1)}} \check{d}_{\boldsymbol{t}\{\alpha, \beta\}} g .
\end{aligned}
$$

Then

$$
d_{\{\alpha, \beta\}} g=\frac{q_{\beta}}{q_{\alpha}-q_{\beta}} \tilde{d}_{\{\alpha, \beta\}} g-\check{d}_{\{\alpha, \beta\}} g .
$$

Corollary 4.2. The function $d_{\{\alpha, \beta\}} g$ is a discrete differential.

4.4. First key formula. Let $\boldsymbol{\lambda} \in \mathbb{Z}_{\geqslant 0}^{N},|\boldsymbol{\lambda}|=n$. For $\alpha, \beta=1, \ldots, N, \alpha \neq \beta$, denote

$$
\boldsymbol{\lambda}_{\alpha, \beta}=\left(\lambda_{1}, \ldots, \lambda_{\alpha}-1, \ldots, \lambda_{\beta}+1, \ldots, \lambda_{N}\right) .
$$

Notice that $\left|\boldsymbol{\lambda}_{\alpha, \beta}\right|=|\boldsymbol{\lambda}|$.

Let $I=\left(I_{1}, \ldots, I_{N}\right) \in \mathcal{I}_{\boldsymbol{\lambda}}, I_{k}=\left(\ell_{k, 1}, \ldots, \ell_{k, \lambda_{k}}\right), k=1, \ldots, N$. For $\alpha \neq \beta, a=1, \ldots$, $\lambda_{\alpha}, b=1, \ldots, \lambda_{\beta}$, denote

$$
(I)_{\beta, \alpha}^{b}=\left(I_{1}, \ldots, I_{\alpha} \cup\left\{\ell_{\beta, b}\right\}, \ldots, I_{\beta}-\left\{\ell_{\beta, b}\right\}, \ldots, I_{N}\right) \in \mathcal{I}_{\boldsymbol{\lambda}_{\alpha, \beta}} .
$$


For $J \in \mathcal{I}_{\boldsymbol{\lambda}_{\alpha, \beta}}$ and $b=1, \ldots, \lambda_{\beta}+1$, we have $(J)_{\beta, \alpha}^{b} \in \mathcal{I}_{\boldsymbol{\lambda}}$. The function $U_{J}$ defined by formula (3.5) is a function of variables $\boldsymbol{t}_{\alpha, \beta}, \boldsymbol{z}$.

Theorem 4.3. We have

$$
\left(\tilde{d}_{\{\alpha, \beta\}} U_{J}\right)(\boldsymbol{t} ; \boldsymbol{z})=-h \sum_{b=1}^{\lambda_{\beta}+1} W_{(J)_{\beta, \alpha}^{b}}(\boldsymbol{t} ; \boldsymbol{z}) .
$$

Theorem 4.3 is proved in Section 5.

4.5. Second key formula. Let $I=\left(I_{1}, \ldots, I_{N}\right) \in \mathcal{I}_{\boldsymbol{\lambda}}, I_{k}=\left(\ell_{k, 1}, \ldots, \ell_{k, \lambda_{k}}\right)$. For $k_{1}, k_{2}=$ $1, \ldots, N, k_{1} \neq k_{2}$, and $m_{1}=1, \ldots, \lambda_{k_{1}}, m_{2}=1, \ldots, \lambda_{k_{2}}$, define the element $I_{k_{1}, k_{2} ; m_{1}, m_{2}}=$ $\left(\tilde{I}_{1}, \ldots, \tilde{I}_{N}\right) \in \mathcal{I}_{\boldsymbol{\lambda}}$ such that $\tilde{I}_{k}=I_{k}$ if $k \neq k_{1}, k_{2}$, and

$$
\tilde{I}_{k_{1}}=I_{k_{1}} \cup\left\{\ell_{k_{2}, m_{2}}\right\}-\left\{\ell_{k_{1}, m_{1}}\right\}, \quad \tilde{I}_{k_{2}}=I_{k_{2}} \cup\left\{\ell_{k_{1}, m_{1}}\right\}-\left\{\ell_{k_{2}, m_{2}}\right\} .
$$

Theorem 4.4. For $I \in \mathcal{I}_{\boldsymbol{\lambda}}$ and $i=1, \ldots, N-1$, we have

$$
\begin{aligned}
\left(\sum_{j=1}^{\lambda^{(i)}} t_{j}^{(i)}\right. & \left.-\sum_{j=1}^{\lambda^{(i-1)}} t_{j}^{(i-1)}-\sum_{a \in I_{i}} z_{a}\right) W_{I} \\
= & h \sum_{j=1}^{i-1} \sum_{m_{1}=1}^{\lambda_{i}} \sum_{\substack{m_{2}=1 \\
\ell_{i, m_{1}}>\ell_{j, m_{2}}}}^{\lambda_{j}} W_{I_{i, j ; m_{1}, m_{2}}}-h \sum_{j=i+1}^{N} \sum_{m_{1}=1}^{\lambda_{i}} \sum_{\substack{m_{2}=1 \\
\ell_{i, m_{1}}<\ell_{j, m_{2}}}}^{\lambda_{j}} W_{I_{i, j ; m_{1}, m_{2}}}+ \\
& +\sum_{j=i+1}^{N} \sum_{a=1}^{\lambda_{i}} \check{d}_{\{i, j\}} U_{(I)_{i, j}^{a}}-\sum_{j=1}^{i-1} \sum_{a=1}^{\lambda_{j}} \check{d}_{\{j, i\}} U_{(I)_{j, i}^{a}} .
\end{aligned}
$$

Theorem 4.4 is proved in Section 6.

\section{Proof of Theorem 4.3}

For $n=1$, Theorem 4.3 is the following statement.

Lemma 5.1. Let $n=1$. For $1 \leqslant \gamma \leqslant n$, let $J^{\gamma}=\left(J_{1}, \ldots, J_{N}\right)$ be the decomposition of the one-element set $\{1\}$, such that $J_{\gamma}=\{1\}$ and $J_{j}=\emptyset$ for $j \neq \gamma$. Let $1 \leqslant \alpha<\beta \leqslant N$. Then

$$
\begin{array}{ll}
\tilde{d}_{\{\alpha, \beta\}} U_{J^{\gamma}}=-h W_{J^{\alpha}}, & \beta=\gamma, \\
\tilde{d}_{\{\alpha, \beta\}} U_{J^{\gamma}}=0, & \beta \neq \gamma .
\end{array}
$$

Proof. For any $\gamma$, we have $W_{J \gamma}=U_{J^{\gamma}}$, and $U_{J \gamma}$ is the function of $t_{1}^{(\gamma)}, \ldots, t_{1}^{(N-1)}, t_{1}^{(N)}=z_{1}$, which is identically equal to 1 , see $(3.5)$.

If $\beta=\gamma$, then

$$
\tilde{d}_{\{\alpha, \beta\}} U_{J^{\gamma}}=\tilde{d}_{\{\alpha, \gamma\}} U_{J^{\gamma}}=\left(t_{1}^{(\gamma-1)}-t_{1}^{(\gamma)}-h\right)-\left(t_{1}^{(\gamma-1)}-t_{1}^{(\gamma)}\right)=-h=-h W_{J^{\alpha}},
$$

which proves (5.1). 
The proof of (5.2) is by cases. If $\beta<\gamma$, then $\tilde{d}_{\{\alpha, \beta\}} U_{J \gamma}=(1-1) \cdot 1=0$. If $\gamma<\alpha<\beta$, then $\tilde{d}_{\{\alpha, \beta\}} U_{J \gamma}=0$ by identity (3.12). If $\alpha<\gamma<\beta$, then $\tilde{d}_{\{\alpha, \beta\}} U_{J \gamma}=0$ by identity (3.13). If $\alpha=\gamma<\beta$, then $\tilde{d}_{\{\alpha, \beta\}} U_{J \gamma}=0$ by the degeneration of identity (3.12) as $t_{1}^{(0)} \rightarrow \infty$.

For arbitrary $n$, Theorem 4.3 follows by induction on $n$ from the shuffle properties of weight functions in Lemma 3.3. To avoid writing numerous indices we illustrate the reasoning by an example.

Let $N=3, n=2, J=(\emptyset,\{1,2\}, \emptyset), \alpha=1, \beta=2$. Then formula (4.12) reads

$$
\tilde{d}_{\{1,2\}} U_{J}=-h W_{(\{1\},\{2\}, \emptyset)}-h W_{(\{2\},\{1\}, \emptyset)} .
$$

Indeed, we have

$$
\begin{array}{r}
\tilde{d}_{\{1,2\}} U_{J}=\operatorname{Sym}_{t_{1}^{(2)}, t_{2}^{(2)}}\left(\left(\left(t_{1}^{(1)}-t_{1}^{(2)}-h\right)\left(t_{1}^{(1)}-t_{2}^{(2)}-h\right)-\left(t_{1}^{(1)}-t_{1}^{(2)}\right)\left(t_{1}^{(1)}-t_{2}^{(2)}\right)\right) \times\right. \\
\left.\times\left(t_{1}^{(2)}-z_{2}-h\right)\left(t_{2}^{(2)}-z_{1}\right) \frac{t_{2}^{(2)}-t_{1}^{(2)}-h}{t_{2}^{(2)}-t_{1}^{(2)}}\right)= \\
=\operatorname{Sym}_{t_{1}^{(2)}, t_{2}^{(2)}}\left(\left(\left(t_{1}^{(1)}-t_{1}^{(2)}-h\right)\left(t_{1}^{(1)}-t_{2}^{(2)}-h\right)-\left(t_{1}^{(1)}-t_{1}^{(2)}\right)\left(t_{1}^{(1)}-t_{2}^{(2)}-h\right)+\right.\right. \\
\left.+\left(t_{1}^{(1)}-t_{1}^{(2)}\right)\left(t_{1}^{(1)}-t_{2}^{(2)}-h\right)-\left(t_{1}^{(1)}-t_{1}^{(2)}\right)\left(t_{1}^{(1)}-t_{2}^{(2)}\right)\right) \times \\
\left.\times\left(t_{1}^{(2)}-z_{2}-h\right)\left(t_{2}^{(2)}-z_{1}\right) \frac{t_{2}^{(2)}-t_{1}^{(2)}-h}{t_{2}^{(2)}-t_{1}^{(2)}}\right) .
\end{array}
$$

This is the sum of four terms. The first two are

$$
\begin{aligned}
& \operatorname{Sym}_{t_{1}^{(2)}, t_{2}^{(2)}}\left(\left(\left(t_{1}^{(1)}-t_{1}^{(2)}-h\right)\left(t_{1}^{(1)}-t_{2}^{(2)}-h\right)-\left(t_{1}^{(1)}-t_{1}^{(2)}\right)\left(t_{1}^{(1)}-t_{2}^{(2)}-h\right)\right) \times\right. \\
&\left.\times\left(t_{1}^{(2)}-z_{2}-h\right)\left(t_{2}^{(2)}-z_{1}\right) \frac{t_{2}^{(2)}-t_{1}^{(2)}-h}{t_{2}^{(2)}-t_{1}^{(2)}}\right)= \\
&=-h \operatorname{Sym}_{t_{1}^{(2)}, t_{2}^{(2)}}\left(\left(t_{1}^{(1)}-t_{2}^{(2)}-h\right)\left(t_{1}^{(2)}-z_{2}-h\right)\left(t_{2}^{(2)}-z_{1} \frac{t_{2}^{(2)}-t_{1}^{(2)}-h}{t_{2}^{(2)}-t_{1}^{(2)}}\right)=-h W_{\{1\},\{2\}, \emptyset},\right.
\end{aligned}
$$

the last two are

$$
\begin{gathered}
\operatorname{Sym}_{t_{1}^{(2)}, t_{2}^{(2)}}\left(\left(\left(t_{1}^{(1)}-t_{1}^{(2)}\right)\left(t_{1}^{(1)}-t_{2}^{(2)}-h\right)-\left(t_{1}^{(1)}-t_{1}^{(2)}\right)\left(t_{1}^{(1)}-t_{2}^{(2)}\right)\right) \times\right. \\
\left.\times\left(t_{1}^{(2)}-z_{2}-h\right)\left(t_{2}^{(2)}-z_{1}\right) \frac{t_{2}^{(2)}-t_{1}^{(2)}-h}{t_{2}^{(2)}-t_{1}^{(2)}}\right)= \\
=-h \operatorname{Sym}_{t_{1}^{(2)}, t_{2}^{(2)}}\left(\left(t_{1}^{(1)}-t_{2}^{(2)}\right)\left(t_{1}^{(2)}-z_{2}-h\right)\left(t_{2}^{(2)}-z_{1}\right) \frac{t_{2}^{(2)}-t_{1}^{(2)}-h}{t_{2}^{(2)}-t_{1}^{(2)}}\right)=-h W_{\{2\},\{1\}, \emptyset,}
\end{gathered}
$$

and we get (5.3).

The treatment of these four terms is an inductive step from $n=1$ to $n=2$. The analysis of the first two terms is the application of Theorem 4.3 for $n=1$ at the first point $z_{1}$. 
Namely, the factor $\left(\left(t_{1}^{(1)}-t_{1}^{(2)}-h\right)-\left(t_{1}^{(1)}-t_{2}^{(2)}\right)\right)$ corresponds to $\tilde{d}_{\{1,2\}}$ at $z_{1}$ and the product

$$
\left(t_{1}^{(1)}-t_{2}^{(2)}-h\right)\left(t_{1}^{(2)}-z_{2}-h\right)\left(t_{2}^{(2)}-z_{1}\right) \frac{t_{2}^{(2)}-t_{1}^{(2)}-h}{t_{2}^{(2)}-t_{1}^{(2)}}
$$

is the connection coefficient between $W_{\{1\}, \emptyset, \emptyset}$ sitting at $z_{1}$ and $W_{\emptyset,\{2\}, \emptyset}$ sitting at $z_{2}$, see Lemma 3.3. And the analysis of the last two terms is the application of Theorem 4.3 for $n=1$ at the second point $z_{2}$. Namely, the factor $\left(\left(t_{1}^{(1)}-t_{1}^{(2)}-h\right)-\left(t_{1}^{(1)}-t_{2}^{(2)}\right)\right)$ corresponds to $\tilde{d}_{\{1,2\}}$ at $z_{2}$ and the product

$$
\left(t_{1}^{(1)}-t_{2}^{(2)}\right)\left(t_{1}^{(2)}-z_{2}-h\right)\left(t_{2}^{(2)}-z_{1}\right) \frac{t_{2}^{(2)}-t_{1}^{(2)}-h}{t_{2}^{(2)}-t_{1}^{(2)}}
$$

is the connection coefficient between $W_{\{2\}, \emptyset, \emptyset}$ sitting at $z_{1}$ and $W_{\emptyset,\{1\}, \emptyset}$ sitting at $z_{2}$, see Lemma 3.3.

\section{Proof of Theorem 4.4}

6.1. Proof of Theorem 4.4 for $N=2, \boldsymbol{\lambda}=(n, 0), I=(\{1, \ldots, n\}, \emptyset)$.

Lemma 6.1. We have

$$
\sum_{l=1}^{n}\left(t_{l}^{(1)}-z_{l}\right) W_{\{1, \ldots, n\}, \emptyset}^{\mathfrak{g l} l_{2}}=\sum_{a=1}^{n} \check{d}_{\{1,2\}} U_{\{1, \ldots, a-1, a+1, \ldots, n\},\{a\}}^{\mathfrak{g l}_{2}} .
$$

Proof. We will prove formula (6.1) by induction on $n$. Denote

$$
\begin{gathered}
\boldsymbol{t}^{\prime}=\left(t_{1}^{(1)}, \ldots, t_{n-1}^{(1)}\right), \quad \boldsymbol{t}^{\prime \prime}=\left(t_{1}^{(1)}, \ldots, t_{n-2}^{(1)}\right), \quad \boldsymbol{z}^{\prime}=\left(z_{1}, \ldots, z_{n-1}\right), \\
A_{n}\left(\boldsymbol{t}, \boldsymbol{z}^{\prime}\right)=\prod_{b=1}^{n-1}\left(\left(t_{n}^{(1)}-z_{b}\right) \frac{t_{n}^{(1)}-t_{b}^{(1)}-h}{t_{n}^{(1)}-t_{b}^{(1)}}\right), \quad B\left(\boldsymbol{t}^{\prime \prime}, z\right)=\prod_{a=1}^{n-2}\left(t_{a}^{(1)}-z-h\right) .
\end{gathered}
$$

By formulae (4.5), (4.8), equality (6.1) reads as follows:

$$
\begin{aligned}
\operatorname{Sym}_{t_{1}^{(1)}, \ldots, t_{n}^{(1)}} \sum_{a=1}^{n} & \left(\left(t_{a}^{(1)}-z_{a}\right) U_{\{1, \ldots, n\}, \emptyset}^{\mathfrak{g l}_{2}}(\boldsymbol{t}, \boldsymbol{z})-\right. \\
& \left.-\left(t_{n}^{(1)}-z_{n}\right) A_{n}\left(\boldsymbol{t}, \boldsymbol{z}^{\prime}\right) U_{\{1, \ldots, a-1, a+1, \ldots, n\},\{a\}}^{\mathfrak{g l}_{2}}\left(\boldsymbol{t}^{\prime}, \boldsymbol{z}\right)\right)=0 .
\end{aligned}
$$

For $n=1$, formula (6.2) is clearly true. For the induction step, we explore formula (3.6). It implies that the summation term with $a=n$ in formula (6.2) vanishes,

$$
U_{\{1, \ldots, n\}, \emptyset}^{\mathfrak{g l} l_{2}}(\boldsymbol{t}, \boldsymbol{z})=A_{n}(\boldsymbol{t}, \boldsymbol{z}) U_{\{1, \ldots, n-1\}, \emptyset}^{\mathfrak{g l}_{2}}\left(\boldsymbol{t}^{\prime}, \boldsymbol{z}^{\prime}\right) B\left(\boldsymbol{t}^{\prime \prime}, z_{n}\right)\left(t_{n-1}^{(1)}-z_{n}-h\right),
$$

and for $a<n$,

$$
\begin{aligned}
& U_{\{1, \ldots, a-1, a+1, \ldots, n\},\{a\}}^{\mathfrak{g l}_{2}}\left(\boldsymbol{t}^{\prime}, \boldsymbol{z}\right)= \\
& =\left(t_{n-1}^{(1)}-z_{n-1}\right) A_{n-1}\left(\boldsymbol{t}^{\prime}, \boldsymbol{z}^{\prime}\right) U_{\{1, \ldots, a-1, a+1, \ldots, n-1\},\{a\}}^{\mathfrak{g l}_{2}}\left(\boldsymbol{t}^{\prime \prime}, \boldsymbol{z}^{\prime}\right) B\left(\boldsymbol{t}^{\prime \prime}, z_{n}\right) .
\end{aligned}
$$


The last formula and the identity

$$
\operatorname{Sym}_{t_{n-1}^{(n)}, t_{n}^{(n)}}\left(t_{n}^{(n)}-z_{n}\right) \frac{t_{n}^{(n)}-t_{n-1}^{(n)}-h}{t_{n}^{(n)}-t_{n-1}^{(n)}}=\operatorname{Sym}_{t_{n-1}^{(n)}, t_{n}^{(n)}}\left(t_{n-1}^{(n)}-z_{n}-h\right) \frac{t_{n}^{(n)}-t_{n-1}^{(n)}-h}{t_{n}^{(n)}-t_{n-1}^{(n)}},
$$

yield

$$
\begin{aligned}
\operatorname{Sym}_{t_{1}^{(1)}, \ldots, t_{n}^{(1)}}\left(t_{n}^{(1)}-z_{n}\right) A_{n}\left(\boldsymbol{t}, \boldsymbol{z}^{\prime}\right) U_{\{1, \ldots, a-1, a+1, \ldots, n\},\{a\}}^{\mathfrak{g l}_{2}}\left(\boldsymbol{t}^{\prime}, \boldsymbol{z}\right)= \\
=\operatorname{Sym}_{t_{1}^{(1)}, \ldots, t_{n}^{(1)}}\left(t_{n-1}^{(1)}-z_{n-1}\right) A_{n}\left(\boldsymbol{t}, \boldsymbol{z}^{\prime}\right) A_{n-1}\left(\boldsymbol{t}^{\prime}, \boldsymbol{z}^{\prime}\right) \times \\
\times U_{\{1, \ldots, a-1, a+1, \ldots, n-1\},\{a\}}^{\mathfrak{g l}_{2}}\left(\boldsymbol{t}^{\prime \prime}, \boldsymbol{z}^{\prime}\right) B\left(\boldsymbol{t}^{\prime \prime}, z_{n}\right)\left(t_{n-1}^{(1)}-z_{n}-h\right) .
\end{aligned}
$$

Summarizing all observations, we see that formula (6.2) follows from the equality

$$
\begin{aligned}
\operatorname{Sym}_{t_{1}^{(1)}, \ldots, t_{n-1}^{(1)}} & A_{n}\left(\boldsymbol{t}, \boldsymbol{z}^{\prime}\right) B\left(\boldsymbol{t}^{\prime \prime}, z_{n}\right)\left(t_{n-1}^{(1)}-z_{n}-h\right) \times \\
\times & \quad \sum_{a=1}^{n-1}\left(\left(t_{a}^{(1)}-z_{a}\right) U_{\{1, \ldots, n-1\}, \emptyset}^{\mathfrak{g l}_{2}}\left(\boldsymbol{t}^{\prime}, \boldsymbol{z}^{\prime}\right)-\right. \\
& \left.\quad-\left(t_{n-1}^{(1)}-z_{n-1}\right) A_{n-1}\left(\boldsymbol{t}^{\prime}, \boldsymbol{z}^{\prime}\right) U_{\{1, \ldots, a-1, a+1, \ldots, n-1\},\{a\}}^{\mathfrak{g l}_{2}}\left(\boldsymbol{t}^{\prime \prime}, \boldsymbol{z}^{\prime}\right)\right)=0,
\end{aligned}
$$

with $t_{n}^{(1)}$ not involved in the symmetrization. Since the product

$$
A_{n}\left(\boldsymbol{t}, \boldsymbol{z}^{\prime}\right) B\left(\boldsymbol{t}^{\prime \prime}, z_{n}\right)\left(t_{n-1}^{(1)}-z_{n}-h\right)
$$

is symmetric in $t_{1}^{(1)}, \ldots, t_{n-1}^{(1)}$, formula (6.6) follows from the induction assumption

$$
\begin{aligned}
\operatorname{Sym}_{t_{1}^{(1)}, \ldots, t_{n-1}(1)} \sum_{a=1}^{n-1} & \left(\left(t_{a}^{(1)}-z_{a}\right) U_{\{1, \ldots, n-1\}, \emptyset}^{\mathfrak{g l}_{2}}\left(\boldsymbol{t}^{\prime}, \boldsymbol{z}^{\prime}\right)-\right. \\
& \left.-\left(t_{n-1}^{(1)}-z_{n-1}\right) A_{n-1}\left(\boldsymbol{t}^{\prime}, \boldsymbol{z}^{\prime}\right) U_{\{1, \ldots, a-1, a+1, \ldots, n-1\},\{a\}}^{\mathfrak{g l}_{2}}\left(\boldsymbol{t}^{\prime \prime}, \boldsymbol{z}^{\prime}\right)\right)=0 .
\end{aligned}
$$

Lemma 6.1 is proved.

6.2. Proof of Theorem 4.4 for $N=2$ and $I=I^{\max }$. For $N=2, \boldsymbol{\lambda}=(k, n-k)$, we denote $I^{\max }=(\{n-k+1, \ldots, n\},\{1, \ldots, n-k\})$. Then formula (4.14) becomes formula (6.8) below.

Lemma 6.2. We have

$$
\begin{aligned}
& \sum_{l=1}^{k}\left(t_{l}^{(1)}-z_{n-k+l}\right) W_{\{n-k+1, \ldots, n\},\{1, \ldots, n-k\}}^{\mathfrak{g l}_{2}} \\
& \quad=\sum_{a=n-k+1}^{n} \check{d}_{\{1,2\}} U_{\{n-k+1, \ldots, a-1, a+1, \ldots, n\},\{1, \ldots, n-k, a\}}^{\mathfrak{g l}_{2}}
\end{aligned}
$$

Proof. Dividing both sides of the equation by $\prod_{i=1}^{k} \prod_{a=1}^{n-k}\left(t_{l}^{(1)}-z_{a}\right)$, turns formula (6.8) into formula (6.1). 
6.3. Proof of Theorem 4.4 for $i=1$, arbitrary $N$, and $I=(\{1, \ldots, n\}, \emptyset, \ldots, \emptyset)$.

Proposition 6.3. For $I=(\{1, \ldots, n\}, \emptyset, \ldots, \emptyset)$, we have

$$
\left(\sum_{l=1}^{n} t_{l}^{(1)}-\sum_{a=1}^{n} z_{a}\right) W_{I}=\sum_{j=2}^{n} \sum_{a=1}^{n} \check{d}_{\{1, j\}} U_{(I)_{1, j}^{\prime a}} .
$$

Proof. Formula (6.9) is equivalent to the formula

$$
\sum_{j=2}^{n} \sum_{l=1}^{n}\left(t_{l}^{(j-1)}-t_{l}^{(j)}\right) W_{I}=\sum_{j=2}^{n} \sum_{a=1}^{n} \check{d}_{\{1, j\}} U_{(I)_{1, j}^{\prime a}}
$$

which follows from the next lemma.

Lemma 6.4. For $j=2, \ldots, N$ we have

$$
\sum_{l=1}^{n}\left(t_{l}^{(j-1)}-t_{l}^{(j)}\right) W_{I}=\sum_{a=1}^{n} \check{d}_{\{1, j\}} U_{(I)_{1, j}^{\prime a}}
$$

Proof. By Lemma 3.4, the left-hand side of (6.10) equals

$$
\left(\sum_{l=1}^{n}\left(t_{l}^{(j-1)}-t_{l}^{(j)}\right) W_{\{1, \ldots, n\}, \emptyset}^{\mathfrak{g l} \mathfrak{l}_{2}}\left(\boldsymbol{t}^{(j-1)} ; \boldsymbol{t}^{(j)}\right)\right) \prod_{\substack{i=2 \\ i \neq j}}^{N} W_{\{1, \ldots, n\}, \emptyset}^{\mathfrak{g l} l_{2}}\left(\boldsymbol{t}^{(i-1)} ; \boldsymbol{t}^{(i)}\right) .
$$

It is easy to see that the right hand side equals

$$
\left(\sum_{a=1}^{n} \check{d}_{\{j-1, j\}} U_{(\{1, \ldots, a-1, a+1, \ldots, n\},\{a\})}\left(\boldsymbol{t}^{(j-1)} ; \boldsymbol{t}^{(j)}\right)\right) \prod_{\substack{i=2 \\ i \neq j}}^{N} W_{\{1, \ldots, n\}, \emptyset}^{\mathfrak{g l} l_{2}}\left(\boldsymbol{t}^{(i-1)} ; \boldsymbol{t}^{(i)}\right) .
$$

Hence, Lemma 6.4 follows from formula (6.1).

Proposition 6.3 is proved.

6.4. Proof of Theorem 4.4 for $i=1$, arbitrary $N$, and $I=I^{\max }$. For $\boldsymbol{\lambda}=\left(\lambda_{1}, \ldots\right.$, $\left.\lambda_{N}\right)$, we denote $I^{\max }=\left(\left\{n-\lambda_{1}+1, \ldots, n\right\}, \ldots,\left\{1, \ldots, \lambda_{N}\right\}\right)$. Then formula (4.14) takes the form

$$
\sum_{j=2}^{N} \sum_{l=1}^{\lambda_{1}}\left(t_{l+\lambda^{(j-1)}-\lambda_{1}}^{(j-1)}-t_{l+\lambda^{(j)}-\lambda_{1}}^{(j)}\right) W_{I^{\max }}=\sum_{j=2}^{N} \sum_{a=1}^{\lambda_{1}} \check{d}_{\{1, j\}} W_{\left(I^{\max }\right)_{1, j}^{\prime a}} .
$$

The following lemma implies formula (6.13).

Lemma 6.5. For $j=2, \ldots, N$, we have

$$
\sum_{l=1}^{\lambda_{1}}\left(t_{l+\lambda^{(j-1)}-\lambda_{1}}^{(j-1)}-t_{l+\lambda^{(j)}-\lambda_{1}}^{(j)}\right) W_{I^{\max }}=\sum_{a=1}^{\lambda_{1}} \check{d}_{\{1, j\}} W_{\left(I^{\max }\right)_{1, j}^{\prime a}} .
$$


Proof. The left-hand side of formula (6.14) equals

$$
\begin{aligned}
& \operatorname{Sym}_{t_{1}^{(1)}, \ldots, t_{\lambda(1)}^{(1)}} \ldots \operatorname{Sym}_{t_{1}^{(N-1)}, \ldots, t_{\lambda(N-1)}^{(N-1)}}\left(\sum_{l=1}^{\lambda_{1}}\left(t_{l+\lambda^{(j-1)}-\lambda_{1}}^{(j-1)}-t_{l+\lambda^{(j)}-\lambda_{1}}^{(j)}\right)\right.
\end{aligned}
$$

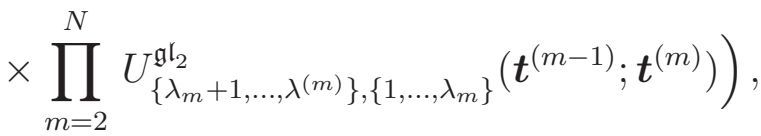

while the right-hand side of (6.14) equals by definition

$$
\begin{array}{r}
\operatorname{Sym}_{t_{1}^{(1)}, \ldots, t_{\lambda(1)}^{(1)} \ldots} \operatorname{Sym}_{t_{1}^{(N-1)}, \ldots, t_{\lambda^{(N-1)}}^{(N-1)}}\left(\prod_{l=1}^{\lambda^{(j)}}\left(t_{\lambda^{(j-1)}}^{(j-1)}-t_{l}^{(j)}\right) \prod_{l=1}^{\lambda^{(j-1)}-1} \frac{t_{\lambda^{(j-1)}}^{(j-1)}-t_{l}^{(j-1)}-h}{t_{\lambda^{(j-1)}}^{(j-1)}-t_{l}^{(j-1)}}\right. \\
\times \sum_{b=1+\lambda^{(j)}-\lambda_{1}}^{\lambda^{(j)}} U_{\left\{\lambda_{j}+1, \ldots, \lambda^{(j)}-\lambda_{1}, \ldots, b-1, b+1, \ldots, \lambda^{(j)}\right\},\left\{1, \ldots, \lambda_{j}, b\right\}}^{\mathfrak{g l}_{2}}\left(\boldsymbol{t}^{(j-1)} \backslash\left\{t_{\lambda^{(j-1)}}^{(j-1)}\right\}, \boldsymbol{t}^{(j)}\right) \\
\left.\times \prod_{\substack{m=2 \\
m \neq j}}^{N} U_{\left\{\lambda_{m}+1, \ldots, \lambda^{(m)}\right\},\left\{1, \ldots, \lambda_{m}\right\}}^{\mathfrak{g l}_{2}}\left(\boldsymbol{t}^{(m-1)}, \boldsymbol{t}^{(m)}\right)\right) .
\end{array}
$$

The equality of (6.15) and (6.16) follows from the following case of formula (6.1):

$$
\begin{aligned}
& \sum_{l=1}^{\lambda_{1}}\left(t_{l+\lambda^{(j-1)}-\lambda_{1}}^{(j-1)}-t_{l+\lambda^{(j)}-\lambda_{1}}^{(j)}\right) W_{\left\{1, \ldots, \lambda_{1}\right\}, \emptyset}^{\mathfrak{g l}_{2}}\left(t_{1+\lambda^{(j-1)}-\lambda_{1}}^{(j-1)}, \ldots, t_{\lambda^{(j-1)}}^{(j-1)} ; t_{1+\lambda^{(j)}-\lambda_{1}}^{(j)}, \ldots, t_{\lambda^{(j)}}^{(j)}\right) \\
& \quad=\sum_{a=1}^{\lambda_{1}} \check{d}_{\{1,2\}} U_{\left\{1, \ldots, a-1, a+1, \ldots, \lambda_{1}\right\},\{a\}}^{\mathfrak{g l}_{2}}\left(t_{1+\lambda^{(j-1)}-\lambda_{1}}^{(j-1)}, \ldots, t_{\lambda^{(j-1)}-1}^{(j-1)} ; t_{1+\lambda^{(j)}-\lambda_{1}}^{(j)}, \ldots, t_{\lambda^{(j)}}^{(j)}\right) .
\end{aligned}
$$

6.5. Proof of Theorem 4.4 for $i>1$, arbitrary $N$, and $I=(\{1, \ldots, n\}, \emptyset, \ldots, \emptyset)$. For $i=2, \ldots, N-1$, and $I=(\{1, \ldots, n\}, \emptyset, \ldots, \emptyset)$, Theorem 4.4 says that

$$
\sum_{l=1}^{n}\left(t_{l}^{(i)}-t_{l}^{(i-1)}\right) W_{I}=-\sum_{a=1}^{n} \check{d}_{\{1, i\}} U_{(I)_{1, j}^{\prime a}},
$$

which is formula (6.10).

6.6. Proof of Theorem $\mathbf{4 . 4}$ for $i>1$, arbitrary $\lambda$, and $I=I^{\text {max }}$. To prove this case of Theorem 4.4, we introduce a partition $I^{\max , j}=\left(I_{1}^{\max , j}, \ldots, I_{j}^{\max , j}\right)$ of the set $\left(1, \ldots, \lambda^{(j)}\right)$ by the rule

$$
I_{a}^{\max , j}=\left\{i \mid \lambda^{(j)}-\lambda^{(a)}<i \leqslant \lambda^{(j)}-\lambda^{(a-1)}\right\},
$$

so that $\left|I_{a}^{\max , j}\right|=\lambda_{a}$. For example, $I^{\max , N}=I^{\max }$. 
Formula (4.14) for $I=I^{\max }$ can be written as

$$
\begin{aligned}
& \sum_{l=1}^{j-1}\left(\left(\sum_{i \in I_{l}^{\max , j}} t_{i}^{(j)}-\sum_{i \in I_{l}^{\max , j-1}} t_{i}^{(j-1)}\right) W_{I^{\max }}+\sum_{a=1}^{\lambda_{l}} \check{d}_{\{l, j\}} U_{\left(I^{\max )_{l, j}^{\prime}}\right.}\right) \\
+ & \sum_{l=j+1}^{N}\left(\left(\sum_{i \in I_{j}^{\max , l-1}} t_{j}^{(l-1)}-\sum_{j \in I_{i}^{\max , l}} t_{i}^{(l)}\right) W_{I^{\max }}-\sum_{a=1}^{\lambda_{l}} \check{d}_{\{j, l\}} U_{\left(I^{\max }\right)_{j, l}^{\prime a}}\right)=0 .
\end{aligned}
$$

Formula (6.18) follows from the next Proposition.

Proposition 6.6. For $i=1,2, \ldots, N-1$, and $j=i, i+1, \ldots, N-1$, we have

$$
\left(\sum_{l \in I_{i}^{\max , j}} t_{l}^{(j)}-\sum_{l \in I_{i}^{\max , j}} t_{l}^{(j+1)}\right) W_{I^{\max }}=\sum_{a=1}^{\lambda_{i}} \check{d}_{\{i, j+1\}} U_{\left(I^{\max }\right)_{i, j+1}^{\prime a}} .
$$

Proof. For $i=1$, formula (6.19) follows from Lemma 6.5. For $i>1$, we prove formula (6.19) by induction on $\lambda^{(i-1)}$, see Lemmas 6.7 and 6.8 below. If $\lambda^{(i-1)}=0$, that is, $\lambda_{j}=0$ for all $j=1, \ldots, i-1$, formula (6.19) follows from Lemma 6.5 by renaming variables.

We will indicate explicitly the dependence of the partitions $I^{\max }, I^{\max , l}$ on $\boldsymbol{\lambda}$ :

$$
I_{\boldsymbol{\lambda}}^{\max }=\left(I_{\boldsymbol{\lambda}, 1}^{\max }, \ldots, I_{\boldsymbol{\lambda}, N}^{\max }\right), \quad I_{\boldsymbol{\lambda}}^{\max , l}=\left(I_{\boldsymbol{\lambda}, 1}^{\max , l}, \ldots, I_{\boldsymbol{\lambda}, l}^{\max , l}\right) .
$$

We fix $i, j$ until the end of the proof of Proposition 6.6, and omit the condition $|\boldsymbol{\lambda}|=n$.

Lemma 6.7. Assume that formula (6.19) holds for $\boldsymbol{\lambda}=\left(0, \ldots, 0, \lambda_{k}, \ldots, \lambda_{N}\right)$ with $k \leqslant i$. Then formula (6.19) holds for $\tilde{\boldsymbol{\lambda}}=\left(0, \ldots, 0,1, \lambda_{k}, \ldots, \lambda_{N}\right)$.

Proof. Formula (6.19) for $\boldsymbol{\lambda}$ has the form

$$
\begin{aligned}
\left(\sum_{l \in I_{\boldsymbol{\lambda}, i}^{\max , j}} t_{l}^{(j)}-\right. & \left.\sum_{l \in I_{\boldsymbol{\lambda}, i}^{\max }, j+1} t_{l}^{(j+1)}\right) \operatorname{Sym}_{\boldsymbol{t}^{(k)}} \ldots \operatorname{Sym}_{\boldsymbol{t}^{(N-1)}}\left(U_{I_{\boldsymbol{\lambda}}^{\max }}(\boldsymbol{t})\right) \\
& =\operatorname{Sym}_{\boldsymbol{t}^{(k)}} \ldots \operatorname{Sym}_{\boldsymbol{t}^{(N-1)}}\left(C_{\boldsymbol{\lambda}, i, j+1} \sum_{a=1}^{\lambda_{i}} U_{\left(I_{\boldsymbol{\lambda}}^{\max )_{i, j+1}^{\prime}}\right.}(\boldsymbol{t})\right)
\end{aligned}
$$

where $C_{\boldsymbol{\lambda}, i, j+1}$ is the factor in the second and third lines of definition (4.5).

In addition to the variables $\boldsymbol{t}=\left(\boldsymbol{t}^{(k)}, \ldots, \boldsymbol{t}^{(N)}\right)$ appearing in formula (6.20), formula (6.19) for $\tilde{\boldsymbol{\lambda}}$ contains the new variables $\boldsymbol{t}_{\text {new }}=\left(t_{1}^{(k-1)}, t_{1+\lambda^{(k)}}^{(k)}, t_{1+\lambda^{(k+1)}}^{(k+1)}, \ldots, t_{1+\lambda^{(N-1)}}^{(N-1)}, t_{1+\lambda(N)}^{(N)}\right)$, and has the form

$$
\begin{aligned}
\left(\sum_{l \in I_{\tilde{\boldsymbol{\lambda}}, i}^{\max , j}} t_{l}^{(j)}-\right. & \left.\sum_{l \in I_{\tilde{\boldsymbol{\lambda}}, i}^{\max , j+1}} t_{l}^{(j+1)}\right) \operatorname{Sym}_{\tilde{\boldsymbol{t}}^{(k)}} \ldots \operatorname{Sym}_{\tilde{\boldsymbol{t}}^{(N-1)}}\left(U_{I_{\tilde{\boldsymbol{\lambda}}}^{\max }}(\tilde{\boldsymbol{t}})\right) \\
& =\operatorname{Sym}_{\tilde{\boldsymbol{t}}^{(k)}} \ldots \operatorname{Sym}_{\tilde{\boldsymbol{t}}^{(N-1)}}\left(C_{\tilde{\boldsymbol{\lambda}}, i, j+1} \sum_{a=1}^{\lambda_{i}} U_{\left(I_{\tilde{\boldsymbol{\lambda}}}^{\max )_{i, j+1}^{\prime}}\right.}(\tilde{\boldsymbol{t}})\right)
\end{aligned}
$$


where $\tilde{\boldsymbol{t}}=\boldsymbol{t} \cup \boldsymbol{t}_{\text {new }}=\left(\tilde{\boldsymbol{t}}^{(k-1)}, \tilde{\boldsymbol{t}}^{(k)}, \ldots, \tilde{\boldsymbol{t}}^{(N)}\right)$. It is easy to see from definition (3.6) that

$$
U_{I_{\tilde{\lambda}}^{\max }}(\tilde{\boldsymbol{t}})=U_{I_{\lambda}^{\max }}(\boldsymbol{t}) F(\tilde{\boldsymbol{t}})
$$

where $F(\tilde{\boldsymbol{t}})$ is the product of all factors appearing in (3.6) involving the interrelation of two variables at least one of those being from $\boldsymbol{t}_{\text {new }}$. Moreover, $F(\tilde{\boldsymbol{t}})$ is symmetric in the variables $\boldsymbol{t}^{(l)}$ for each $l=k, \ldots, N$. Furthermore, since $I_{\boldsymbol{\lambda}, i}^{\max , j}=I_{\tilde{\boldsymbol{\lambda}}, i}^{\max , j}$ and $I_{\boldsymbol{\lambda}, i}^{\max , j+1}=I_{\tilde{\boldsymbol{\lambda}}, i}^{\max , j+1}$, the first factors in the left-hand sides of formulas (6.20) and (6.21) coincide.

By all these observations, to get formula (6.21) from (6.20), we need to verify that

$$
\operatorname{Sym}_{\tilde{\boldsymbol{t}}^{(k)}} \ldots \operatorname{Sym}_{\tilde{\boldsymbol{t}}^{(N-1)}}\left(C_{\tilde{\boldsymbol{\lambda}}, i, j+1} \sum_{a=1}^{\lambda_{i}} U_{\left(I_{\tilde{\boldsymbol{\lambda}}}^{\max }\right)_{i, j+1}^{\prime a}}-F C_{\boldsymbol{\lambda}, i, j+1} \sum_{a=1}^{\lambda_{i}} U_{\left(I_{\boldsymbol{\lambda}}^{\max }\right)_{i, j+1}^{\prime a}}\right)=0 .
$$

This equality follows from identity $(3.12)$ for the variables $t_{1+\lambda^{(i-1)}}^{(i-1)}, t_{\lambda^{(i)}}^{(i)}, t_{1+\lambda^{(i)}}^{(i)}, \ldots, t_{\lambda^{(j)}}^{(j)}$, $t_{1+\lambda^{(j)}}^{(j)}, t_{1+\lambda^{(j+1)}}^{(j+1)}$. Lemma 6.7 is proved.

Example. Let $N=5, \boldsymbol{\lambda}=(0,0,1,0,0), \tilde{\boldsymbol{\lambda}}=(1,0,1,0,0)$. For $i=3, j=3$, formulas (6.20) and (6.23) take the form $t_{1}^{(3)}-t_{1}^{(4)}=t_{1}^{(3)}-t_{1}^{(4)}$ and

$$
\begin{aligned}
\operatorname{Sym}_{t_{1}^{(3)}, t_{2}^{(3)}} \operatorname{Sym}_{t_{1}^{(4)}, t_{2}^{(4)}}\left(\left(t_{1}^{(3)}-t_{1}^{(4)}\right)\left(t_{1}^{(2)}-t_{1}^{(3)}\right)\left(t_{1}^{(3)}-t_{2}^{(4)}-h\right)\left(t_{2}^{(3)}-t_{1}^{(4)}\right)\right. \\
\left.\times\left(t_{1}^{(4)}-t_{2}^{(5)}-h\right)\left(t_{2}^{(4)}-t_{1}^{(5)}\right) \frac{t_{2}^{(3)}-t_{1}^{(3)}-h}{t_{2}^{(3)}-t_{1}^{(3)}} \frac{t_{2}^{(4)}-t_{1}^{(4)}-h}{t_{2}^{(4)}-t_{1}^{(4)}}\right) \\
=\operatorname{Sym}_{t_{1}^{(3)}, t_{2}^{(3)}} \operatorname{Sym}_{t_{1}^{(4)}, t_{2}^{(4)}}\left(\left(t_{1}^{(3)}-t_{2}^{(4)}-h\right)\left(t_{2}^{(2)}-t_{1}^{(3)}\right)\left(t_{2}^{(3)}-t_{2}^{(4)}\right)\left(t_{1}^{(3)}-t_{1}^{(4)}\right)\right. \\
\left.\times\left(t_{1}^{(4)}-t_{2}^{(5)}-h\right)\left(t_{2}^{(4)}-t_{1}^{(5)}\right) \frac{t_{2}^{(3)}-t_{1}^{(3)}-h}{t_{2}^{(3)}-t_{1}^{(3)}} \frac{t_{2}^{(4)}-t_{1}^{(4)}-h}{t_{2}^{(4)}-t_{1}^{(4)}}\right),
\end{aligned}
$$

respectively. The last equality follows from identity (3.12) for the variables $t_{1}^{(2)}, t_{1}^{(3)}, t_{2}^{(3)}$, $t_{2}^{(4)}$.

For $i=3, j=4$, formulas (6.20) and (6.23) take the form $t_{1}^{(4)}-t_{1}^{(5)}=t_{1}^{(4)}-t_{1}^{(5)}$ and

$$
\begin{aligned}
\operatorname{Sym}_{t_{1}^{(3)}, t_{2}^{(3)}} \operatorname{Sym}_{t_{1}^{(4)}, t_{2}^{(4)}}\left(\left(t_{1}^{(4)}-t_{1}^{(5)}\right)\left(t_{1}^{(2)}-t_{1}^{(3)}\right)\left(t_{1}^{(3)}-t_{2}^{(4)}-h\right)\left(t_{2}^{(3)}-t_{1}^{(4)}\right)\right. \\
\left.\times\left(t_{1}^{(4)}-t_{2}^{(5)}-h\right)\left(t_{2}^{(4)}-t_{1}^{(5)}\right) \frac{t_{2}^{(3)}-t_{1}^{(3)}-h}{t_{2}^{(3)}-t_{1}^{(3)}} \frac{t_{2}^{(4)}-t_{1}^{(4)}-h}{t_{2}^{(4)}-t_{1}^{(4)}}\right) \\
=\operatorname{Sym}_{t_{1}^{(3)}, t_{2}^{(3)}} \operatorname{Sym}_{t_{1}^{(4)}, t_{2}^{(4)}}\left(\left(t_{1}^{(2)}-t_{2}^{(3)}-h\right)\left(t_{2}^{(2)}-t_{1}^{(3)}\right)\left(t_{1}^{(3)}-t_{2}^{(4)}-h\right)\left(t_{2}^{(4)}-t_{1}^{(5)}\right)\right. \\
\left.\times\left(t_{2}^{(4)}-t_{2}^{(5)}\right)\left(t_{1}^{(4)}-t_{1}^{(5)}\right) \frac{t_{2}^{(3)}-t_{1}^{(3)}-h}{t_{2}^{(3)}-t_{1}^{(3)}} \frac{t_{2}^{(4)}-t_{1}^{(4)}-h}{t_{2}^{(4)}-t_{1}^{(4)}}\right),
\end{aligned}
$$


respectively. The last equality follows from identity (3.12) for the variables $t_{1}^{(2)}, t_{1}^{(3)}, t_{2}^{(3)}$, $t_{1}^{(4)}, t_{2}^{(4)}, t_{2}^{(5)}$.

Lemma 6.8. Assume that formula (6.19) holds for $\boldsymbol{\lambda}=\left(0, \ldots, 0, \lambda_{k}, \ldots, \lambda_{N}\right)$ with $k<i$ and $\lambda_{k}>0$. Then formula (6.19) holds for $\tilde{\boldsymbol{\lambda}}=\left(0, \ldots, 0,0, \lambda_{k}+1, \ldots, \lambda_{N}\right)$.

Proof. The proof is completely similar to that of Lemma 6.7. The only change is that the new variables are $\boldsymbol{t}_{\text {new }}=\left(t_{1+\lambda^{(k)}}^{(k)}, t_{1+\lambda^{(k+1)}}^{(k+1)}, \ldots, t_{1+\lambda^{(N-1)}}^{(N-1)}, t_{1+\lambda^{(N)}}^{(N)}\right)$.

Example. Let $N=3, \boldsymbol{\lambda}=(1,1,0), \tilde{\boldsymbol{\lambda}}=(2,1,0)$. For $i=2, j=2$, formula (6.23) proof follows from identity (3.12) for the variables $t_{2}^{(1)}, t_{2}^{(2)}, t_{3}^{(2)}, t_{3}^{(3)}$.

Lemmas 6.7 and 6.8 yield Proposition 6.6.

Theorem 4.4 for $i>1$, arbitrary $N, \boldsymbol{\lambda}$, and $I=I^{\max }$ is proved.

6.7. Modification of the three-term relation. For integers $\alpha, \beta, 1 \leqslant \alpha<\beta \leqslant N$, and $\boldsymbol{\lambda} \in \mathbb{Z}_{\geqslant 0}^{N},|\boldsymbol{\lambda}|=n$, recall the notations $\boldsymbol{t}^{\{\alpha, \beta\}}, \boldsymbol{t}_{\{\alpha, \beta\}}, \boldsymbol{\lambda}_{\alpha, \beta}$, in Sections 4.3 and 4.4 .

Lemma 6.9. For any $1 \leqslant \alpha<\beta \leqslant N$ and $I \in \mathcal{I}_{\boldsymbol{\lambda}_{\alpha, \beta}}$, we have $\check{d}_{\{\alpha, \beta\}} U_{I}=c_{\alpha, \beta} \check{d}_{\{\alpha, \beta\}} W_{I}$, where $c_{\alpha, \beta}=\frac{\prod_{i=\alpha}^{\beta-1} \lambda^{(i)}}{\prod_{i=1}^{N-1} \lambda^{(i) !}}$.

Proof. Let

$$
\begin{aligned}
G_{\alpha, \beta}(\boldsymbol{t}, \boldsymbol{z}) & =\left(t_{\lambda^{(\alpha-1)}}^{(\alpha-1)}-t_{\lambda^{(\alpha)}}^{(\alpha)}-h\right) \prod_{i=\alpha}^{\beta-1} \prod_{a=1}^{\lambda^{(i-1)}-1}\left(t_{a}^{(i-1)}-t_{\lambda^{(i)}}^{(i)}-h\right) \times \\
& \times\left(t_{\lambda^{(\beta-1)}}^{(\beta-1)}-t_{\lambda^{(\beta)}}^{(\beta)}\right) \prod_{i=\alpha}^{\beta-1} \prod_{a=1}^{\lambda^{(i+1)}-1}\left(t_{\lambda^{(i)}}^{(i)}-t_{a}^{(i+1)}\right) \prod_{i=\alpha}^{\beta-1} \prod_{a=1}^{\lambda^{(i)}-1} \frac{t_{\lambda^{(i)}}^{(i)}-t_{a}^{(i)}-h}{t_{\lambda^{(i)}}^{(i)}-t_{a}^{(i)}}
\end{aligned}
$$

be the product in the right-hand side of formula (4.5). Since $G_{\alpha, \beta}(\boldsymbol{t}, \boldsymbol{z})$ is symmetric in the variables $\boldsymbol{t}_{\{\alpha, \beta\}}^{(i)}$ for every $i=1, \ldots, N-1$, we can apply the symmetrization in those variables to $U_{I}\left(\boldsymbol{t}_{\{\alpha, \beta\}}, \boldsymbol{z}\right)$ and divide the result by the order of the relevant product of the symmetric groups before doing the overall symmetrization in formula (4.8) for $\check{d}_{\{\alpha, \beta\}} U_{I}$. This results in replacing $U_{I}\left(\boldsymbol{t}_{\{\alpha, \beta\}}, \boldsymbol{z}\right)$ by $c_{\alpha, \beta} W_{I}\left(\boldsymbol{t}_{\{\alpha, \beta\}}, \boldsymbol{z}\right)$, see formula (3.5).

Recall the operator $S_{i, i+1}$ acting on functions of $z_{1}, \ldots, z_{n}$ given by formula (3.7).

Lemma 6.10. For any $i=1, \ldots, n-1,1 \leqslant \alpha<\beta \leqslant N$, and $I \in \mathcal{I}_{\boldsymbol{\lambda}_{\alpha, \beta}}$, we have

$$
S_{i, i+1}\left(\check{d}_{\{\alpha, \beta\}} U_{I}\right)=\check{d}_{\{\alpha, \beta\}} U_{s_{i, i+1}(I)} .
$$

Proof. The product $G_{\alpha, \beta}(\boldsymbol{t}, \boldsymbol{z})$, see $(6.24)$, is symmetric in $z_{1}, \ldots, z_{n}$. Hence

$$
\begin{array}{r}
S_{i, i+1}\left(\check{d}_{\{\alpha, \beta\}} U_{I}\right)=c_{\alpha, \beta} S_{i, i+1}\left(\check{d}_{\{\alpha, \beta\}} W_{I}\right)=c_{\alpha, \beta} \check{d}_{\{\alpha, \beta\}}\left(S_{i, i+1}\left(W_{I}\right)\right) \\
=c_{\alpha, \beta} \check{d}_{\{\alpha, \beta\}} W_{s_{i, i+1}(I)}=\check{d}_{\{\alpha, \beta\}} U_{s_{i, i+1}(I)} .
\end{array}
$$

by Lemmas 6.9 and 3.2 . 
6.8. The end of the proof of Theorem 4.4. Given $l, 1 \leqslant l \leqslant N-1$, we add formulas (4.14) for $i=1, \ldots, l$. The result is

$$
\begin{aligned}
& \left(\sum_{j=1}^{\lambda^{(l)}} t_{j}^{(l)}-\sum_{i=1}^{l} \sum_{a \in I_{i}} z_{a}\right) W_{I}+h \sum_{i=1}^{l} \sum_{j=l+1}^{N} \sum_{m_{1}=1}^{\lambda_{i}} \sum_{\substack{m_{2}=1 \\
\ell_{i, m_{1}}<\ell_{j, m_{2}}}}^{\lambda_{j}} W_{I_{i, j}, m_{1}, m_{2}} \\
& =\sum_{i=1}^{l} \sum_{j=l+1}^{N} \sum_{a=1}^{\lambda_{i}} \check{d}_{\{i, j\}} U_{(I)_{i, j}^{a}},
\end{aligned}
$$

To finish the proof of Theorem 4.4, we need to prove formula (6.26) for any $I$ and any $i=1$, ..., $N-1$.

For any permutation $\sigma$, denote by $|\sigma|$ the length of $\sigma$. For any $J, J^{\prime} \in \mathcal{I}_{\boldsymbol{\lambda}}$, define the permutation $\sigma_{J, J^{\prime}}$ as follows: if $J_{m}=\left\{j_{m, 1}<\ldots<j_{m, \lambda_{m}}\right\}, J_{m}^{\prime}=\left\{j_{m, 1}^{\prime}<\ldots<j_{m, \lambda_{m}}^{\prime}\right\}$, then $\sigma_{J, J^{\prime}}\left(j_{m, l}^{\prime}\right)=j_{m, l}$. Set $\sigma_{J}=\sigma_{J, I_{\max }}$. The permutation $\sigma_{J}$ has the minimal length amongst all permutations $\sigma$ such that $\sigma\left(I^{\max }\right)=J$.

Lemma 6.11. Assume that for $J \in \mathcal{I}_{\boldsymbol{\lambda}}$ and a transposition $s_{i, i+1}$, we have $\left|s_{i, i+1} \sigma_{J}\right|<\left|\sigma_{J}\right|$. Then $s_{i, i+1} \sigma_{J}=\sigma_{s_{i, i+1}(J)}$.

We will prove formula $(6.26)$ by induction with respect to the length of $\sigma_{I}$. For the base of induction $I=I^{\max }$, formula (6.26) is proved already.

Fix $I \in \mathcal{I}_{\boldsymbol{\lambda}}$ and find $m$ such that $\left|s_{m, m+1} \sigma_{I}\right|<\left|\sigma_{I}\right|$. Let $p, r$ be such that $m \in I_{p}$ and $m+1 \in I_{r}$. Since $\left|s_{m, m+1} \sigma_{I}\right|<\left|\sigma_{I}\right|$, we have $p<r$.

Denote $\tilde{I}=s_{m, m+1}(I)$. Then $\tilde{I}_{p}=I_{p}-\{m\} \cup\{m+1\}, \tilde{I}_{r}=I_{r}-\{m+1\} \cup\{m\}$, and $\tilde{I}_{c}=I_{c}$, otherwise. And clearly, $I=s_{m, m+1}(\tilde{I})$.

Write formula (6.26) for $\tilde{I}$ :

$$
\begin{array}{r}
\left(\sum_{j=1}^{\lambda^{(l)}} t_{j}^{(l)}-\sum_{i=1}^{l} \sum_{a \in \tilde{I}_{i}} z_{a}\right) W_{\tilde{I}}+h \sum_{i=1}^{l} \sum_{j=l+1}^{N} \sum_{m_{1}=1}^{\lambda_{i}} \sum_{\tilde{\ell}_{m_{2}=1}=\tilde{\ell}_{j, m_{2}}}^{\lambda_{j}} W_{\tilde{I}_{i, j ; m_{1}, m_{2}}} \\
=\sum_{i=1}^{l} \sum_{j=l+1}^{N} \sum_{a=1}^{\lambda_{i}} \check{d}_{\{i, j\}} U_{(\tilde{I})_{i, j}^{a}},
\end{array}
$$

where $\tilde{I}_{c}=\left(\tilde{\ell}_{c, 1}, \ldots, \tilde{\ell}_{c, \lambda_{c}}\right)$. We will show that applying the operator $S_{m, m+1}$ to both sides of formula (6.27) transforms it to formula (6.26) for $I$.

To compare the right-hand sides, observe that $s_{m, m+1}\left((\tilde{I})_{i, j}^{a}\right)=(I)_{i, j}^{a}$. Hence, Lemma 6.10 yields $S_{m, m+1}\left(\breve{d}_{\{i, j\}} U_{(\tilde{I})_{i, j}^{a}}\right)=\breve{d}_{\{i, j\}} U_{(I)_{i, j}^{a}}$, that proves the desired assertion.

To compare the left-hand sides, observe first that

and

$$
s_{m, m+1}\left(\tilde{I}_{i, j ; m_{1}, m_{2}}\right)=I_{i, j ; s_{m, m+1}\left(m_{1}\right), s_{m, m+1}\left(m_{2}\right)}
$$

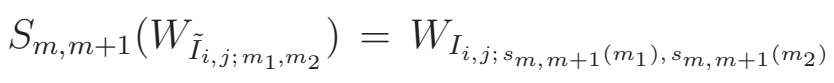


by Lemma 3.2. This proves the desired transformation of the second sum in the left-hand side of (6.27) term by term provided $p>l$ or $r \leqslant l$. If $p \leqslant l<r$, the matching between the terms of the second sums in (6.27) and (6.26) is not perfect and the sum in (6.26) contains one more term $h W_{I_{p, r ; m, m+1}}$.

If $p>l$ or $r \leqslant l$, the sum $\sum_{i=1}^{l} \sum_{a \in \tilde{I}_{i}} z_{a}$ in formula (6.27) is symmetric in $z_{m}, z_{m+1}$ and equals the sum $\sum_{i=1}^{l} \sum_{a \in I_{i}} z_{a}$ in formula (6.26). Thus

$$
\begin{aligned}
S_{m, m+1}\left(\left(\sum_{j=1}^{\lambda^{(l)}} t_{j}^{(l)}-\sum_{i=1}^{l} \sum_{a \in \tilde{I}_{i}} z_{a}\right) W_{\tilde{I}}\right) & =\left(\sum_{j=1}^{\lambda^{(l)}} t_{j}^{(l)}-\sum_{i=1}^{l} \sum_{a \in I_{i}} z_{a}\right) S_{m, m+1}\left(W_{\tilde{I}}\right) \\
& =\left(\sum_{j=1}^{\lambda^{(l)}} t_{j}^{(l)}-\sum_{i=1}^{l} \sum_{a \in I_{i}} z_{a}\right) W_{I}
\end{aligned}
$$

by Lemma 3.2. If $p \leqslant l<r$, then we have

$$
\begin{aligned}
S_{m, m+1}\left(\left(\sum_{j=1}^{\lambda^{(l)}} t_{j}^{(l)}-\sum_{i=1}^{l} \sum_{a \in \tilde{I}_{i}} z_{a}\right) W_{\tilde{I}}\right) & =\left(\sum_{j=1}^{\lambda^{(l)}} t_{j}^{(l)}-\sum_{i=1}^{l} \sum_{a \in I_{i}} z_{a}\right) S_{m, m+1}\left(W_{\tilde{I}}\right)+h W_{\tilde{I}} \\
& =\left(\sum_{j=1}^{\lambda^{(l)}} t_{j}^{(l)}-\sum_{i=1}^{l} \sum_{a \in I_{i}} z_{a}\right) W_{I}+h W_{I_{p, r ; m, m+1}}
\end{aligned}
$$

since $\tilde{I}=I_{p, r ; m, m+1}$. This shows that the operator $S_{m, m+1}$ transforms formula (6.27) to formula (6.26). This completes the induction step. Theorem 4.4 is proved.

Example. Let $N=2, n=3, \boldsymbol{\lambda}=(2,1), I=(\{1,3\},\{2\}), I^{\max }=(\{2,3\},\{1\}), \sigma_{I}=$ $s_{1,2}$. Formula $(6.26)$ is

$$
\left(t_{1}^{(1)}+t_{2}^{(1)}-z_{1}-z_{3}\right) W_{\{1,3\},\{2\}}+h W_{\{2,3\}\{1\}}=\check{d}_{\{1,2\}}\left(U_{\{1\},\{2,3\}}+U_{\{3\},\{1,2\}}\right),
$$

formula $(6.27)$ is

$$
\left(t_{1}^{(1)}+t_{2}^{(1)}-z_{2}-z_{3}\right) W_{\{2,3\},\{2\}}=\check{d}_{\{1,2\}}\left(U_{\{2\},\{1,3\}}+U_{\{3\},\{1,2\}}\right) .
$$

and the operator $S_{1,2}$ transforms formula (6.29) to formula (6.28).

\section{Corollary of Theorems 4.3 And 4.4}

Let $\boldsymbol{\lambda} \in \mathbb{Z}_{\geqslant 0}^{N},|\boldsymbol{\lambda}|=n$, and $I \in \mathcal{I}_{\boldsymbol{\lambda}}$. Recall the notations $(I)_{\alpha, \beta}^{a}, I_{i, j ; m_{1}, m_{2}}$, see $(4.11)$, (4.13), and the discrete differentials $d_{\{\alpha, \beta\}} g$, see (4.6). Define the discrete differential

$$
D_{I, i}=\sum_{j=i+1}^{N} \sum_{a=1}^{\lambda_{j}} d_{\{j, i\}} U_{(I)_{j, i}^{a}}-\sum_{j=1}^{i-1} \sum_{a=1}^{\lambda_{i}} d_{\{i, j\}} U_{(I)_{i, j}^{a}}
$$


Corollary 7.1. We have

$$
\begin{aligned}
& \left(\sum_{j=1}^{\lambda^{(i)}} t_{j}^{(i)}-\sum_{j=1}^{\lambda^{(i-1)}} t_{j}^{(i-1)}-\sum_{a \in I_{i}} z_{a}\right) W_{I}+h\left(\sum_{j=1}^{i-1} \frac{q_{i} \lambda_{j}}{q_{i}-q_{j}}+\sum_{j=i+1}^{N} \frac{q_{j} \lambda_{i}}{q_{i}-q_{j}}\right) W_{I}+ \\
& +h \sum_{\substack{j=1 \\
j \neq i}}^{N} \sum_{m_{1}=1}^{\lambda_{i}}\left(\sum_{\substack{m_{2}=1 \\
\ell_{i, m_{1}}<\ell_{j, m_{2}}}}^{\lambda_{j}} W_{I_{i, j ; m_{1}, m_{2}}}+\frac{q_{j}}{q_{i}-q_{j}} \sum_{m_{2}=1}^{\lambda_{j}} W_{I_{i, j ; m_{1}, m_{2}}}\right)=D_{I, i} .
\end{aligned}
$$

Proof. Theorems 4.3 and 4.4, and formula (4.9) imply that

$$
\begin{aligned}
& \left(\sum_{j=1}^{\lambda^{(i)}} t_{j}^{(i)}-\sum_{j=1}^{\lambda^{(i-1)}} t_{j}^{(i-1)}-\sum_{a \in I_{i}} z_{a}\right) W_{I}-h \sum_{j=1}^{i-1} \sum_{m_{1}=1}^{\lambda_{i}} \sum_{\substack{m_{2}=1 \\
\ell_{i, m_{1}}>\ell_{j, m_{2}}}}^{\lambda_{j}} W_{I_{i, j ; m_{1}, m_{2}}} \\
& +h \sum_{j=i+1}^{N} \sum_{m_{1}=1}^{\lambda_{i}} \sum_{\substack{m_{2}=1 \\
\ell_{i, m_{1}}<\ell_{j, m_{2}}}}^{\lambda_{j}} W_{I_{i, j ; m_{1}, m_{2}}}+h \sum_{j=1}^{i-1} \frac{q_{i}}{q_{i}-q_{j}}\left(\lambda_{j} W_{I}+\sum_{m_{1}=1}^{\lambda_{i}} \sum_{m_{2}=1}^{\lambda_{j}} W_{I_{i, j ; m_{1}, m_{2}}}\right) \\
& +h \sum_{j=i+1}^{N} \frac{q_{j}}{q_{i}-q_{j}}\left(\lambda_{i} W_{I}+\sum_{m_{1}=1}^{\lambda_{i}} \sum_{m_{2}=1}^{\lambda_{j}} W_{I_{i, j ; m_{1}, m_{2}}}\right)=D_{I, i} .
\end{aligned}
$$

Formula (7.2) is obtained now by rearranging the terms in the left-hand side of this equality.

Recall the scalar master function $\Phi_{\boldsymbol{\lambda}}(\boldsymbol{t} ; \boldsymbol{z} ; h ; \boldsymbol{q})$ given by (4.1). Define

$$
\Omega_{\boldsymbol{\lambda}}(\boldsymbol{q})=\prod_{i=1}^{N-1} \prod_{j=i+1}^{N}\left(1-q_{j} / q_{i}\right)^{h \lambda_{i} / \kappa} .
$$

Introduce the $\left(\mathbb{C}^{N}\right)_{\lambda}^{\otimes n}$-valued weight function

$$
W_{\boldsymbol{\lambda}}(\boldsymbol{t} ; \boldsymbol{z} ; h)=\sum_{I \in \mathcal{I}_{\boldsymbol{\lambda}}} W_{I}(\boldsymbol{t} ; \boldsymbol{z} ; h) v_{I} .
$$

Recall the dynamical Hamiltonians $X_{i}(\boldsymbol{z} ; h ; \boldsymbol{q})$ defined in $(2.1)$.

Theorem 7.2. For every $i=1, \ldots, N$, we have

$$
\begin{aligned}
\left(\kappa q_{i} \frac{\partial}{\partial q_{i}}-X_{i}(\boldsymbol{z} ; h ; \boldsymbol{q})\right) & \Omega_{\boldsymbol{\lambda}}(\boldsymbol{q}) \Phi_{\boldsymbol{\lambda}}(\boldsymbol{t} ; \boldsymbol{z} ; h ; \boldsymbol{q}) W_{\boldsymbol{\lambda}}(\boldsymbol{t} ; \boldsymbol{z})= \\
= & \Omega_{\boldsymbol{\lambda}}(\boldsymbol{q}) \Phi_{\boldsymbol{\lambda}}(\boldsymbol{t} ; \boldsymbol{z} ; h ; \boldsymbol{q}) \sum_{I \in \mathcal{I}_{\boldsymbol{\lambda}}} D_{I, i}(\boldsymbol{t} ; \boldsymbol{z} ; h ; \boldsymbol{q}) v_{I}
\end{aligned}
$$

Proof. The statement is equivalent to Corollary 7.1. 


\section{INTEGRAL REPRESENTATIONS FOR SOLUTIONS OF DYNAMICAL EQUATIONS}

8.1. Formal integrals. Let $\boldsymbol{\lambda} \in \mathbb{Z}_{\geqslant 0}^{N},|\boldsymbol{\lambda}|=n$, and $\kappa \in \mathbb{C}^{\times}$. Consider the space of functions of the form $\Phi_{\boldsymbol{\lambda}}(\boldsymbol{t} ; \boldsymbol{z} ; h ; \boldsymbol{q}) f(\boldsymbol{t} ; \boldsymbol{z} ; h ; \boldsymbol{q})$, where $\Phi_{\boldsymbol{\lambda}}(\boldsymbol{t} ; \boldsymbol{z} ; h ; \boldsymbol{q})$ is the master function (4.1), and $f(\boldsymbol{t} ; \boldsymbol{z} ; h ; \boldsymbol{q})$ is a polynomial in $\boldsymbol{t}$ and holomorphic function of $\boldsymbol{z}, h, \boldsymbol{q}$ on some domain $L \subset \mathbb{C}^{n} \times \mathbb{C} \times \mathbb{C}^{N}$. Assume that we have a map $\mathcal{M}$ assigning to a function $\Phi_{\boldsymbol{\lambda}} f$ a function $\mathcal{M}\left(\Phi_{\boldsymbol{\lambda}} f\right)$ of variables $\boldsymbol{z}, h, \boldsymbol{q}$, holomorphic on $L$, such that:

(i) The map $\mathcal{M}$ is linear over the field of meromorphic on $L$ functions in $\boldsymbol{z}, \boldsymbol{q}, \mathrm{h}$,

$$
\mathcal{M}\left(\Phi_{\boldsymbol{\lambda}}\left(g_{1} f_{1}+g_{2} f_{2}\right)\right)=g_{1} \mathcal{M}\left(\Phi_{\boldsymbol{\lambda}} f_{1}\right)+g_{2} \mathcal{M}\left(\Phi_{\boldsymbol{\lambda}} f_{2}\right)
$$

for any meromorphic functions $g_{1}, g_{2}$ of $\boldsymbol{z}, h, \boldsymbol{q}$, such that $g_{1} f_{1}$ and $g_{2} f_{2}$ are holomorphic on $L$.

(ii) For any $i=1, \ldots, N$, we have

$$
\frac{\partial}{\partial q_{i}} \mathcal{M}\left(\Phi_{\boldsymbol{\lambda}} f\right)=\mathcal{M}\left(\frac{\partial}{\partial q_{i}}\left(\Phi_{\boldsymbol{\lambda}} f\right)\right)
$$

(iii) If $f$ is a discrete differential of a polynomial in $t$, then

$$
\mathcal{M}\left(\Phi_{\lambda} f\right)=0 .
$$

A map $\mathcal{M}$ is called a formal integral. We have the following corollary of Theorem 7.2.

Lemma 8.1. If $\mathcal{M}$ is a formal integral, then the $\left(\mathbb{C}^{N}\right)_{\lambda}^{\otimes n}$-valued function

$$
F_{\mathcal{M}}(\boldsymbol{z} ; h ; \boldsymbol{q}):=\Omega_{\boldsymbol{\lambda}} \mathcal{M}\left(\Phi_{\boldsymbol{\lambda}} W_{\boldsymbol{\lambda}}\right)=\Omega_{\boldsymbol{\lambda}} \sum_{I \in \mathcal{I}_{\boldsymbol{\lambda}}} \mathcal{M}\left(\Phi_{\boldsymbol{\lambda}} W_{I}\right) v_{I}
$$

holomorphic on $L$, is a solution of the dynamical differential equations (2.3).

8.2. Jackson integral. Consider the space $\mathbb{C}^{\lambda\{1\}} \times \mathbb{C}^{n} \times \mathbb{C} \times \mathbb{C}^{N}$ with coordinates $\boldsymbol{t}, \boldsymbol{z}, h, \boldsymbol{q}$. The lattice $\kappa \mathbb{Z}^{\lambda^{\{1\}}}$ naturally acts on this space by shifting the $\boldsymbol{t}$-coordinates.

Let $J=\left(J_{1}, \ldots, J_{N}\right) \in \mathcal{I}_{\lambda}$. Recall the notation $\bigcup_{i=1}^{k} J_{i}=\left\{j_{1}^{(k)}<\ldots<j_{\lambda^{(k)}}^{(k)}\right\}$. Define $\Sigma_{J} \subset \mathbb{C}^{\lambda\{1\}} \times \mathbb{C}^{n} \times \mathbb{C} \times \mathbb{C}^{N}$ by the equations:

$$
t_{i}^{(k)}=z_{j_{i}^{(k)}}, \quad k=1, \ldots, N-1, \quad i=1, \ldots, \lambda^{(k)},
$$

and call it a discrete cycle.

For a function of $\boldsymbol{t}$ and a point $s \in \mathbb{C}^{\lambda^{\{1\}}}$, define $\operatorname{Res}_{\boldsymbol{t}=\boldsymbol{s}}$ to be the iterated residue,

$$
\operatorname{Res}_{t=s}=\operatorname{Res}_{t_{1}^{(1)}=s_{1}^{(1)}} \ldots \operatorname{Res}_{t_{\lambda(1)}^{(1)}=s_{\lambda^{(1)}}^{(1)}} \ldots \operatorname{Res}_{t_{1}^{(N-1)}=s_{1}^{(N-1)}} \ldots \operatorname{Res}_{t_{\lambda^{(N-1)}}^{(N-1)}=s_{\lambda^{(N-1)}}^{(N-1)}} .
$$

Let $L^{\prime}$ be the complement in $\mathbb{C}^{n} \times \mathbb{C}$ of the union of the hyperplanes

$$
h=m \kappa, \quad z_{a}-z_{b}=m \kappa, \quad z_{a}-z_{b}+h=m \kappa,
$$

for all $a, b=1, \ldots, n, a \neq b$, and all $m \in \mathbb{Z}$. Let $L^{\prime \prime} \subset \mathbb{C}^{N}$ be the domain

$$
\left|q_{i+1} / q_{i}\right|<1, \quad i=1, \ldots, N-1,
$$


with additional cuts fixing a branch of $\log q_{i}$ for all $i=1, \ldots, N$. Set $L=L^{\prime} \times L^{\prime \prime} \subset$ $\mathbb{C}^{n} \times \mathbb{C} \times \mathbb{C}^{N}$.

Let $f(\boldsymbol{t} ; \boldsymbol{z} ; h ; \boldsymbol{q})$ be a polynomial in $\boldsymbol{t}$ and a holomorphic function of $\boldsymbol{z} ; h ; \boldsymbol{q}$ on $L$. For $(\boldsymbol{z} ; h ; \boldsymbol{q}) \in L$, define

$$
\mathcal{M}_{J}\left(\Phi_{\boldsymbol{\lambda}} f\right)(\boldsymbol{z} ; h ; \boldsymbol{q})=\sum_{\boldsymbol{r} \in \mathbb{Z}^{\lambda}\{1\}} \operatorname{Res}_{\boldsymbol{t}=\Sigma_{J}+\boldsymbol{r} \kappa}\left(\Phi_{\boldsymbol{\lambda}}(\boldsymbol{t} ; \boldsymbol{z} ; h ; \boldsymbol{q}) f(\boldsymbol{t} ; \boldsymbol{z} ; h ; \boldsymbol{q})\right) .
$$

This sum is called the Jackson integral over the discrete cycle $\Sigma_{J}$.

Lemma 8.2. The map $\mathcal{M}_{J}$ is a formal integral.

Proof. Each term of the sum in formula (8.7) is a holomorphic function on $L^{\prime}$. Moreover, $\operatorname{Res}_{\boldsymbol{t}=\Sigma_{J}+\boldsymbol{r} \kappa}\left(\Phi_{\boldsymbol{\lambda}}(\boldsymbol{t} ; \boldsymbol{z} ; h ; \boldsymbol{q}) f(\boldsymbol{t} ; \boldsymbol{z} ; h ; \boldsymbol{q})=0\right.$ if $\boldsymbol{r} \notin \mathbb{Z}_{\leqslant 0}^{\lambda^{\{1\}}}$. Hence, the sum over $\mathbb{Z}^{\lambda^{\{1\}}}$ reduces to the sum over $\mathbb{Z}_{\leqslant 0}^{\lambda^{\{1\}}}$. The result is similar to a multidimensional hypergeometric series multiplied by some fractional powers of $q_{1}, \ldots, q_{N}$. The obtained sum converges if $\left|q_{i+1} / q_{i}\right|<1$ for all $i=1, \ldots, N-1$, and gives a holomorphic function on $L$.

Properties (8.1) - (8.3) for the map $\mathcal{M}_{J}$ are clear. Lemma 8.2 is proved.

Lemma 8.3. The function $\mathcal{M}_{J}\left(\Phi_{\boldsymbol{\lambda}} f\right)$ analytically continues to the hyperplanes $h=m \kappa$ for $m \in \mathbb{Z}_{>0}$.

Proof. By the proof of Lemma 8.2,

$$
\mathcal{M}_{J}\left(\Phi_{\boldsymbol{\lambda}} f\right)(\boldsymbol{z} ; h ; \boldsymbol{q})=\sum_{\boldsymbol{r} \in \mathbb{Z}_{\leqslant 0}^{\lambda\{\}}} \operatorname{Res}_{\boldsymbol{t}=\Sigma_{J}+\boldsymbol{r} \kappa}\left(\Phi_{\boldsymbol{\lambda}}(\boldsymbol{t} ; \boldsymbol{z} ; h ; \boldsymbol{q}) f(\boldsymbol{t} ; \boldsymbol{z} ; h ; \boldsymbol{q})\right) .
$$

for $(\boldsymbol{z} ; h ; \boldsymbol{q}) \in L$. By inspection, if $h \rightarrow m \kappa, m \in \mathbb{Z}_{>0}$, and $\boldsymbol{r} \in \mathbb{Z}_{\leqslant 0}^{\lambda^{\{1\}}}$, then

$$
\operatorname{Res}_{\boldsymbol{t}=\Sigma_{J}+\boldsymbol{r} \kappa}\left(\Phi_{\boldsymbol{\lambda}}(\boldsymbol{t} ; \boldsymbol{z} ; h ; \boldsymbol{q}) f(\boldsymbol{t} ; \boldsymbol{z} ; h ; \boldsymbol{q})\right) \rightarrow \operatorname{Res}_{\boldsymbol{t}=\Sigma_{J}+\boldsymbol{r} \kappa}\left(\Phi_{\boldsymbol{\lambda}}(\boldsymbol{t} ; \boldsymbol{z} ; m \kappa ; \boldsymbol{q}) f(\boldsymbol{t} ; \boldsymbol{z} ; m \kappa ; \boldsymbol{q})\right) .
$$

Hence

$$
\mathcal{M}_{J}\left(\Phi_{\boldsymbol{\lambda}} f\right)(\boldsymbol{z} ; h ; \boldsymbol{q}) \rightarrow \sum_{\boldsymbol{r} \in \mathbb{Z}_{\leqslant 0}^{\lambda}\{1\}} \operatorname{Res}_{\boldsymbol{t}=\Sigma_{J}+\boldsymbol{r} \kappa}\left(\Phi_{\boldsymbol{\lambda}}(\boldsymbol{t} ; \boldsymbol{z} ; m \kappa ; \boldsymbol{q}) f(\boldsymbol{t} ; \boldsymbol{z} ; m \kappa ; \boldsymbol{q})\right)
$$

since the sum in the right-hand side converges if $\left|q_{i+1} / q_{i}\right|<1$ for all $i=1, \ldots, N-1$.

Remark. For $m \in \mathbb{Z}_{>0}$, the sum $\sum_{\boldsymbol{r} \in \mathbb{Z}^{\lambda}\{1\}} \operatorname{Res}_{\boldsymbol{t}=\Sigma_{J}+\boldsymbol{r} \kappa}\left(\Phi_{\boldsymbol{\lambda}}(\boldsymbol{t} ; \boldsymbol{z} ; m \kappa ; \boldsymbol{q}) f(\boldsymbol{t} ; \boldsymbol{z} ; m \kappa ; \boldsymbol{q})\right)$ diverges, and the function $\mathcal{M}_{J}\left(\Phi_{\boldsymbol{\lambda}} f\right)(\boldsymbol{z} ; m \kappa ; \boldsymbol{q})$ is not given by formula (8.7).

Example. Let $N=2, \boldsymbol{\lambda}=(1, n-1), J=(\{1\},\{2,3, \ldots, n\})$. Then

$$
\Phi_{\boldsymbol{\lambda}}(\boldsymbol{t} ; \boldsymbol{z} ; h ; \boldsymbol{q})=\left(e^{\pi \sqrt{-1}} q_{2}\right)^{\sum_{a=1}^{n} z_{a} / \kappa}\left(e^{\pi \sqrt{-1}(n-2)} \frac{q_{1}}{q_{2}}\right)^{t_{1}^{(1)} / \kappa} \prod_{a=1}^{n} \Gamma\left(\frac{t_{1}^{(1)}-z_{a}}{\kappa}\right) \Gamma\left(\frac{z_{a}-t_{1}^{(1)}+h}{\kappa}\right),
$$

and

$$
\mathcal{M}_{(\{1\},\{2,3, \ldots, n\})}\left(\Phi_{\boldsymbol{\lambda}} f\right)=\sum_{r \in \mathbb{Z}} \operatorname{Res}_{t_{1}^{(1)}=z_{1}+r \kappa}\left(\Phi_{\boldsymbol{\lambda}} f\right)
$$


Nonzero contributions to the sum in the right-hand side of (8.10) come from the poles of $\Gamma\left(\left(t_{1}^{(1)}-z_{1}\right) / \kappa\right)$. Explicitly, the answer is

$$
\begin{aligned}
\mathcal{M}_{(\{1\},\{2,3, \ldots, n\})}\left(\Phi_{\boldsymbol{\lambda}} f\right)=\left(e^{\pi \sqrt{-1}(n-1)} q_{1}\right)^{z_{1} / \kappa}\left(e^{\pi \sqrt{-1}} q_{2}\right)^{\sum_{a=2}^{n} z_{a} / \kappa} \times \\
\times \kappa \Gamma\left(\frac{h}{\kappa}\right) \prod_{a=2}^{n} \Gamma\left(\frac{z_{1}-z_{a}}{\kappa}\right) \Gamma\left(\frac{z_{a}-z_{1}+h}{\kappa}\right) \times \\
\times \sum_{l=0}^{\infty} f\left(z_{1}-l \kappa ; \boldsymbol{z} ; h ; \boldsymbol{q}\right)\left(\frac{q_{2}}{q_{1}}\right)^{l} \prod_{j=0}^{l-1}\left(\frac{h+j \kappa}{\kappa+j \kappa} \prod_{a=2}^{n} \frac{z_{a}-z_{1}+h+j \kappa}{z_{a}-z_{1}+\kappa+j \kappa}\right),
\end{aligned}
$$

and the series converges if $\left|q_{2} / q_{1}\right|<1$.

8.3. Solutions of dynamical equations. Recall the $\left(\mathbb{C}^{N}\right)_{\lambda}^{\otimes n}$-valued weight function $W_{\boldsymbol{\lambda}}(\boldsymbol{t} ; \boldsymbol{z})$, given by (7.4). For $J \in \mathcal{I}_{\boldsymbol{\lambda}}$, define

$$
\Psi_{J}(\boldsymbol{z} ; h ; \boldsymbol{q})=\Omega_{\boldsymbol{\lambda}}(\boldsymbol{q}) \mathcal{M}_{J}\left(\Phi_{\boldsymbol{\lambda}} W_{\boldsymbol{\lambda}}\right)(\boldsymbol{z} ; h ; \boldsymbol{q})=\Omega_{\boldsymbol{\lambda}}(\boldsymbol{q}) \sum_{I \in \mathcal{I}_{\boldsymbol{\lambda}}} \mathcal{M}_{J}\left(\Phi_{\boldsymbol{\lambda}} W_{I}\right)(\boldsymbol{z} ; h ; \boldsymbol{q}) v_{I}
$$

Theorem 8.4. The function $\Psi_{J}(\boldsymbol{z} ; h ; \boldsymbol{q})$ is a holomorphic $\left(\mathbb{C}^{N}\right)_{\lambda}^{\otimes n}$-valued function of $\boldsymbol{z}, h, \boldsymbol{q}$ on the domain $L \subset \mathbb{C}^{n} \times \mathbb{C} \times \mathbb{C}^{N}$ such that

$$
h \notin \kappa \mathbb{Z}_{\leqslant 0}, \quad z_{a}-z_{b} \notin \kappa \mathbb{Z}, \quad z_{a}-z_{b}+h \notin \kappa \mathbb{Z},
$$

for all $a, b=1, \ldots, n, a \neq b$,

$$
\left|q_{i+1} / q_{i}\right|<1, \quad i=1, \ldots, N-1,
$$

and a branch of $\log q_{i}$ is fixed for each $i=1, \ldots, N$. Furthermore, $\Psi_{J}(\boldsymbol{z} ; h ; \boldsymbol{q})$ is a solution of the dynamical differential equations (2.3).

Proof. The weight functions $W_{I}(\boldsymbol{t} ; \boldsymbol{z} ; h)$ are polynomials in $\boldsymbol{t}, \boldsymbol{z}, h$ and do not depend on $\boldsymbol{q}$. Hence, Theorem 8.4 follows from Lemmas 8.2, 8.3, and 8.1.

Theorem 8.5. Under conditions of Theorem 8.4, the collection of $\left(\mathbb{C}^{N}\right)_{\lambda}^{\otimes n}$-valued functions $\left(\Psi_{J}(\boldsymbol{z} ; h ; \boldsymbol{q})\right)_{J \in \mathcal{I}_{\boldsymbol{\lambda}}}$ is a basis of solutions of the dynamical equations (2.3).

Proof. By formulas (8.8), (8.11), if $\left|q_{i+1} / q_{i}\right| \rightarrow 0$ for all $i=1, \ldots, N-1$, then

$$
\begin{aligned}
\Psi_{J}(\boldsymbol{z} ; h ; \boldsymbol{q}) & \simeq \Omega_{\boldsymbol{\lambda}}(\boldsymbol{q}) \operatorname{Res}_{\boldsymbol{t}=\Sigma_{J}}\left(\Phi_{\boldsymbol{\lambda}}(\boldsymbol{t} ; \boldsymbol{z} ; h ; \boldsymbol{q})\right) W_{\boldsymbol{\lambda}}\left(\Sigma_{J} ; \boldsymbol{z} ; h\right)= \\
& =\Omega_{\boldsymbol{\lambda}}(\boldsymbol{q}) \operatorname{Res}_{\boldsymbol{t}=\Sigma_{J}}\left(\Phi_{\boldsymbol{\lambda}}(\boldsymbol{t} ; \boldsymbol{z} ; h ; \boldsymbol{q})\right) \sum_{I \in \mathcal{I}_{\boldsymbol{\lambda}}} W_{I}\left(\Sigma_{J} ; \boldsymbol{z} ; h\right) v_{I}
\end{aligned}
$$

By [RTV1, Lemma 3.1], the matrix $\left(W_{I}\left(\Sigma_{J} ; \boldsymbol{z} ; h\right)\right)_{I, J \in \mathcal{I}_{\boldsymbol{\lambda}}}$ is triangular and the diagonal entries $W_{I}\left(\Sigma_{I} ; \boldsymbol{z} ; h\right)$ are nonzero if $h \neq 0$ and $z_{a}-z_{b} \neq 0, z_{a}-z_{b}+h \neq 0$, for all $a, b=1, \ldots$, $n, a \neq b$. Hence the vectors $W_{\boldsymbol{\lambda}}\left(\Sigma_{J}\right), J \in \mathcal{I}_{\boldsymbol{\lambda}}$, form a basis of $\left(\mathbb{C}^{N}\right)_{\lambda}^{\otimes n}$ and the collection $\left(\Psi_{J}(\boldsymbol{z} ; h ; \boldsymbol{q})\right)_{J \in \mathcal{I}_{\boldsymbol{\lambda}}}$ is a basis of solutions of the dynamical equations $(2.3)$. 
The functions $\Psi_{J}(\boldsymbol{z} ; h ; \boldsymbol{q})$ were considered in [TV1]. It follows from [TV1, Theorem 1.5.2], cf. [TV4], that for every $J \in \mathcal{I}_{\boldsymbol{\lambda}}$, the function $\Psi_{J}(\boldsymbol{z} ; h ; \boldsymbol{q})$ is a solution of the $q K Z$ equations (2.4).

Corollary 8.6. The collection of $\left(\mathbb{C}^{N}\right)_{\lambda}^{\otimes n}$-valued functions $\left(\Psi_{J}(\boldsymbol{z} ; h ; \boldsymbol{q})\right)_{J \in \mathcal{I}_{\boldsymbol{\lambda}}}$ is a basis of solutions of both the dynamical and $q K Z$ equations, see $(2.3),(2.4)$, with values in $\left(\mathbb{C}^{N}\right)_{\lambda}^{\otimes n}$.

Remark. The functions $\Psi_{J}(\boldsymbol{z} ; h ; \boldsymbol{q})$ are called the multidimensional q-hypergeometric solutions of the dynamical equations. In [TV5], we constructed another type of solutions of the dynamical equations called the multidimensional hypergeometric solutions.

\section{EqUIVARIANT QUANTUM DIFFERENTIAL EQUATIONS}

9.1. Partial flag varieties. Let $\boldsymbol{\lambda} \in \mathbb{Z}_{\geqslant 0}^{N},|\boldsymbol{\lambda}|=n$. Consider the partial flag variety $\mathcal{F}_{\boldsymbol{\lambda}}$ parametrizing chains of subspaces

$$
0=F_{0} \subset F_{1} \subset \ldots \subset F_{N}=\mathbb{C}^{n}
$$

with $\operatorname{dim} F_{i} / F_{i-1}=\lambda_{i}, \quad i=1, \ldots, N$. Denote by $T^{*} \mathcal{F}_{\boldsymbol{\lambda}}$ the cotangent bundle of $\mathcal{F}_{\boldsymbol{\lambda}}$ and

$$
\mathcal{X}_{n}=\bigcup_{|\boldsymbol{\lambda}|=n} T^{*} \mathcal{F}_{\boldsymbol{\lambda}}
$$

Let $u_{1}, \ldots, u_{n}$ be the standard basis of $\mathbb{C}^{n}$. For $I \in \mathcal{I}_{\boldsymbol{\lambda}}$, let $x_{I} \in \mathcal{F}_{\boldsymbol{\lambda}}$ be the point corresponding to the coordinate flag $F_{1} \subset \ldots \subset F_{N}$, where $F_{i}$ is the span of the standard basis vectors $u_{j} \in \mathbb{C}^{n}$ with $j \in I_{1} \cup \ldots \cup I_{i}$. We embed $\mathcal{F}_{\boldsymbol{\lambda}}$ in $T^{*} \mathcal{F}_{\boldsymbol{\lambda}}$ as the zero section and consider the points $x_{I}$ as points of $T^{*} \mathcal{F}_{\boldsymbol{\lambda}}$.

9.2. Equivariant cohomology. Let $A \subset G L_{n}(\mathbb{C})$ be the torus of diagonal matrices and $T=A \times \mathbb{C}^{\times}$. The group $A$ acts on $\mathbb{C}^{n}$ and hence on $T^{*} \mathcal{F}_{\boldsymbol{\lambda}}$. Let the group $\mathbb{C}^{\times}$act on $T^{*} \mathcal{F}_{\boldsymbol{\lambda}}$ by multiplication in each fiber. We denote by $-h$ its $\mathbb{C}^{\times}$-weight.

We consider the equivariant cohomology algebras $H_{T}^{*}\left(T^{*} \mathcal{F}_{\boldsymbol{\lambda}} ; \mathbb{C}\right)$ and

$$
H_{T}^{*}\left(\mathcal{X}_{n}\right)=\bigoplus_{|\lambda|=n} H_{T}^{*}\left(T^{*} \mathcal{F}_{\boldsymbol{\lambda}} ; \mathbb{C}\right)
$$

Denote by $\boldsymbol{\Gamma}_{i}=\left\{\gamma_{i, 1}, \ldots, \gamma_{i, \lambda_{i}}\right\}$ the set of the Chern roots of the bundle over $\mathcal{F}_{\boldsymbol{\lambda}}$ with fiber $F_{i} / F_{i-1}$. Let $\boldsymbol{\Gamma}=\left(\boldsymbol{\Gamma}_{1} ; \ldots ; \boldsymbol{\Gamma}_{N}\right)$. Denote by $\boldsymbol{z}=\left\{z_{1}, \ldots, z_{n}\right\}$ the Chern roots corresponding to the factors of the torus $A$. Then

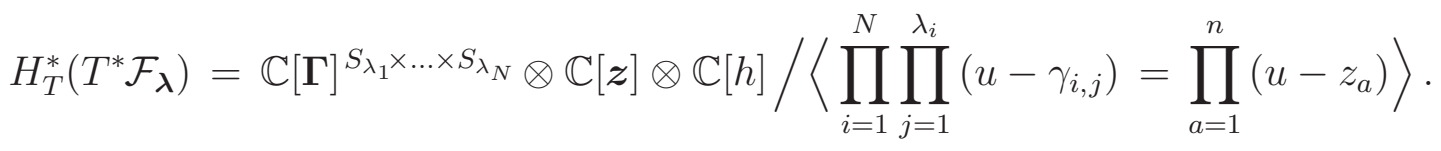

The cohomology $H_{T}^{*}\left(T^{*} \mathcal{F}_{\boldsymbol{\lambda}}\right)$ is a module over $H_{T}^{*}(p t ; \mathbb{C})=\mathbb{C}[\boldsymbol{z}] \otimes \mathbb{C}[h]$.

Notice that

$$
\prod_{i=1}^{N-1} \prod_{j=i+1}^{N} \prod_{a=1}^{\lambda_{i}} \prod_{b=1}^{\lambda_{j}}\left(\gamma_{j, b}-\gamma_{i, a}\right)\left(\gamma_{i, a}-\gamma_{j, b}-h\right)
$$


is the equivariant total Chern class of the tangent bundle of $T^{*} \mathcal{F}_{\boldsymbol{\lambda}}$ and

$$
c_{1}\left(E_{i}\right)=\sum_{a=1}^{\lambda_{i}} \gamma_{i, a}, \quad i=1, \ldots, N
$$

is the equivariant first Chern class of the vector bundle $E_{i}$ over $T^{*} \mathcal{F}_{\boldsymbol{\lambda}}$ with fiber $F_{i} / F_{i-1}$.

For $i=1, \ldots, N$, denote $\Theta_{i}=\left\{\theta_{i, 1}, \ldots, \theta_{i, \lambda^{(i)}}\right\}$ the Chern roots of the bundle $\boldsymbol{F}_{i}$ over $\mathcal{F}_{\boldsymbol{\lambda}}$ with fiber $F_{i}$. Let $\boldsymbol{\Theta}=\left(\Theta_{1}, \ldots, \Theta_{N}\right)$. The relations

$$
\prod_{a=1}^{\lambda^{(i)}}\left(u-\theta_{i, a}\right)=\prod_{j=1}^{i} \prod_{k=1}^{\lambda_{j}}\left(u-\gamma_{j, k}\right), \quad i=1, \ldots, N
$$

define the homomorphism

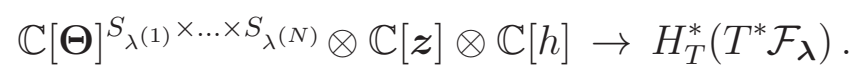

9.3. Stable envelope map. Recall the weight functions $\check{W}_{I}$ defined in Sections 3.1. Let $\check{W}_{I}(\boldsymbol{\Theta} ; \boldsymbol{z}) \in H_{T}^{*}\left(T^{*} \mathcal{F}_{\boldsymbol{\lambda}}\right)$ be the cohomology class represented by the polynomial $\check{W}_{\mathrm{id}, I}(\boldsymbol{t} ; \boldsymbol{z})$ with the variables $t_{a}^{(i)}$ replaced by $\theta_{i, a}$ for all $i=1, \ldots, N-1, a=1, \ldots, \lambda^{(i)}$. Denote

$$
c_{\boldsymbol{\lambda}}(\boldsymbol{\Theta})=\prod_{i=1}^{N-1} \prod_{a=1}^{\lambda^{(i)}} \prod_{b=1}^{\lambda^{(i)}}\left(\theta_{i, a}-\theta_{i, b}-h\right) \in H_{T}^{*}\left(T^{*} \mathcal{F}_{\boldsymbol{\lambda}}\right)
$$

Observe that $c_{\boldsymbol{\lambda}}(\boldsymbol{\Theta})$ is the equivariant Euler class of the bundle $\bigoplus_{a=1}^{N-1} \operatorname{Hom}\left(\boldsymbol{F}_{a}, \boldsymbol{F}_{a}\right)$ if we make $\mathbb{C}^{\times}$act on it with weight $-h$.

Theorem 9.1 ([RTV1, Theorem 4.1]). For any $\boldsymbol{\lambda}$ and any $I \in \mathcal{I}_{\boldsymbol{\lambda}}$, the cohomology class $\check{W}_{I}(\boldsymbol{\Theta} ; \boldsymbol{z}) \in H_{T}^{*}\left(T^{*} \mathcal{F}_{\boldsymbol{\lambda}}\right)$ is divisible by $c_{\boldsymbol{\lambda}}(\boldsymbol{\Theta})$, that is, there exists a unique element $\mathrm{Stab}_{I} \in$ $H_{T}^{*}\left(T^{*} \mathcal{F}_{\boldsymbol{\lambda}}\right)$ such that

$$
\left[\check{W}_{I}(\boldsymbol{\Theta} ; \boldsymbol{z})\right]=c_{\boldsymbol{\lambda}}(\boldsymbol{\Theta}) \cdot \mathrm{Stab}_{I}
$$

Define the stable envelope map by the rule

$$
\text { Stab }:\left(\mathbb{C}^{N}\right)^{\otimes n} \otimes \mathbb{C}[\boldsymbol{z}] \otimes \mathbb{C}[h] \rightarrow H_{T}^{*}\left(\mathcal{X}_{n}\right), \quad v_{I} \mapsto \operatorname{Stab}_{I}
$$

Remark. Stable envelope maps for Nakajima quiver varieties were introduced in [MO]. They were defined there geometrically in terms of the associated torus action. The map Stab given by formula (9.6) is the stable envelope map of [MO] for the Nakajima quiver variety $\mathcal{X}_{n}$, described in terms of the Chern roots $\Theta, \boldsymbol{z}, h$, see [RTV1].

Remark. After the substitution $h=1$ the classes $\operatorname{Stab}_{I} \in H_{T}^{*}\left(T^{*} \mathcal{F}_{\boldsymbol{\lambda}}\right)$ can be considered as elements of the equivariant cohomology $H_{\left(\mathbb{C}^{\times}\right)^{n}}^{*}\left(\mathcal{F}_{\boldsymbol{\lambda}}\right)$ of the partial flag variety $\mathcal{F}_{\boldsymbol{\lambda}}$ (and not of the cotangent bundle $T^{*} \mathcal{F}_{\boldsymbol{\lambda}}$ ). These new classes are the equivariant Chern-SchwartzMacPherson classes (CSM classes) of the corresponding Schubert cells, see [RV]. 
Let $\mathbb{C}(\boldsymbol{z} ; h)$ be the algebra of rational functions in $\boldsymbol{z}, h$. The map

$$
\text { Stab : }\left(\mathbb{C}^{N}\right)^{\otimes n} \otimes \mathbb{C}(\boldsymbol{z} ; h) \rightarrow H_{T}^{*}\left(\mathcal{X}_{n}\right) \otimes \mathbb{C}(\boldsymbol{z} ; h), \quad v_{I} \mapsto \operatorname{Stab}_{I},
$$

is an isomorphism of $\mathbb{C}(\boldsymbol{z} ; h)$-modules by [RTV1, Lemma 6.7].

9.4. $H_{T}^{*}\left(T^{*} \mathcal{F}_{\boldsymbol{\lambda}}\right)$-valued weight function. Define the $H_{T}^{*}\left(T^{*} \mathcal{F}_{\boldsymbol{\lambda}}\right)$-valued function $\widehat{W}(\boldsymbol{t} ; \boldsymbol{\Gamma})$ as follows:

$$
\begin{gathered}
\widehat{W}(\boldsymbol{t} ; \boldsymbol{\Gamma})=\operatorname{Sym}_{t_{1}^{(1)}, \ldots, t_{\lambda(1)}^{(1)}} \ldots \operatorname{Sym}_{t_{1}^{(N-1)}, \ldots, t_{\lambda\left({ }^{(N-1)}\right.}^{(N-1)}} \widehat{U}(\boldsymbol{t} ; \boldsymbol{\Gamma}), \\
\widehat{U}(\boldsymbol{t} ; \boldsymbol{\Gamma})=\prod_{j=1}^{N-1} \prod_{a=1}^{\lambda^{(j)}}\left(\prod_{c=1}^{a-1}\left(t_{a}^{(j)}-t_{c}^{(j+1)}-h\right) \prod_{d=a+1}^{\lambda^{(j+1)}}\left(t_{a}^{(j)}-t_{d}^{(j+1)}\right) \prod_{b=a+1}^{\lambda^{(j)}} \frac{t_{a}^{(j)}-t_{b}^{(j)}-h}{t_{a}^{(j)}-t_{b}^{(j)}}\right) .
\end{gathered}
$$

where $\left(t_{1}^{(N)}, \ldots, t_{n}^{(N)}\right)=\left(\gamma_{1,1}, \ldots, \gamma_{1, \lambda_{1}}, \gamma_{2,1}, \ldots, \gamma_{2, \lambda_{2}}, \ldots, \gamma_{N, 1}, \ldots, \gamma_{N, \lambda_{N}}\right)$, cf. formula (3.1) for $I=I^{\min }=\left(\left\{1, \ldots, \lambda_{1}\right\}, \ldots,\left\{n-\lambda_{N}+1, \ldots, n\right\}\right)$.

Example. Let $N=2, \boldsymbol{\lambda}=(1, n-1)$. Then $\widehat{W}(\boldsymbol{t} ; \boldsymbol{\Gamma})=\prod_{a=1}^{n-1}\left(t_{1}^{(1)}-\gamma_{2, a}\right)$.

Let $N=3, \boldsymbol{\lambda}=(1,1,1)$. Then

$$
\begin{aligned}
\widehat{W}(\boldsymbol{t} ; \boldsymbol{\Gamma}) & =\left(t_{1}^{(1)}-t_{2}^{(2)}\right)\left(t_{1}^{(2)}-\gamma_{2,1}\right)\left(t_{1}^{(2)}-\gamma_{3,1}\right)\left(t_{2}^{(2)}-\gamma_{1,1}-h\right)\left(t_{2}^{(2)}-\gamma_{3,1}\right) \frac{t_{1}^{(2)}-t_{2}^{(2)}-h}{t_{1}^{(2)}-t_{2}^{(2)}}+ \\
& +\left(t_{1}^{(1)}-t_{1}^{(2)}\right)\left(t_{2}^{(2)}-\gamma_{2,1}\right)\left(t_{2}^{(2)}-\gamma_{3,1}\right)\left(t_{1}^{(2)}-\gamma_{1,1}-h\right)\left(t_{1}^{(2)}-\gamma_{3,1}\right) \frac{t_{2}^{(2)}-t_{1}^{(2)}-h}{t_{2}^{(2)}-t_{1}^{(2)}} .
\end{aligned}
$$

Define

$$
Q(\boldsymbol{\Gamma})=\prod_{i=1}^{N-1} \prod_{j=i+1}^{N} \prod_{a=1}^{\lambda_{i}} \prod_{b=1}^{\lambda_{j}}\left(\gamma_{i, a}-\gamma_{j, b}-h\right) \in H_{T}^{*}\left(T^{*} \mathcal{F}_{\boldsymbol{\lambda}}\right)
$$

The image of the $\left(\mathbb{C}^{N}\right)_{\lambda}^{\otimes n}$-valued weight function $W_{\boldsymbol{\lambda}}(\boldsymbol{t} ; \boldsymbol{z})$, see $(7.4)$, is given by the next proposition.

Proposition 9.2. We have

$$
\sum_{I \in \mathcal{I}_{\boldsymbol{\lambda}}} W_{I}(\boldsymbol{t} ; \boldsymbol{z}) \operatorname{Stab}_{\mathrm{id}, I}=Q(\boldsymbol{\Gamma}) \widehat{W}(\boldsymbol{t} ; \boldsymbol{\Gamma})
$$

Proof. Recall that $W_{I}(\boldsymbol{t} ; \boldsymbol{z})=(-h)^{-\lambda\{1\}} \check{W}_{\sigma_{0}, I}(\boldsymbol{t} ; \boldsymbol{z})$, see (3.4). Recall the discrete cycle $\Sigma_{J}$ given by (8.4). Let $\sigma^{I} \in S_{n}$ be a permutation such that $\sigma^{I}\left(I^{\min }\right)=I$. Then formula (9.10) is equivalent to the following equality

$$
\sum_{I \in \mathcal{I}_{\boldsymbol{\lambda}}} \check{W}_{\sigma_{0}, I}(\boldsymbol{t} ; \boldsymbol{z}) \check{W}_{I}\left(\Sigma_{J} ; \boldsymbol{z}\right)=c_{\boldsymbol{\lambda}}\left(\Sigma_{J}\right) Q\left(\boldsymbol{z}_{J}\right) \check{W}_{\sigma^{J}, J}(\boldsymbol{t} ; \boldsymbol{z}) \text {. }
$$

For the proof of formula (9.11), consider the function

$$
Z(\boldsymbol{t} ; \tilde{\boldsymbol{t}} ; \boldsymbol{z})=\sum_{I \in \mathcal{I}_{\boldsymbol{\lambda}}} \check{W}_{\sigma_{0}, I}(\boldsymbol{t} ; \boldsymbol{z}) \check{W}_{I}(\tilde{\boldsymbol{t}} ; \boldsymbol{z})
$$


Here $\tilde{\boldsymbol{t}}$ is an additional set of variables similar to $\boldsymbol{t}$. Then formula (9.11) reads

$$
Z\left(\boldsymbol{t} ; \Sigma_{J} ; \boldsymbol{z}\right)=c_{\boldsymbol{\lambda}}\left(\Sigma_{J}\right) Q\left(\boldsymbol{z}_{J}\right) \check{W}_{\sigma^{J}, J}(\boldsymbol{t} ; \boldsymbol{z}) .
$$

Three-term relations (3.3) imply that for any $\sigma \in S_{n}$, we have

$$
Z(\boldsymbol{t} ; \tilde{\boldsymbol{t}} ; \boldsymbol{z})=\sum_{I \in \mathcal{I}_{\boldsymbol{\lambda}}} \check{W}_{\sigma, I}(\boldsymbol{t} ; \boldsymbol{z}) \check{W}_{\sigma \sigma_{0}, I}(\tilde{\boldsymbol{t}} ; \boldsymbol{z})
$$

By [RTV1, Lemma 3.2], we have $\check{W}_{\sigma^{J} \sigma_{0}, I}\left(\Sigma_{J}, \boldsymbol{z}\right)=c_{\boldsymbol{\lambda}}\left(z_{J}\right) Q\left(\boldsymbol{z}_{J}\right) \delta_{I, J}$. Thus taking $\sigma=\sigma^{J}$, $\tilde{\boldsymbol{t}}=\Sigma_{J}$ in formula (9.13), we get equality (9.12). Proposition 9.2 is proved.

Define the cohomology classes

$$
R(\boldsymbol{\Gamma})=\prod_{i=1}^{N-1} \prod_{j=i+1}^{N} \prod_{a=1}^{\lambda_{i}} \prod_{b=1}^{\lambda_{j}}\left(\gamma_{i, a}-\gamma_{j, b}\right)
$$

and

$$
R_{I}(\boldsymbol{\Gamma} ; \boldsymbol{z})=\prod_{i=1}^{N-1} \prod_{j=i+1}^{N} \prod_{a=1}^{\lambda_{i}} \prod_{b \in I_{j}}\left(\gamma_{i, a}-z_{b}\right), \quad I \in \mathcal{I}_{\boldsymbol{\lambda}} .
$$

Notice that $R_{I}\left(\boldsymbol{z}_{J} ; \boldsymbol{z}\right)=R\left(\boldsymbol{z}_{J}\right) \delta_{I, J}$.

Proposition 9.3. For any $K \in \mathcal{I}_{\boldsymbol{\lambda}}$, we have

$$
\sum_{I \in \mathcal{I}_{\boldsymbol{\lambda}}} W_{I}\left(\Sigma_{K} ; \boldsymbol{z}\right) \operatorname{Stab}_{\mathrm{id}, I}=(-h)^{-\lambda^{\{1\}}} c_{\boldsymbol{\lambda}}(\boldsymbol{\Theta}) R_{K}(\boldsymbol{\Gamma} ; \boldsymbol{z}) Q(\boldsymbol{\Gamma}) .
$$

Proof. Formula (9.16) is equivalent to the equality

$$
\sum_{I \in \mathcal{I}_{\boldsymbol{\lambda}}} \check{W}_{\sigma_{0}, I}\left(\Sigma_{K} ; \boldsymbol{z}\right) \check{W}_{I}\left(\Sigma_{J} ; \boldsymbol{z}\right)=\left(c_{\boldsymbol{\lambda}}\left(\Sigma_{J}\right)\right)^{2} R\left(\boldsymbol{z}_{J}\right) Q\left(\boldsymbol{z}_{J}\right) \delta_{J, K}
$$

By [RTV1, Lemma 3.2], we have $\check{W}_{\sigma^{J}, J}\left(\Sigma_{K}, \boldsymbol{z} ; h\right)=c_{\boldsymbol{\lambda}}\left(z_{J}\right) R\left(\boldsymbol{z}_{J}\right) \delta_{J, K}$. Thus taking $\tilde{\boldsymbol{t}}=\Sigma_{K}$ in formula (9.11), we get equality (9.17).

Formula (9.17) also follows from [RTV1, Lemma 3.4]. Proposition 9.3 is proved.

9.5. Quantum multiplication by divisors on $H_{T}^{*}\left(T^{*} \mathcal{F}_{\boldsymbol{\lambda}}\right)$. The quantum multiplication by divisors on $H_{T}^{*}\left(T^{*} \mathcal{F}_{\boldsymbol{\lambda}}\right)$ is described in [MO]. The fundamental equivariant cohomology classes of divisors on $T^{*} \mathcal{F}_{\boldsymbol{\lambda}}$ are linear combinations of $D_{i}=\gamma_{i, 1}+\ldots+\gamma_{i, \lambda_{i}}, i=1, \ldots, N$. The quantum multiplication $D_{i} *_{\tilde{q}}: H_{T}^{*}\left(T^{*} \mathcal{F}_{\boldsymbol{\lambda}}\right) \rightarrow H_{T}^{*}\left(T^{*} \mathcal{F}_{\boldsymbol{\lambda}}\right)$ by the divisor $D_{i}$ depends on parameters $\tilde{\boldsymbol{q}}=\left(\tilde{q}_{1}, \ldots, \tilde{q}_{N}\right) \in\left(\mathbb{C}^{\times}\right)^{N}$ and is given in [MO, Theorem 10.2.1].

The quantum connection $\nabla_{\boldsymbol{\lambda}, \tilde{q}, \tilde{\kappa}}^{\text {quant }}$ on $H_{T}^{*}\left(T^{*} \mathcal{F}_{\boldsymbol{\lambda}}\right)$ is defined by the formula

$$
\nabla_{\boldsymbol{\lambda}, \tilde{\boldsymbol{q}}, \tilde{\kappa}, i}^{\text {quant }}=\tilde{\kappa} \tilde{q}_{i} \frac{\partial}{\partial \tilde{q}_{i}}-D_{i} * \tilde{\boldsymbol{q}}, \quad i=1, \ldots, N
$$


where $\tilde{\kappa} \in \mathbb{C}^{\times}$is the parameter of the connection, see [BMO]. The system of equations for flat sections of the quantum connection is called the system of the equivariant quantum differential equations.

The isomorphism Stab allows us to compare the operators $\nabla_{\boldsymbol{\lambda}, \boldsymbol{q}, \kappa, i}:=\left.\nabla_{\boldsymbol{q}, \kappa, i}\right|_{\left(\mathbb{C}^{N}\right)_{\boldsymbol{\lambda}}^{\otimes n}}$ of the dynamical connection on $\left(\mathbb{C}^{N}\right)_{\boldsymbol{\lambda}}^{\otimes n}$, see $(2.2)$, and the operators $\nabla_{\boldsymbol{\lambda}, \tilde{\boldsymbol{q}}, \tilde{\kappa}, i}^{\text {quant }}$ of the quantum connection on $H_{T}^{*}\left(T^{*} \mathcal{F}_{\boldsymbol{\lambda}}\right)$.

Recall the dynamical Hamiltonians $X_{i}(\boldsymbol{z} ; h ; \boldsymbol{q})$, see $(2.1)$. Define the modified dynamical Hamiltonians

$$
\begin{aligned}
& X_{\boldsymbol{\lambda}, i}^{-}(\boldsymbol{z} ; h ; \boldsymbol{q})= \\
& =\left.X_{i}(\boldsymbol{z} ; h ; \boldsymbol{q})\right|_{\left(\mathbb{C}^{N}\right)_{\lambda}^{\otimes n}}-h \sum_{j=1}^{i-1} \frac{q_{i}}{q_{i}-q_{j}} \min \left(\lambda_{i}, \lambda_{j}\right)-h \sum_{j=i+1}^{N} \frac{q_{j}}{q_{i}-q_{j}} \min \left(\lambda_{i}, \lambda_{j}\right) .
\end{aligned}
$$

The modified dynamical connection on $\left(\mathbb{C}^{N}\right)_{\lambda}^{\otimes n}$ is

$$
\nabla_{\boldsymbol{\lambda}, \boldsymbol{q}, \kappa, i}^{-}=\kappa q_{i} \frac{\partial}{\partial q_{i}}-X_{\boldsymbol{\lambda}, i}^{-}(\boldsymbol{z} ; h ; \boldsymbol{q}), \quad i=1, \ldots, N
$$

see [GRTV, Section 3.4]. Recall that $h_{\mathrm{GRTV}}=-h$.

Theorem 9.4 ([RTV1, Corollary 7.6]). The isomorphism Stab identifies the operators $D_{i} *_{\tilde{q}}$ of quantum multiplication by $D_{i}$ on $H_{T}^{*}\left(T^{*} \mathcal{F}_{\boldsymbol{\lambda}}\right)$ with the action of the modified dynamical Hamiltonians $X_{\boldsymbol{\lambda}, i}^{-}\left(\boldsymbol{z} ; h ; \tilde{\boldsymbol{q}}^{-1}\right)$ on $\left(\mathbb{C}^{N}\right)_{\boldsymbol{\lambda}}^{\otimes n}$, where $\tilde{\boldsymbol{q}}^{-1}=\left(\tilde{q}_{1}^{-1}, \ldots, \tilde{q}_{N}^{-1}\right)$. Consequently, the differential operators $\nabla_{\boldsymbol{\lambda}, \tilde{\boldsymbol{q}}, \tilde{\kappa}, i}^{\text {quant }}$ are identified with the differential operators $\nabla_{\boldsymbol{\lambda}, \tilde{\boldsymbol{q}}^{-1,-\tilde{\kappa}, i}}$.

See also [RTV1, Theorem 7.5].

Set

$$
\widehat{\Omega}_{\boldsymbol{\lambda}}(\tilde{\boldsymbol{q}} ; \tilde{\kappa})=\prod_{i=1}^{N-1} \prod_{j=i+1}^{N}\left(1-\tilde{q}_{i} / \tilde{q}_{j}\right)^{h \min \left(0, \lambda_{j}-\lambda_{i}\right) / \tilde{\kappa}} .
$$

Set $\lambda_{\{2\}}=\sum_{1 \leqslant i<j \leqslant N} \lambda_{i} \lambda_{j}$. For any $I \in \mathcal{I}_{\boldsymbol{\lambda}}$, define

$$
\begin{array}{r}
\widehat{\Psi}_{I}(\boldsymbol{z} ; h ; \tilde{\boldsymbol{q}} ; \tilde{\kappa})=\tilde{\kappa}^{-\lambda^{\{1\}}-2 \lambda_{\{2\}}}(-1)^{\lambda^{\{1\}}+\lambda_{\{2\}}}(\Gamma(-h / \tilde{\kappa}))^{-\lambda^{\{1\}}} \widetilde{\Psi}_{I}(\boldsymbol{z} ; h ; \tilde{\boldsymbol{q}} ; \tilde{\kappa}), \\
\widetilde{\Psi}_{I}(\boldsymbol{z} ; h ; \tilde{\boldsymbol{q}} ; \tilde{\kappa})=\widehat{\Omega}_{\boldsymbol{\lambda}}(\tilde{\boldsymbol{q}} ; \tilde{\kappa}) \sum_{\boldsymbol{r} \in \mathbb{Z}_{\geqslant 0}^{\lambda\{1\}}} \operatorname{Res}_{\boldsymbol{t}=\Sigma_{I}+\boldsymbol{r} \tilde{\kappa}}\left(\Phi_{\boldsymbol{\lambda}}\left(\boldsymbol{t} ; \boldsymbol{z} ; \tilde{\boldsymbol{q}}^{-1} ;-\tilde{\kappa}\right)\right) Q(\boldsymbol{\Gamma}) \widehat{W}\left(\Sigma_{I}+\boldsymbol{r} \tilde{\kappa} ; \boldsymbol{\Gamma}\right) .
\end{array}
$$

For $\tilde{\boldsymbol{q}}, \tilde{\kappa}$ given, $\widehat{\Psi}_{I}(\boldsymbol{z} ; h ; \tilde{\boldsymbol{q}} ; \tilde{\kappa})$ belongs to the extension of $H_{T}^{*}\left(T^{*} \mathcal{F}_{\boldsymbol{\lambda}}\right)$ by holomorphic functions in $\boldsymbol{z}, h$ on the domain $L^{\prime} \subset \mathbb{C}^{n} \times \mathbb{C}$ such that

$$
h \notin \tilde{\kappa} \mathbb{Z}_{\geqslant 0}, \quad z_{a}-z_{b} \notin \tilde{\kappa} \mathbb{Z}, \quad z_{a}-z_{b}+h \notin \tilde{\kappa} \mathbb{Z},
$$

for all $a, b=1, \ldots, n, a \neq b$. Furthermore, $\widehat{\Psi}_{I}(\boldsymbol{z} ; h ; \tilde{\boldsymbol{q}} ; \tilde{\kappa})$ is a holomorphic function of $\tilde{\boldsymbol{q}}$ on the domain $L^{\prime \prime} \subset \mathbb{C}^{N}$ such that $\left|\tilde{q}_{i} / \tilde{q}_{i+1}\right|<1, i=1, \ldots, N-1$, and a branch of $\log \tilde{q}_{i}$ is fixed for each $i=1, \ldots, N$. 
Example. Let $N=2, n=2, \boldsymbol{\lambda}=(1,1)$. Recall the Gauss hypergeometric series

$$
{ }_{2} F_{1}(a, b ; c ; x)=\sum_{m=0}^{\infty} \frac{(a)_{m}(b)_{m}}{(c)_{m}} \frac{x^{m}}{m !},
$$

where $(u)_{m}=u(u-1) \ldots(u-m+1)$. Set

and

$$
F\left(z_{1}, z_{2} ; h ; \tilde{\kappa} ; x\right)={ }_{2} F_{1}\left(-\frac{h}{\tilde{\kappa}}, \frac{z_{1}-z_{2}-h}{\tilde{\kappa}} ; 1+\frac{z_{1}-z_{2}}{\tilde{\kappa}} ; x\right)
$$

Then

$$
F^{\prime}\left(z_{1}, z_{2} ; h ; \tilde{\kappa} ; x\right)=\frac{\partial F}{\partial x}\left(z_{1}, z_{2} ; h ; \tilde{\kappa} ; x\right) .
$$

$$
\begin{aligned}
& \widehat{\Psi}_{(\{1\},\{2\})}\left(z_{1}, z_{2} ; h ; \tilde{q}_{1}, \tilde{q}_{2} ; \tilde{\kappa}\right)= \\
& =\tilde{\kappa}^{-2}\left(e^{-\pi \sqrt{-1}} \tilde{q}_{1}\right)^{z_{1} / \tilde{\kappa}}\left(e^{-\pi \sqrt{-1}} \tilde{q}_{2}\right)^{z_{2} / \tilde{\kappa}} \Gamma\left(\frac{z_{2}-z_{1}}{\tilde{\kappa}}\right) \Gamma\left(\frac{z_{1}-z_{2}-h}{\tilde{\kappa}}\right) \\
& \quad \times\left(\gamma_{1,1}-\gamma_{2,1}-h\right)\left(\left(\gamma_{2,1}-z_{1}\right) F\left(z_{1}, z_{2} ; h ; \tilde{\kappa} ; \tilde{q}_{1} / \tilde{q}_{2}\right)-\tilde{\kappa}\left(\tilde{q}_{1} / \tilde{q}_{2}\right) F^{\prime}\left(z_{1}, z_{2} ; h ; \tilde{\kappa} ; \tilde{q}_{1} / \tilde{q}_{2}\right)\right)
\end{aligned}
$$

and $\widehat{\Psi}_{(\{2\},\{1\})}\left(z_{1}, z_{2} ; h ; \tilde{q}_{1}, \tilde{q}_{2} ; \widetilde{\kappa}\right)=\widehat{\Psi}_{(\{1\},\{2\})}\left(z_{2}, z_{1} ; h ; \tilde{q}_{1}, \tilde{q}_{2} ; \tilde{\kappa}\right)$.

Theorem 9.5. The collection of functions $\left(\widehat{\Psi}_{I}(\boldsymbol{z} ; h ; \tilde{\boldsymbol{q}} ; \tilde{\kappa})\right)_{I \in \mathcal{I}_{\boldsymbol{\lambda}}}$ is a basis of solutions of both the quantum differential equations $\nabla_{\boldsymbol{\lambda}, \tilde{\boldsymbol{q}}, \tilde{\kappa}, i}^{\text {quant }} f=0, i=1, \ldots, N$, and the associated $q K Z$ difference equations.

Proof. The statement follows from Theorems 8.4, 8.5, 9.4, and Proposition 9.2, see Corollary 8.6.

Remark. The integral representations for solutions of the equivariant quantum differential equations is a manifestation of a version of mirror symmetry. The basis of solutions given by Theorem 9.5 is an analog of Givental's J-function.

For $\tilde{q}_{i} / \tilde{q}_{i+1} \rightarrow 0$ for all $i=1, \ldots, N-1$, the leading term of the asymptotics of $\widehat{\Psi}_{I}(\boldsymbol{z} ; h ; \tilde{\boldsymbol{q}} ; \tilde{\kappa})$ is given by taking the residue at $\boldsymbol{t}=\Sigma_{I}$.

Theorem 9.6. Assume that $\tilde{q}_{i} / \tilde{q}_{i+1} \rightarrow 0$ for all $i=1, \ldots, N-1$. Then

$$
\begin{aligned}
& \widehat{\Psi}_{I}(\boldsymbol{z} ; h ; \tilde{\boldsymbol{q}} ; \tilde{\kappa})=\prod_{i=1}^{N}\left(e^{\pi \sqrt{-1}\left(\lambda_{i}-n\right)} \tilde{q}_{i}\right)^{\sum_{a \in I_{i}} z_{a} / \tilde{\kappa}} \\
& \times \prod_{i=1}^{N-1} \prod_{j=i+1}^{N} \prod_{a \in I_{i}} \prod_{b \in I_{j}} \Gamma\left(1+\frac{z_{b}-z_{a}}{\tilde{\kappa}}\right) \Gamma\left(1+\frac{z_{a}-z_{b}-h}{\tilde{\kappa}}\right) \\
& \times\left(\Delta_{I}+\sum_{\substack{\boldsymbol{m} \in \mathbb{Z}_{\geqslant 0}^{N-1} \\
\boldsymbol{m} \neq 0}} \widehat{\Psi}_{I, \boldsymbol{m}}(\boldsymbol{z} ; h ; \tilde{\kappa}) \prod_{i=1}^{N-1}\left(\frac{\tilde{q}_{i}}{\tilde{q}_{i+1}}\right)^{m_{i}}\right),
\end{aligned}
$$


where $\Delta_{I}(\boldsymbol{\Gamma}, \boldsymbol{z})=R_{I}(\boldsymbol{\Gamma} ; \boldsymbol{z}) / R\left(\boldsymbol{z}_{I}\right)$ is the cohomology class such that $\Delta_{I}\left(\boldsymbol{z}_{J} ; \boldsymbol{z}\right)=\delta_{I, J}$, and the classes $\widehat{\Psi}_{I, \boldsymbol{m}}(\boldsymbol{z} ; h ; \tilde{\kappa})$ are rational functions in $\boldsymbol{z}, h, \tilde{\kappa}$, regular on the domain $h \notin \widetilde{\kappa} \mathbb{Z}_{\geqslant 0}$, $z_{a}-z_{b} \notin \tilde{\kappa} \mathbb{Z}, z_{a}-z_{b}+h \notin \tilde{\kappa} \mathbb{Z}$, for all $a, b=1, \ldots, n, a \neq b$.

Proof. The statement follows from formula (9.21) and Propositions 9.2, 9.3.

Example. Let $N=2, n=2, \boldsymbol{\lambda}=(1,1)$. As $\tilde{q}_{1} / \tilde{q}_{2} \rightarrow 0$, the leading term of the solution $\widehat{\Psi}_{(\{1\},\{2\})}\left(z_{1}, z_{2} ; h ; \tilde{q}_{1}, \tilde{q}_{2} ; \tilde{\kappa}\right)$ is the cohomology class

$$
\left(e^{-\pi \sqrt{-1}} \tilde{q}_{1}\right)^{z_{1} / \tilde{\kappa}}\left(e^{-\pi \sqrt{-1}} \tilde{q}_{2}\right)^{z_{2} / \tilde{\kappa}} \Gamma\left(1+\frac{z_{2}-z_{1}}{\tilde{\kappa}}\right) \Gamma\left(1+\frac{z_{1}-z_{2}-h}{\tilde{\kappa}}\right) \Delta_{(\{1\},\{2\})}
$$

and the leading term of the solution $\widehat{\Psi}_{(\{2\},\{1\})}\left(z_{1}, z_{2} ; h ; \tilde{q}_{1}, \tilde{q}_{2} ; \tilde{\kappa}\right)$ is the cohomology class

$$
\left(e^{-\pi \sqrt{-1}} \tilde{q}_{1}\right)^{z_{2} / \tilde{\kappa}}\left(e^{-\pi \sqrt{-1}} \tilde{q}_{2}\right)^{z_{1} / \tilde{\kappa}} \Gamma\left(1+\frac{z_{1}-z_{2}}{\tilde{\kappa}}\right) \Gamma\left(1+\frac{z_{2}-z_{1}-h}{\tilde{\kappa}}\right) \Delta_{(\{2\},\{1\})} .
$$

\section{Quantum Pieri Rules}

10.1. Quantum equivariant cohomology algebra $\mathcal{H}_{T}^{\tilde{q}}\left(T^{*} \mathcal{F}_{\boldsymbol{\lambda}}\right)$. Let $\tilde{\boldsymbol{q}}=\left(\tilde{q}_{1}, \ldots, \tilde{q}_{N}\right) \in$ $\left(\mathbb{C}^{\times}\right)^{N}$ have distinct coordinates. The quantum equivariant cohomology algebra $\mathcal{H}_{T}^{\tilde{q}}\left(T^{*} \mathcal{F}_{\boldsymbol{\lambda}}\right)$ is the algebra generated by the operators $D_{i} * \tilde{q}: H_{T}^{*}\left(T^{*} \mathcal{F}_{\boldsymbol{\lambda}}\right) \rightarrow H_{T}^{*}\left(T^{*} \mathcal{F}_{\boldsymbol{\lambda}}\right)$ of quantum multiplication by the divisors $D_{i}, i=1, \ldots, N$, see details in [MO, GRTV]. The algebra can be defined by generators and relations as follows.

Introduce the variables $\tilde{\gamma}_{i, 1}, \ldots, \tilde{\gamma}_{i, \lambda_{i}}$ for $i=1, \ldots, N$. Set

$$
W^{\tilde{q}}(u)=\operatorname{det}\left(\tilde{q}_{i}^{j-1} \prod_{k=1}^{\lambda_{i}}\left(u-\tilde{\gamma}_{i, k}-h(i-j)\right)\right)_{i, j=1}^{N} .
$$

Theorem 10.1. The quantum equivariant cohomology algebra $\mathcal{H}_{T}^{\tilde{q}}\left(T^{*} \mathcal{F}_{\boldsymbol{\lambda}}\right)$ is isomorphic to the algebra

$$
\mathbb{C}[\widetilde{\boldsymbol{\Gamma}}]^{S_{\lambda_{1}} \times \ldots \times S_{\lambda_{N}}} \otimes \mathbb{C}[\boldsymbol{z}] \otimes \mathbb{C}[h] /\left\langle W^{\tilde{q}}(u)=\prod_{1 \leqslant i<j \leqslant N}\left(\tilde{q}_{j}-\tilde{q}_{i}\right) \prod_{a=1}^{n}\left(u-z_{a}\right)\right\rangle
$$

where $\widetilde{\boldsymbol{\Gamma}}=\left(\tilde{\gamma}_{1,1}, \ldots, \tilde{\gamma}_{1, \lambda_{1}}, \ldots, \tilde{\gamma}_{N, 1}, \ldots, \tilde{\gamma}_{N, \lambda_{N}}\right)$, and the correspondence is

$$
D_{i} * \tilde{q} \mapsto\left[\sum_{k=1}^{\lambda_{i}} \tilde{\gamma}_{i, k}-h \sum_{j=1}^{i-1} \frac{\tilde{q}_{j}}{\tilde{q}_{j}-\tilde{q}_{i}} \min \left(0, \lambda_{j}-\lambda_{i}\right)-h \sum_{j=i+1}^{N} \frac{\tilde{q}_{i}}{\tilde{q}_{j}-\tilde{q}_{i}} \min \left(0, \lambda_{j}-\lambda_{i}\right)\right] .
$$

This theorem follows from [GRTV, Theorems 6.5, 7.10, and Lemma 6.10], see also [MTV2]. Notice that the parameters in this paper and in [GRTV] are related as follows: $h=-h_{\mathrm{GRTV}}$, $\tilde{q}_{i}=q_{i}^{-1}, i=1, \ldots, N$.

Example. Let $N=2, n=2, \boldsymbol{\lambda}=(1,1)$. Then $D_{i} *_{\tilde{\boldsymbol{q}}} \mapsto \tilde{\gamma}_{i, 1}, i=1,2$, and the relations are

$$
\tilde{\gamma}_{1,1}+\tilde{\gamma}_{2,1}=z_{1}+z_{2}, \quad \tilde{\gamma}_{1,1} \tilde{\gamma}_{2,1}-h \frac{\tilde{q}_{1}}{\tilde{q}_{2}-\tilde{q}_{1}}\left(\tilde{\gamma}_{1,1}-\tilde{\gamma}_{2,1}-h\right)=z_{1} z_{2}
$$


It is easy to see that the algebra $\mathcal{H}_{T}^{\tilde{q}}\left(T^{*} \mathcal{F}_{\lambda}\right)$ does not change if all $\tilde{q}_{1}, \ldots, \tilde{q}_{N}$ are multiplied by the same number. In the limit $\tilde{q}_{i} / \tilde{q}_{i+1} \rightarrow 0, i=1, \ldots, N-1$, the relations in $\mathcal{H}_{T}^{\tilde{q}}\left(T^{*} \mathcal{F}_{\boldsymbol{\lambda}}\right)$ turn into the relations in $H_{T}^{*}\left(T^{*} \mathcal{F}_{\boldsymbol{\lambda}}\right)$, see formula (9.1).

10.2. Quantum equivariant Pieri rules. Recall the weight functions $W_{I}(\boldsymbol{t} ; \boldsymbol{z})$, see (3.5). Introduce the variables $\widetilde{\boldsymbol{\Theta}}_{i}=\left\{\tilde{\theta}_{i, 1}, \ldots, \tilde{\theta}_{i, \lambda^{(i)}}\right\}, \widetilde{\boldsymbol{\Theta}}=\left(\widetilde{\boldsymbol{\Theta}}_{1}, \ldots, \widetilde{\boldsymbol{\Theta}}_{N}\right)$. Let $W_{I}(\widetilde{\boldsymbol{\Theta}} ; \boldsymbol{z})$ be the polynomial $W_{I}(\boldsymbol{t} ; \boldsymbol{z})$ with the variables $t_{a}^{(i)}$ replaced by $\tilde{\theta}_{i, a}$ for all $i=1, \ldots, N-1, a=1$, $\ldots, \lambda^{(i)}$. For any $m=1, \ldots, N-1$, the relation

$$
\operatorname{det}\left(\tilde{q}_{i}^{j-1} \prod_{k=1}^{\lambda_{i}}\left(u-\tilde{\gamma}_{i, k}-h(i-j)\right)\right)_{i, j=1}^{m}=\prod_{1 \leqslant i<j \leqslant m}\left(\tilde{q}_{j}-\tilde{q}_{i}\right) \prod_{a=1}^{\lambda^{(m)}}\left(u-\tilde{\theta}_{m, a}\right)
$$

allows us to express the elementary symmetric functions in the variables $\widetilde{\boldsymbol{\Theta}}_{m}$ in terms of the elementary symmetric functions in the variables $\widetilde{\boldsymbol{\Gamma}}_{i}=\left(\tilde{\gamma}_{i, 1}, \ldots, \tilde{\gamma}_{i, \lambda_{i}}\right)$ with $i=1, \ldots$, $m$. For example,

$$
\sum_{a=1}^{\lambda^{(m)}} \tilde{\theta}_{\ell, a}=\sum_{i=1}^{m} \sum_{k=1}^{\lambda_{i}} \tilde{\gamma}_{i, k}-h \sum_{1 \leqslant i<j \leqslant m}\left(\lambda_{i}-\lambda_{j}\right) \frac{\tilde{q}_{i}}{\tilde{q}_{i}-\tilde{q}_{j}}, \quad m=1, \ldots, N .
$$

Relations (10.4) define a homomorphism

$$
\mathbb{C}[\widetilde{\boldsymbol{\Theta}}]^{S_{\lambda}(1)} \times \ldots \times S_{\lambda^{(N)}} \otimes \mathbb{C}[\boldsymbol{z}] \otimes \mathbb{C}[h] \rightarrow \mathcal{H}_{T}^{\tilde{q}}\left(T^{*} \mathcal{F}_{\boldsymbol{\lambda}}\right) .
$$

Let $\left\{W_{I}\right\} \in \mathcal{H}_{T}^{\tilde{q}}\left(T^{*} \mathcal{F}_{\boldsymbol{\lambda}}\right)$ be the cohomology class represented by the image of $W_{I}(\widetilde{\boldsymbol{\Theta}} ; \boldsymbol{z})$.

Theorem 10.2. For any $i=1, \ldots, N$ and $I \in \mathcal{I}_{\boldsymbol{\lambda}}$, the following relation in $\mathcal{H}_{T}^{\tilde{q}}\left(T^{*} \mathcal{F}_{\boldsymbol{\lambda}}\right)$ holds:

$$
\begin{array}{r}
\left(\sum_{k=1}^{\lambda_{i}} \tilde{\gamma}_{i, k}-\sum_{a \in I_{i}} z_{a}\right)\left\{W_{I}\right\}=h \lambda_{i}\left(\sum_{j=1}^{i-1} \frac{\tilde{q}_{j}}{\tilde{q}_{i}-\tilde{q}_{j}}+\sum_{j=i+1}^{N} \frac{\tilde{q}_{i}}{\tilde{q}_{i}-\tilde{q}_{j}}\right)\left\{W_{I}\right\}+ \\
-h \sum_{\substack{j=1 \\
j \neq i}}^{N} \sum_{m_{1}=1}^{\lambda_{i}}\left(\sum_{\substack{m_{2}=1 \\
\ell_{i, m_{1}}<\ell_{j, m_{2}}}}^{\lambda_{j}}\left\{W_{I_{i, j ; m_{1}, m_{2}}}\right\}-\frac{\tilde{q}_{i}}{\tilde{q}_{i}-\tilde{q}_{j}} \sum_{m_{2}=1}^{\lambda_{j}}\left\{W_{\left.I_{i, j ; m_{1}, m_{2}}\right\}}\right\},\right.
\end{array}
$$

where $\ell_{i, m_{1}}, \ell_{j, m_{2}}, I_{i, j ; m_{1}, m_{2}}$ are defined in Section 4.5 .

Theorem 10.2 is proved in Section 10.4.

Example. Let $N=2, n=2, \boldsymbol{\lambda}=(1,1)$. Then

$$
\left\{W_{(\{1\},\{2\})}\right\}=\tilde{\gamma}_{1,1}-z_{2}-h, \quad\left\{W_{(\{2\},\{1\})}\right\}=\tilde{\gamma}_{1,1}-z_{1},
$$

and the quantum Pieri rules take the form

$$
\begin{aligned}
& \left(\tilde{\gamma}_{1,1}-z_{1}\right)\left\{W_{(\{1\},\{2\})}=h \frac{\tilde{q}_{1}}{\tilde{q}_{1}-\tilde{q}_{2}}\left(\left\{W_{(\{1\},\{2\})}\right\}+\left\{W_{(\{2\},\{1\})}\right\}\right)-h\left\{W_{(\{2\},\{1\})}\right\},\right. \\
& \left(\tilde{\gamma}_{1,1}-z_{2}\right)\left\{W_{(\{2\},\{1\})}\right\}=h \frac{\tilde{q}_{1}}{\tilde{q}_{1}-\tilde{q}_{2}}\left(\left\{W_{(\{2\},\{1\})}\right\}+\left\{W_{(\{1\},\{2\})}\right\}\right) .
\end{aligned}
$$


These are the same relations as in formula (10.3).

10.3. Bethe ansatz equations. The Bethe ansatz equations is the following system of algebraic equations with respect to the variables $\boldsymbol{t}$ :

$$
\prod_{k=1}^{\lambda^{(i-1)}} \frac{t_{k}^{(i-1)}-t_{j}^{(i)}-h}{t_{k}^{(i-1)}-t_{j}^{(i)}} \prod_{k=1}^{\lambda^{(i+1)}} \frac{t_{j}^{(i)}-t_{k}^{(i+1)}}{t_{j}^{(i)}-t_{k}^{(i+1)}-h} \prod_{\substack{k=1 \\ k \neq j}}^{\lambda^{(i)}} \frac{t_{j}^{(i)}-t_{k}^{(i)}-h}{t_{j}^{(i)}-t_{k}^{(i)}+h}=\frac{q_{i+1}}{q_{i}}
$$

for $i=1, \ldots, N-1, j=1, \ldots, \lambda^{(i)}$. This system can be reformulated as the system of equations:

$$
\lim _{\kappa \rightarrow 0} \frac{\Phi_{\boldsymbol{\lambda}}\left(\ldots, t_{j}^{(i)}+\kappa, \ldots ; \boldsymbol{z} ; h ; \boldsymbol{q}\right)}{\Phi_{\boldsymbol{\lambda}}(\boldsymbol{t} ; \boldsymbol{z} ; h ; \boldsymbol{q})}=1, \quad i=1, \ldots, N-1, \quad j=1, \ldots, \lambda^{(i)},
$$

see [TV1, MTV1].

Lemma 10.3. For $I \in \mathcal{I}_{\boldsymbol{\lambda}}$ and $i=1, \ldots, N-1$, let $D_{I, i}(\boldsymbol{t} ; \boldsymbol{z} ; h ; \boldsymbol{q})$ be the function defined in (7.1). Let $\check{\boldsymbol{t}}$ be a solution of the Bethe ansatz equations $(10.8)$. Then $D_{I, i}(\check{\boldsymbol{t}} ; \boldsymbol{z} ; h ; \boldsymbol{q})=0$ and the right-hand side of formula (7.2) equals zero at $\boldsymbol{t}=\check{\boldsymbol{t}}$.

Proof. If $\check{\boldsymbol{t}}$ is a solution of equations (10.8), then the second of the two factors in the righthand side of formula (4.3) equals zero at $\boldsymbol{t}=\check{\boldsymbol{t}}$.

10.4. Proof of Theorem 10.2. We have the following theorem.

Theorem 10.4. Let $\check{\boldsymbol{t}}$ be a solution of the Bethe ansatz equations (10.8). Then there exist unique polynomials $\prod_{k=1}^{\lambda_{i}}\left(u-\check{\gamma}_{i, k}\right) \in \mathbb{C}[u], i=1, \ldots, N$, such that

$$
\operatorname{det}\left(q_{i}^{m-j} \prod_{k=1}^{\lambda_{i}}\left(u-\check{\gamma}_{i, k}-h(i-j)\right)\right)_{i, j=1}^{m}=\prod_{1 \leqslant i<j \leqslant m}\left(q_{i}-q_{j}\right) \prod_{a=1}^{\lambda^{(m)}}\left(u-\check{t}_{a}^{(i)}\right)
$$

for $i=1, \ldots, N-1$, and

$$
\operatorname{det}\left(q_{i}^{N-j} \prod_{k=1}^{\lambda_{i}}\left(u-\check{\gamma}_{i, k}-h(i-j)\right)\right)_{i, j=1}^{N}=\prod_{1 \leqslant i<j \leqslant N}\left(q_{i}-q_{j}\right) \prod_{a=1}^{N}\left(u-z_{a}\right) .
$$

This is [MTV2, Theorem 7.2], which is [MV2, Proposition 7.6], which in its turn is a generalization of [MV1, Lemma 4.8].

Proof of Theorem 10.2. Formula (10.6) is obtained from formula (7.2) by several substitutions. First take $q_{i}=\tilde{q}_{i}^{-1}$ for all $i=1, \ldots, N$, substitute the variables $t_{j}^{(i)}$ by $\tilde{\theta}_{i, j}$, and replace the term $D_{I, i}$ by zero. Then write symmetric functions in the variables $\widetilde{\Theta}_{m}$ via symmetric functions in the variables $\widetilde{\boldsymbol{\Gamma}}_{i}, i=1, \ldots, m$. As a result, the expression $\sum_{j=1}^{\lambda^{(i)}} t_{j}^{(i)}-\sum_{j=1}^{\lambda^{(i-1)}} t_{j}^{(i-1)}$ becomes $\sum_{j=1}^{\lambda_{i}} \tilde{\gamma}_{i, j}-h \sum_{j=1}^{i-1}\left(\lambda_{i}-\lambda_{j}\right) \tilde{q}_{j} /\left(\tilde{q}_{i}-\tilde{q}_{j}\right)$ according to formula (10.5).

Lemma 10.3 and Theorem 10.4 mean that formula (10.6) holds for those values of $\widetilde{\boldsymbol{\Gamma}}_{1}, \ldots$, $\widetilde{\boldsymbol{\Gamma}}_{N-1}$ that come from solutions $\check{\boldsymbol{t}}$ of the Bethe ansatz equations (10.8). By [MTV2, Theorem 
7.3] of completeness of the Bethe ansatz, such values of $\widetilde{\boldsymbol{\Gamma}}_{1}, \ldots, \widetilde{\boldsymbol{\Gamma}}_{N-1}$ form a Zariski open subset of all values of $\widetilde{\boldsymbol{\Gamma}}_{1}, \ldots, \widetilde{\boldsymbol{\Gamma}}_{N-1}$ satisfying defining relations of the algebra $\mathcal{H}_{T}^{\tilde{q}}\left(T^{*} \mathcal{F}_{\boldsymbol{\lambda}}\right)$, see (10.2). This proves Theorem 10.2.

10.5. Limit $\tilde{q}_{i} / \tilde{q}_{i+1} \rightarrow 0, i=1, \ldots, N-1$, and CSM classes of Schubert cells. In the limit $\tilde{q}_{i} / \tilde{q}_{i+1} \rightarrow 0$ for all $i=1, \ldots, N-1$, the algebra $\mathcal{H}_{T}^{\tilde{q}}\left(T^{*} \mathcal{F}_{\boldsymbol{\lambda}}\right)$ turns into the algebra $H_{T}^{*}\left(T^{*} \mathcal{F}_{\boldsymbol{\lambda}}\right)$ and the classes $\left\{W_{I}\right\} \in \mathcal{H}_{T}^{\tilde{q}}\left(T^{*} \mathcal{F}_{\boldsymbol{\lambda}}\right)$ become the classes $\left[W_{I}\right] \in H_{T}^{*}\left(T^{*} \mathcal{F}_{\boldsymbol{\lambda}}\right)$. Then formula (10.6) takes the form

$$
\begin{aligned}
& \left(\sum_{k=1}^{\lambda_{i}} \gamma_{i, k}-\sum_{a \in I_{i}} z_{a}\right)\left[W_{I}\right]= \\
& =h \sum_{j=1}^{i-1} \sum_{m_{1}=1}^{\lambda_{i}} \sum_{\substack{m_{2}=1 \\
\ell_{i, m_{1}}>\ell_{j, m_{2}}}}^{\lambda_{j}}\left[W_{I_{i, j ; m_{1}, m_{2}}}\right]-h \sum_{j=i+1}^{N} \sum_{m_{1}=1}^{\lambda_{i}} \sum_{\substack{m_{2}=1 \\
\ell_{i, m_{1}}<\ell_{j, m_{2}}}}^{\lambda_{j}}\left[W_{I_{i, j ; m_{1}, m_{2}}}\right] .
\end{aligned}
$$

In particular, identities in (10.7) turn into the identities

$$
\left(\gamma_{1,1}-z_{1}\right)\left[W_{(\{1\},\{2\})}\right]=-h\left[W_{(\{2\},\{1\})}\right], \quad\left(\gamma_{1,1}-z_{2}\right)\left[W_{(\{2\},\{1\})}\right]=0 .
$$

Remark. After the substitution $h=1$, the classes $\left[W_{I}\right] \in H_{T}^{*}\left(T^{*} \mathcal{F}_{\boldsymbol{\lambda}}\right)$ can be considered as elements of the equivariant cohomology $H_{\left(\mathbb{C}^{\times}\right)^{n}}^{*}\left(\mathcal{F}_{\boldsymbol{\lambda}}\right)$. By $[\mathrm{RV}]$ these new classes $\left[W_{I}\right]_{h=1}$ are proportional to the CSM classes $\kappa_{I}$ of the corresponding Schubert cells with the coefficient of proportionality independent of the index $I$. Hence formula (10.12) induces the equivariant Pieri rules for the equivariant CSM classes:

$$
\begin{aligned}
& \left(\sum_{k=1}^{\lambda_{i}} \gamma_{i, k}-\sum_{a \in I_{i}} z_{a}\right) \kappa_{I}= \\
& =h \sum_{j=1}^{i-1} \sum_{m_{1}=1}^{\lambda_{i}} \sum_{\substack{m_{2}=1 \\
\ell_{i, m_{1}}>\ell_{j, m_{2}}}}^{\lambda_{j}} \kappa_{I_{i, j ; m_{1}, m_{2}}}-h \sum_{j=i+1}^{N} \sum_{m_{1}=1}^{\lambda_{i}} \sum_{\substack{m_{2}=1 \\
\ell_{i, m_{1}}<\ell_{j, m_{2}}}}^{\lambda_{j}} \kappa_{I_{i, j ; m_{1}, m_{2}}},
\end{aligned}
$$

see detailed definitions of the CSM classes in [RV].

11. Solutions of QUANTUM Differential EQUATIONS AND EQUivariant $K$-Theory

11.1. Solutions and equivariant $K$-theory. Introduce more variables: $y=e^{2 \pi \sqrt{-1} h / \tilde{\kappa}}$,

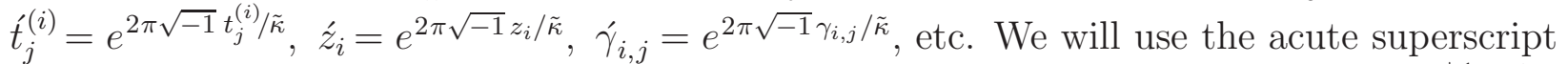
also for the corresponding collections of those variables like $\dot{\boldsymbol{\Gamma}}, \dot{\boldsymbol{t}}, \dot{\boldsymbol{z}}$. We will write $\boldsymbol{\Gamma}^{ \pm 1}, \boldsymbol{t}^{ \pm 1}$, $\dot{z}^{ \pm 1}$ for the collections extended by the inverse variables, for instance, $\dot{z}^{ \pm 1}=\left(\dot{z}_{1}^{ \pm 1}, \ldots, \dot{z}_{n}^{ \pm 1}\right)$.

Let $P$ be a Laurent polynomial in the variables $\boldsymbol{t}, \dot{\boldsymbol{z}}, y$, symmetric in $t_{1}^{(i)}, \ldots, t_{\lambda^{(i)}}^{(i)}$ for each $i=1, \ldots, N-1$. Define

$$
\widehat{\Psi}_{P}(\boldsymbol{z} ; h ; \tilde{\boldsymbol{q}} ; \tilde{\kappa})=\sum_{I \in \mathcal{I}_{\boldsymbol{\lambda}}} P\left(\dot{\Sigma}_{I}, \dot{\boldsymbol{z}}, y\right) \widehat{\Psi}_{I}(\boldsymbol{z} ; h ; \tilde{\boldsymbol{q}} ; \tilde{\kappa}),
$$


where $\widehat{\Psi}_{I}(\boldsymbol{z} ; h ; \tilde{\boldsymbol{q}} ; \tilde{\kappa})$ are given by $(9.21)$.

Lemma 11.1. The function $\widehat{\Psi}_{P}(\boldsymbol{z} ; h ; \tilde{\boldsymbol{q}} ; \tilde{\kappa})$ is a solution of both the quantum differential equations $\nabla_{\boldsymbol{\lambda}, \tilde{\boldsymbol{q}}, \tilde{\kappa}, i}^{\text {quant }} f=0, i=1, \ldots, N$, and the associated $q K Z$ difference equations.

Proof. The statement follows from Theorem 9.5.

Lemma 11.2. For a Laurent polynomial $P$ in $\dot{\boldsymbol{t}}, \dot{\boldsymbol{z}}, y$ symmetric in $t_{1}^{(i)}, \ldots, t_{\lambda^{(i)}}^{(i)}$ for each $i=1, \ldots, N-1$, the function $\widehat{\Psi}_{P}(\boldsymbol{z} ; h ; \tilde{\boldsymbol{q}} ; \tilde{\kappa})$ is holomorphic in $\tilde{\boldsymbol{q}}$ on the domain $L^{\prime \prime} \subset \mathbb{C}^{N}$ such that $\left|\tilde{q}_{i} / \tilde{q}_{i+1}\right|<1, i=1, \ldots, N-1$, and a branch of $\log \tilde{q}_{i}$ is fixed for each $i=1$, $\ldots, N$, and $\widehat{\Psi}_{P}(\boldsymbol{z} ; h ; \tilde{\boldsymbol{q}} ; \tilde{\kappa})$ is holomorphic in $\boldsymbol{z}, h$ on the domain $L^{\prime \prime \prime} \subset \mathbb{C}^{n} \times \mathbb{C}$ such that

$$
h \notin \tilde{\kappa} \mathbb{Z}_{\geqslant 0}, \quad z_{a}-z_{b}+h \notin \tilde{\kappa} \mathbb{Z}, \quad a, b=1, \ldots, n, \quad a \neq b .
$$

Proof. By the properties of $\widehat{\Psi}_{I}(\boldsymbol{z} ; h ; \tilde{\boldsymbol{q}} ; \tilde{\kappa})$, see $(9.22)$, we need only to show that the function $\widehat{\Psi}_{P}(\boldsymbol{z} ; h ; \tilde{\boldsymbol{q}} ; \tilde{\kappa})$ is regular at the hyperplanes $z_{a}-z_{b} \in \tilde{\kappa} \mathbb{Z}$. This will be done in Section 11.2 below.

Consider the equivariant $K$-theory algebra, see [RTV2, Section 2.3], [RTV3, Section 4.4],

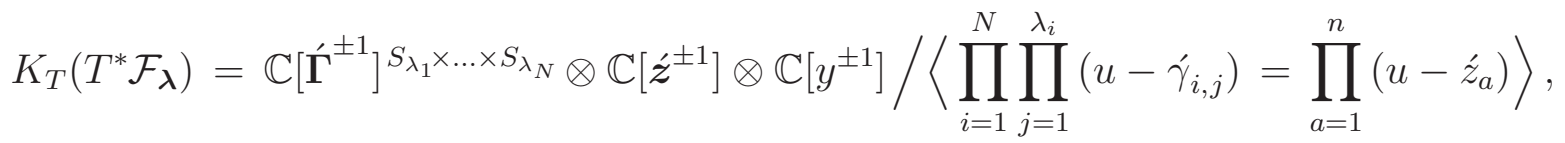

cf. (9.1). Introduce the variables $\dot{\theta}_{i, \lambda^{(i)}}, i=1, \ldots, N$. The relations

$$
\prod_{a=1}^{\lambda^{(i)}}\left(u-\dot{\theta}_{i, a}\right)=\prod_{j=1}^{i} \prod_{k=1}^{\lambda_{j}}\left(u-\dot{\gamma}_{j, k}\right), \quad i=1, \ldots, N
$$

define the epimorphism $\mathbb{C}\left[\dot{\boldsymbol{\Theta}}^{ \pm 1}\right]^{S_{\lambda^{(1)}} \times \ldots \times S_{\lambda}(N)} \otimes \mathbb{C}\left[\dot{\boldsymbol{z}}^{ \pm 1}\right] \otimes \mathbb{C}\left[y^{ \pm 1}\right] \rightarrow K_{T}\left(T^{*} \mathcal{F}_{\boldsymbol{\lambda}}\right)$. Thus the assignment $P \mapsto \widehat{\Psi}_{P}$ defines a map from $K_{T}\left(T^{*} \mathcal{F}_{\boldsymbol{\lambda}}\right)$ to the space of solutions of the quantum differential equations and the associated $q K Z$ difference equations with values in $H_{T}^{*}\left(T^{*} \mathcal{F}_{\boldsymbol{\lambda}}\right)$ extended by functions in $\boldsymbol{z}, h, \tilde{\boldsymbol{q}}$ holomorphic in the domain $L^{\prime \prime \prime} \times L^{\prime \prime}$. We evaluate below the determinant of this map.

The cohomology algebra $H_{T}^{*}\left(T^{*} \mathcal{F}_{\boldsymbol{\lambda}}\right)$ is a free module over $H_{T}^{*}(p t ; \mathbb{C})=\mathbb{C}[\boldsymbol{z}] \otimes \mathbb{C}[h]$, with a basis given by the classes of Schubert polynomials

$$
Y_{I}(\boldsymbol{\Gamma})=A_{\sigma^{I}}\left(\gamma_{1,1}, \ldots, \gamma_{1, \lambda_{1}}, \gamma_{2,1}, \ldots, \gamma_{2, \lambda_{2}}, \ldots, \gamma_{N, 1}, \ldots, \gamma_{N, \lambda_{N}}\right), \quad I \in \mathcal{I}_{\boldsymbol{\lambda}}
$$

Similarly, the algebra $K_{T}\left(T^{*} \mathcal{F}_{\boldsymbol{\lambda}}\right)$ is a free module over $K_{T}(p t ; \mathbb{C})=\mathbb{C}\left[\dot{\boldsymbol{z}}^{ \pm 1}\right] \otimes \mathbb{C}\left[y^{ \pm 1}\right]$, with a basis given by the classes of Schubert polynomials

$$
\widehat{Y}_{I}(\dot{\boldsymbol{\Gamma}})=A_{\sigma^{I}}\left(\dot{\gamma}_{1,1}, \ldots, \dot{\gamma}_{1, \lambda_{1}}, \dot{\gamma}_{2,1}, \ldots, \dot{\gamma}_{2, \lambda_{2}}, \ldots, \dot{\gamma}_{N, 1}, \ldots, \dot{\gamma}_{N, \lambda_{N}}\right), \quad I \in \mathcal{I}_{\boldsymbol{\lambda}}
$$

Both assertions are clear from Proposition A.7. 
Expand solutions of the quantum differential equation using those Schubert bases:

$$
\widehat{\Psi}_{\widehat{Y}_{I}}(\boldsymbol{\Gamma} ; \boldsymbol{z} ; h ; \tilde{\boldsymbol{q}} ; \tilde{\kappa})=\sum_{J \in \mathcal{I}_{\boldsymbol{\lambda}}} \widehat{\Psi}_{I, J}(\boldsymbol{z} ; h ; \tilde{\boldsymbol{q}} ; \tilde{\kappa}) Y_{J}(\boldsymbol{\Gamma}) .
$$

Theorem 11.3. Let $n \geqslant 2$. We have

$$
\begin{aligned}
\operatorname{det}\left(\widehat{\Psi}_{I, J}(\boldsymbol{z} ; h ; \tilde{\boldsymbol{q}} ; \tilde{\kappa})\right)_{I, J \in \mathcal{I}_{\boldsymbol{\lambda}}}= & \prod_{i=1}^{N-1} \prod_{j=i+1}^{N}\left(1-\tilde{q}_{i} / \tilde{q}_{j}\right)^{h \min \left(\lambda_{i}, \lambda_{j}\right) / \tilde{\kappa}} \prod_{i=1}^{N} \tilde{q}_{i}^{d_{\lambda, i}^{(1)} \sum_{a=1}^{n} z_{a} / \tilde{\kappa}} \times \\
& \times \prod_{\substack { a=1 \\
\begin{subarray}{c}{b=1 \\
b \neq a{ a = 1 \\
\begin{subarray} { c } { b = 1 \\
b \neq a } }\end{subarray}}^{n}\left(2 \pi \sqrt{-1} \Gamma\left(1+\frac{z_{a}-z_{b}-h}{\tilde{\kappa}}\right)\right)^{d_{\lambda}^{(2)}},
\end{aligned}
$$

where

$$
d_{\boldsymbol{\lambda}, i}^{(1)}=\frac{\lambda_{i}(n-1) !}{\lambda_{1} ! \ldots \lambda_{N} !}, \quad d_{\boldsymbol{\lambda}}^{(2)}=\frac{2(n-2) !}{\lambda_{1} ! \ldots \lambda_{N} !} \sum_{i=1}^{N-1} \sum_{j=i+1}^{N} \lambda_{i} \lambda_{j}
$$

Proof. By Lemma 11.1, the left-hand side of (11.8) solves the differential equations

$$
\left(\tilde{\kappa} \tilde{q}_{i} \frac{\partial}{\partial \tilde{q}_{i}}-\operatorname{tr}\left(X_{\boldsymbol{\lambda}, i}^{-}\left(\boldsymbol{z} ;-h ; \tilde{\boldsymbol{q}}^{-1}\right)\right)\right) \operatorname{det}\left(\widehat{\Psi}_{I, J}(\boldsymbol{z} ; h ; \tilde{\boldsymbol{q}} ; \tilde{\kappa})\right)_{I, J \in \mathcal{I}_{\boldsymbol{\lambda}}}=0, \quad i=1, \ldots, N
$$

where $X_{\boldsymbol{\lambda}, i}^{-}$are the modified dynamical Hamiltonians (9.18). Thus $\operatorname{det}\left(\widehat{\Psi}_{I, J}\right)$ equals the first two products in the right-hand side of (11.8) multiplied by a factor that does not depend on $\tilde{\boldsymbol{q}}$. The remaining factor is found by taking the limit $\tilde{q}_{i} / \tilde{q}_{i+1} \rightarrow 0$ for all $i=1, \ldots, N-1$, and applying Theorem 9.6 and Proposition A.9.

Corollary 11.4. The collection of functions $\left(\widehat{\Psi}_{\widehat{Y}_{I}}(\boldsymbol{z} ; h ; \tilde{\boldsymbol{q}} ; \tilde{\kappa})\right)_{I \in \mathcal{I}_{\boldsymbol{\lambda}}}$ is a basis of solutions of both the quantum differential equations $\nabla_{\boldsymbol{\lambda}, \tilde{\boldsymbol{q}}, \tilde{\kappa}, i}^{\text {quant }} f=0, i=1, \ldots, N$, and the associated $q K Z$ difference equations.

11.2. End of proof of Lemma 11.2. It is enough to show the regularity of $\widehat{\Psi}_{P}(\boldsymbol{z} ; h ; \tilde{\boldsymbol{q}} ; \tilde{\kappa})$ at the hyperplanes $z_{a}-z_{b} \in \tilde{\kappa} \mathbb{Z}$ assuming that $h / \tilde{\kappa}$ is real negative and sufficiently large.

For a number $A$, let $C(A) \subset \mathbb{C}$ be a parabola with the following parametrization:

$$
C(A)=\left\{\tilde{\kappa}\left(A+s^{2}-s \sqrt{-1}\right) \mid s \in \mathbb{R}\right\} .
$$

Given $\boldsymbol{z}, \tilde{\kappa}$, take $A$ such that all the points $z_{1}, \ldots, z_{n}$ are inside $C(A+N-2)$. Suppose $h / \tilde{\kappa}$ is a sufficiently large negative real so that all the points $z_{1}+h, \ldots, z_{n}+h$ are outside $C(A)$. Set

$$
\mathcal{C}_{\boldsymbol{\lambda}}(\boldsymbol{z})=(C(A))^{\times \lambda^{(1)}} \times \ldots \times(C(A+N-2))^{\times \lambda^{(N-1)}}
$$

indicating the dependence on $\boldsymbol{\lambda}$ and $\boldsymbol{z}$ explicitly. The integral (11.12) below does not depend on a particular choice of $A$. 
Lemma 11.5. For a Laurent polynomial $P$ in $\dot{\boldsymbol{t}}, \dot{\boldsymbol{z}}, y$ symmetric in $t_{1}^{(i)}, \ldots, t_{\lambda^{(i)}}^{(i)}$ for each $i=1, \ldots, N-1$, we have

$$
\widehat{\Psi}_{P}(\boldsymbol{z} ; h ; \tilde{\boldsymbol{q}} ; \tilde{\kappa})=\tilde{\kappa}^{-\lambda^{\{1\}}-2 \lambda_{\{2\}}}(-1)^{\lambda^{\{1\}}+\lambda_{\{2\}}}(2 \pi \sqrt{-1} \Gamma(-h / \tilde{\kappa}))^{-\lambda^{\{1\}}} \widetilde{\Psi}_{P}(\boldsymbol{z} ; h ; \tilde{\boldsymbol{q}} ; \tilde{\kappa})
$$$$
\widetilde{\Psi}_{P}(\boldsymbol{z} ; h ; \tilde{\boldsymbol{q}} ; \tilde{\kappa})=\frac{\widehat{\Omega}_{\boldsymbol{\lambda}}(\tilde{\boldsymbol{q}} ; \tilde{\kappa})}{\lambda^{(1) !} \ldots \lambda^{(N-1) !}} \int_{\mathcal{C}_{\boldsymbol{\lambda}}(\boldsymbol{z})} P(\boldsymbol{\boldsymbol { t }} ; \dot{\boldsymbol{z}} ; y) \Phi_{\boldsymbol{\lambda}}\left(\boldsymbol{t} ; \boldsymbol{z} ; \tilde{\boldsymbol{q}}^{-1} ;-\tilde{\kappa}\right) Q(\boldsymbol{\Gamma}) \widehat{W}(\boldsymbol{t} ; \boldsymbol{\Gamma}) d^{\lambda^{\{1\}}} \boldsymbol{t} .
$$

Proof. The integral converges provided $\left|\tilde{q}_{i} / \tilde{q}_{i+1}\right|<1$ for all $i=1, \ldots, N-1$, and a branch of $\log \tilde{q}_{i}$ is fixed for each $i=1, \ldots, N$. Evaluate the integral by residues in the following way: replace $\mathcal{C}_{\boldsymbol{\lambda}}(\boldsymbol{z})$ by $(C(A+B))^{\times \lambda^{(1)}} \times \ldots \times(C(A+B+N-2))^{\times \lambda^{(N-1)}}$, where $B \in \mathbb{R}_{\geqslant 0}$, and send $B$ to infinity. Then by (9.21), the resulting series yields formula (11.1).

The integrand in formula (11.12) is regular at the hyperplanes $z_{a}-z_{b} \in \tilde{\kappa} \mathbb{Z}$, and so does the function $\widehat{\Psi}_{P}(\boldsymbol{z} ; h ; \tilde{\boldsymbol{q}} ; \tilde{\kappa})$. Lemma 11.2 is proved.

11.3. The homogeneous case $\boldsymbol{z}=0$. The quantum differential equations $\nabla_{\boldsymbol{\lambda}, \tilde{\boldsymbol{q}}, \tilde{\kappa}, i}^{\text {quant }} f=0$ depend on $\boldsymbol{z}$ as a parameter and are well defined at $\boldsymbol{z}=0$.

For any Laurent polynomial $P$ in $\boldsymbol{t}, y$, symmetric in $t_{1}^{(i)}, \ldots, t_{\lambda^{(i)}}^{(i)}$ for each $i=1$, $\ldots, N-1$, the function $\widehat{\Psi}_{P}(0 ; h ; \tilde{q} ; \tilde{\kappa})$ is a solution of the quantum differential equations $\nabla_{\boldsymbol{\lambda}, \tilde{\boldsymbol{q}}, \tilde{\kappa}, i, \boldsymbol{z}=0}^{\text {quant }} f=0, i=1, \ldots, N$, see Lemma 11.1 .

Lemma 11.6. The function $\widehat{\Psi}_{P}(0 ; h ; \tilde{q} ; \tilde{\kappa})$ is holomorphic in $\tilde{\boldsymbol{q}}$, h provided $\left|\tilde{q}_{i} / \tilde{q}_{i+1}\right|<1$, $i=1, \ldots, N-1$, a branch of $\log \tilde{q}_{i}$ is fixed for each $i=1, \ldots, N$, and $h \notin \tilde{\kappa} \mathbb{Z}_{\geqslant 0}$.

Proof. By Lemma 11.2, we need only to show that $\widehat{\Psi}_{P}(0 ; h ; \tilde{q} ; \tilde{\kappa})$ is regular if $h \in \tilde{\kappa} \mathbb{Z}_{<0}$. We will prove that $\widehat{\Psi}_{P}(0 ; h ; \tilde{q} ; \tilde{\kappa})$ is regular if $h / \tilde{\kappa} \in \mathbb{R}_{<0}$.

If $h / \tilde{\kappa}$ is a sufficiently large negative real, write $\widehat{\Psi}_{P}(0 ; h ; \tilde{q} ; \tilde{\kappa})$ by formula $(11.12)$. Then one can replace the integration contour $\mathcal{C}_{\boldsymbol{\lambda}}(0)$ by the contour

$$
\mathcal{C}_{\lambda}^{\prime}(h, \tilde{\kappa})=(C((N-1) \varepsilon))^{\times \lambda^{(1)}} \times(C((N-2) \varepsilon))^{\times \lambda^{(2)}} \times \ldots \times(C(\varepsilon))^{\times \lambda^{(N-1)}},
$$

where $\varepsilon=h /(N \tilde{\kappa})$, without changing the integral. With the integration over $\mathcal{C}_{\lambda}^{\prime}(h, \tilde{\kappa})$, it is clear that $\widehat{\Psi}_{P}(0 ; h ; \tilde{q} ; \tilde{\kappa})$ continues to a function regular for all negative real $h / \tilde{\kappa}$.

Consider the algebras

$$
H_{\mathbb{C}^{\times}}^{*}\left(T^{*} \mathcal{F}_{\boldsymbol{\lambda}}\right)=H_{T}^{*}\left(T^{*} \mathcal{F}_{\boldsymbol{\lambda}}\right) /\langle\boldsymbol{z}=0\rangle, \quad K_{\mathbb{C}^{\times}}\left(T^{*} \mathcal{F}_{\boldsymbol{\lambda}}\right)=K_{T}\left(T^{*} \mathcal{F}_{\boldsymbol{\lambda}}\right) /\langle\dot{z}=(1, \ldots, 1)\rangle .
$$

The algebra $H_{\mathbb{C}^{\times}}^{*}\left(T^{*} \mathcal{F}_{\boldsymbol{\lambda}}\right)$ is a free module over $\mathbb{C}[h]$ and the algebra $K_{\mathbb{C}^{\times}}\left(T^{*} \mathcal{F}_{\boldsymbol{\lambda}}\right)$ is a free module over $\mathbb{C}\left[y^{ \pm 1}\right]$, with bases given by the respective classes of Schubert polynomials, see (11.5), (11.6).

Expand solutions of the quantum differential equation at $z=0$ using those Schubert bases:

$$
\widehat{\Psi}_{\widehat{Y}_{I}}(\boldsymbol{\Gamma} ; 0 ; h ; \tilde{\boldsymbol{q}} ; \tilde{\kappa})=\sum_{J \in \mathcal{I}_{\boldsymbol{\lambda}}} \widehat{\Psi}_{I, J}(0 ; h ; \tilde{\boldsymbol{q}} ; \tilde{\kappa}) Y_{J}(\boldsymbol{\Gamma})
$$


Let $d_{\boldsymbol{\lambda}}=n ! /\left(\lambda_{1} ! \ldots \lambda_{N} !\right)$. Formula $(11.8)$ at $\boldsymbol{z}=0$ takes the form

$$
\begin{aligned}
& \operatorname{det}\left(\widehat{\Psi}_{I, J}(0 ; h ; \tilde{\boldsymbol{q}} ; \tilde{\kappa})\right)_{I, J \in \mathcal{I}_{\boldsymbol{\lambda}}}= \\
& =\left(2 \pi \sqrt{-1} \Gamma\left(1-\frac{h}{\tilde{\kappa}}\right)\right)^{d_{\boldsymbol{\lambda}} \sum_{1 \leqslant i<j \leqslant N} \lambda_{i} \lambda_{j}} \prod_{i=1}^{N-1} \prod_{j=i+1}^{N}\left(1-\tilde{q}_{i} / \tilde{q}_{j}\right)^{h \min \left(\lambda_{i}, \lambda_{j}\right) / \tilde{\kappa}} .
\end{aligned}
$$

Corollary 11.7. The collection of functions $\left(\widehat{\Psi}_{\widehat{Y}_{I}}(0 ; h ; \tilde{\boldsymbol{q}} ; \tilde{\kappa})\right)_{I \in \mathcal{I}_{\boldsymbol{\lambda}}}$ is a basis of solutions of the quantum differential equations $\nabla_{\boldsymbol{\lambda}, \tilde{q}, \tilde{\kappa}, i, \boldsymbol{z}=0}^{\text {quant }} f=0, i=1, \ldots, N$.

11.4. The limit $h \rightarrow \infty$. Suppose that $\tilde{q}_{i} / \tilde{q}_{i+1}=(-h)^{-\lambda_{i}-\lambda_{i+1}} p_{i} / p_{i+1}, i=1, \ldots, N-1$, and $\tilde{q}_{N}=p_{N}$, where $p_{1}, \ldots, p_{N}$ are new variables. The limit $h \rightarrow \infty$ keeping $p_{1}, \ldots, p_{N}$ fixed corresponds to replacing the cotangent bundle $T^{*} \mathcal{F}_{\boldsymbol{\lambda}}$ by the partial flag variety $\mathcal{F}_{\boldsymbol{\lambda}}$ itself, the algebras $H_{T}^{*}\left(T^{*} \mathcal{F}_{\boldsymbol{\lambda}}\right), K_{T}\left(T^{*} \mathcal{F}_{\boldsymbol{\lambda}}\right)$ by the respective algebras $H_{A}^{*}\left(\mathcal{F}_{\boldsymbol{\lambda}}\right), K_{A}\left(\mathcal{F}_{\boldsymbol{\lambda}}\right)$, where $A \subset G L_{n}(\mathbb{C})$ is the torus of diagonal matrices, and the equivariant quantum differential equations for $T^{*} \mathcal{F}_{\boldsymbol{\lambda}}$ by the analogous equations for $\mathcal{F}_{\boldsymbol{\lambda}}$. We will discuss this limit in detail in a separate paper making here only a few remarks.

We identify $H_{A}^{*}\left(\mathcal{F}_{\boldsymbol{\lambda}}\right)$ with the subalgebra in $H_{T}^{*}\left(T^{*} \mathcal{F}_{\boldsymbol{\lambda}}\right)$ of $h$-independent elements, and $K_{A}\left(\mathcal{F}_{\boldsymbol{\lambda}}\right)$ with the subalgebra in $K_{T}\left(T^{*} \mathcal{F}_{\boldsymbol{\lambda}}\right)$ of $y$-independent elements.

The discussion of the limit $h \rightarrow \infty$ is based on Stirling's formula

$$
\frac{\Gamma(\alpha-h / \tilde{\kappa})}{\Gamma(\beta-h / \tilde{\kappa})} \sim(-h / \tilde{\kappa})^{\alpha-\beta}, \quad h \rightarrow \infty .
$$

For $\mathcal{F}_{\boldsymbol{\lambda}}$, we have the following counterparts of the master function

$$
\begin{aligned}
\Phi_{\boldsymbol{\lambda}}^{\circ}(\boldsymbol{t} ; \boldsymbol{z} ; \boldsymbol{p} ; \tilde{\kappa})=\left(e^{\pi \sqrt{-1}\left(\lambda_{N}-n\right)} p_{N}\right)^{\sum_{a=1}^{n} z_{a} / \tilde{\kappa}} \prod_{i=1}^{N-1}\left(\frac{e^{\pi \sqrt{-1}\left(\lambda_{i}-\lambda_{i+1}\right)}}{\tilde{\kappa}^{\lambda_{i}+\lambda_{i+1}}} \frac{p_{i}}{p_{i+1}}\right)^{\sum_{j=1}^{\lambda^{(i)}} t_{j}^{(i)} / \tilde{\kappa}} \times \\
\times \prod_{i=1}^{N-1} \prod_{a=1}^{\lambda^{(i)}}\left(\prod_{\substack{\lambda^{(i)} \\
b \neq a}}^{\lambda^{(i+1)}} \frac{1}{\Gamma\left(\left(t_{b}^{(i)}-t_{a}^{(i)}\right) / \tilde{\kappa}\right)} \prod_{c=1}^{\prod_{c=1}} \Gamma\left(\left(t_{c}^{(i+1)}-t_{a}^{(i)}\right) / \tilde{\kappa}\right)\right),
\end{aligned}
$$

the weight function

$$
\widehat{W}^{\circ}(\boldsymbol{t}, \boldsymbol{\Gamma})=\prod_{i=1}^{N-1} \prod_{j=i+1}^{N} \prod_{a=1}^{\lambda^{(i)}} \prod_{b=\lambda^{(i)}+1}^{\lambda^{(i+1)}}\left(t_{a}^{(i)}-\gamma_{j, b}\right)
$$

and solutions of the quantum differential equations

$$
\begin{aligned}
\widehat{\Psi}_{I}^{\circ}(\boldsymbol{z} ; \boldsymbol{p} ; \tilde{\kappa}) & =(-\tilde{\kappa})^{-\lambda^{\{1\}}-\lambda_{\{2\}}} \tilde{\kappa}^{\sum_{i=1}^{N-1} \sum_{a \in I^{i}}\left(\lambda_{i}+\lambda_{i+1}\right) z_{a} / \tilde{\kappa}} \widetilde{\Psi}_{I}^{\circ}(\boldsymbol{z} ; \boldsymbol{p} ; \tilde{\kappa}), \\
\widetilde{\Psi}_{I}^{\circ}(\boldsymbol{z} ; \boldsymbol{p} ; \tilde{\kappa}) & =\sum_{\boldsymbol{r} \in \mathbb{Z}_{\geqslant 0}^{\lambda\{1\}}} \operatorname{Res}_{\boldsymbol{t}=\Sigma_{I}+\boldsymbol{r} \tilde{\kappa}}\left(\Phi_{\boldsymbol{\lambda}}^{\circ}(\boldsymbol{t} ; \boldsymbol{z} ; \boldsymbol{p} ; \tilde{\kappa})\right) \widehat{W}^{\circ}\left(\Sigma_{I}+\boldsymbol{r} \tilde{\kappa} ; \boldsymbol{\Gamma}\right)
\end{aligned}
$$


where $I^{i}=\bigcup_{j=1}^{i} I_{j}$ and $\lambda_{\{2\}}=\sum_{1 \leqslant i<j \leqslant N} \lambda_{i} \lambda_{j}$. The series converges and defines a holomorphic function $\widehat{\Psi}_{P}^{\circ}(\boldsymbol{z} ; \boldsymbol{p} ; \tilde{\kappa})$ of $\boldsymbol{z}, \boldsymbol{p}$ on the domain in $\mathbb{C}^{n} \times \mathbb{C}^{N}$ such that a branch of $\log p_{i}$ is fixed for each $i=1, \ldots, N$, and $z_{a}-z_{b} \notin \widetilde{\kappa} \mathbb{Z}$ for all $a, b=1, \ldots, n, a \neq b$.

Set $\lambda^{\{2\}}=\sum_{i=1}^{N-1}\left(\lambda^{(i)}\right)^{2}$. As $h \rightarrow \infty$, we have $(-h)^{\lambda^{\{1\}}-\lambda^{\{2\}}} \widehat{W}(\boldsymbol{t}, \boldsymbol{\Gamma}) \rightarrow \widehat{W}^{\circ}(\boldsymbol{t}, \boldsymbol{\Gamma})$,

$$
\frac{(-h)^{\lambda^{\{2\}}-\lambda^{\{1\}}}(-h / \tilde{\kappa})^{\left(n-\lambda_{N}\right) \sum_{a=1}^{n} z_{a} / \tilde{\kappa}}}{(\Gamma(-h / \tilde{\kappa}))^{\lambda^{\{1\}}+\lambda_{\{2\}}}} \Phi_{\boldsymbol{\lambda}}\left(\boldsymbol{t} ; \boldsymbol{z} ; h ; \tilde{\boldsymbol{q}}^{-1} ;-\tilde{\kappa}\right) \rightarrow \Phi_{\boldsymbol{\lambda}}^{\circ}(\boldsymbol{t} ; \boldsymbol{z} ; \boldsymbol{p} ; \tilde{\kappa}),
$$

and

$$
\frac{\tilde{\kappa}^{\sum_{i=1}^{N-1} \sum_{a \in I^{i}}\left(\lambda_{i}+\lambda_{i+1}\right) z_{a} / \tilde{\kappa}}(-h / \tilde{\kappa})^{\left(n-\lambda_{N}\right) \sum_{a=1}^{n} z_{a} / \tilde{\kappa}}}{(\Gamma(1-h / \tilde{\kappa}))^{\lambda_{\{2\}}}} \widehat{\Psi}_{I}(\boldsymbol{z} ; h ; \tilde{\boldsymbol{q}} ; \tilde{\kappa}) \rightarrow \widehat{\Psi}_{I}^{\circ}(\boldsymbol{z} ; \boldsymbol{p} ; \tilde{\kappa}) .
$$

If $p_{i} / p_{i+1} \rightarrow 0$ for all $i=1, \ldots, N-1$, then similarly to (9.23),

$$
\begin{array}{r}
\widehat{\Psi}_{I}^{\circ}(\boldsymbol{z} ; \boldsymbol{p} ; \tilde{\kappa})=\prod_{i=1}^{N}\left(e^{\pi \sqrt{-1}\left(\lambda_{i}-n\right)} p_{i}\right)^{\sum_{a \in I_{i}} z_{a} / \tilde{\kappa}} \prod_{i=1}^{N-1} \prod_{j=i+1}^{N} \prod_{a \in I_{i}} \prod_{b \in I_{j}} \Gamma\left(1+\frac{z_{b}-z_{a}}{\tilde{\kappa}}\right) \times \\
\times\left(\Delta_{I}+\sum_{\substack{\boldsymbol{m} \in \mathbb{Z}_{\geqslant 0}^{N-1} \\
\boldsymbol{m} \neq 0}} \widehat{\Psi}_{I, \boldsymbol{m}}^{\circ}(\boldsymbol{z} ; \tilde{\kappa}) \prod_{i=1}^{N-1}\left(\frac{p_{i}}{p_{i+1}}\right)^{m_{i}}\right),
\end{array}
$$

where $\Delta_{I}(\boldsymbol{\Gamma}, \boldsymbol{z})=R_{I}(\boldsymbol{\Gamma} ; \boldsymbol{z}) / R\left(\boldsymbol{z}_{I}\right)$ is the cohomology class such that $\Delta_{I}\left(\boldsymbol{z}_{J} ; \boldsymbol{z}\right)=\delta_{I, J}$, and the classes $\widehat{\Psi}_{I, \boldsymbol{m}}^{\circ}(\boldsymbol{z} ; \tilde{\kappa})$ are rational functions in $\boldsymbol{z}, \tilde{\kappa}$, regular if $z_{a}-z_{b} \notin \tilde{\kappa} \mathbb{Z}$ for all $a, b=1$, $\ldots, n, a \neq b$.

Recall the contour $\mathcal{C}_{\boldsymbol{\lambda}}(\boldsymbol{z})$, see (11.11). Given a Laurent polynomial $P$ in the variables $\dot{\boldsymbol{t}}, \dot{\boldsymbol{z}}$, symmetric in $\dot{t}_{1}^{(i)}, \ldots, \hat{t}_{\lambda^{(i)}}^{(i)}$ for each $i=1, \ldots, N-1$, define

$$
\begin{aligned}
& \widehat{\Psi}_{P}^{\circ}(\boldsymbol{z} ; \boldsymbol{p} ; \tilde{\kappa})=\frac{(-\tilde{\kappa})^{-\lambda^{\{1\}}-\lambda_{\{2\}}} \tilde{\kappa}^{\sum_{i=1}^{N-1} \sum_{a \in I^{i}}\left(\lambda_{i}+\lambda_{i+1}\right) z_{a} / \tilde{\kappa}}}{(2 \pi \sqrt{-1})^{\lambda\{1\}}} \widetilde{\Psi}_{P}^{\circ}(\boldsymbol{z} ; \boldsymbol{p} ; \tilde{\kappa}),
\end{aligned}
$$

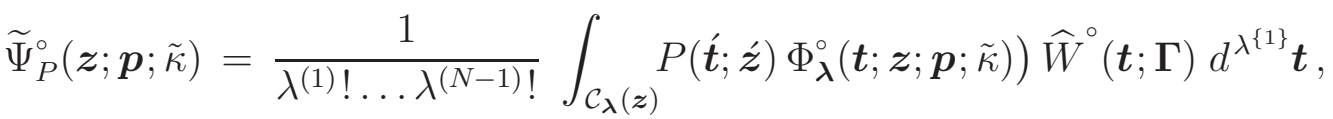

cf. (11.18). The integral converges and defines a holomorphic function $\widehat{\Psi}_{P}^{\circ}(\boldsymbol{z} ; \boldsymbol{p} ; \tilde{\kappa})$ of $\boldsymbol{z}, \boldsymbol{p}$ on the domain in $\mathbb{C}^{n} \times \mathbb{C}^{N}$ such that a branch of $\log p_{i}$ is fixed for each $i=1, \ldots, N$. Furthermore,

$$
\widehat{\Psi}_{P}^{\circ}(\boldsymbol{z} ; \boldsymbol{p} ; \tilde{\kappa})=\sum_{I \in \mathcal{I}_{\lambda}} P\left(\dot{\Sigma}_{I}, \dot{\boldsymbol{z}}\right) \widehat{\Psi}_{I}^{\circ}(\boldsymbol{z} ; \boldsymbol{p} ; \tilde{\kappa})
$$

and the assignment $P \mapsto \widehat{\Psi}_{P}^{\circ}$ defines a map from $K_{A}\left(\mathcal{F}_{\boldsymbol{\lambda}}\right)$ to the space of solutions of the quantum differential equations with values in $H_{A}^{*}\left(\mathcal{F}_{\boldsymbol{\lambda}}\right)$. 
Consider the classes $Y_{I}(\boldsymbol{\Gamma}), \widehat{Y}_{I}(\hat{\boldsymbol{\Gamma}})$ given by $(11.5),(11.6)$, and write

$$
\widehat{\Psi}_{\widehat{Y}_{I}}^{\circ}(\boldsymbol{\Gamma} ; \boldsymbol{z} ; \boldsymbol{p} ; \tilde{\kappa})=\sum_{J \in \mathcal{I}_{\boldsymbol{\lambda}}} \widehat{\Psi}_{I, J}^{\circ}(\boldsymbol{z} ; \boldsymbol{p} ; \tilde{\kappa}) Y_{J}(\boldsymbol{\Gamma}),
$$

cf. (11.7). Taking the limit $h \rightarrow \infty$ in formula (11.8) yields

$$
\operatorname{det}\left(\widehat{\Psi}_{I, J}^{\circ}(\boldsymbol{z} ; \boldsymbol{p} ; \tilde{\kappa})\right)_{I, J \in \mathcal{I}_{\boldsymbol{\lambda}}}=(2 \pi \sqrt{-1})^{d_{\boldsymbol{\lambda}} \sum_{1 \leqslant i<j \leqslant N} \lambda_{i} \lambda_{j}} \prod_{i=1}^{N} p_{i}^{d_{\lambda, i}^{(1)} \sum_{a=1}^{n} z_{a} / \tilde{\kappa}},
$$

where

$$
d_{\boldsymbol{\lambda}}=\frac{n !}{\lambda_{1} ! \ldots \lambda_{N} !}, \quad d_{\boldsymbol{\lambda}, i}^{(1)}=\frac{\lambda_{i}(n-1) !}{\lambda_{1} ! \ldots \lambda_{N} !} .
$$

Therefore, the collection of functions $\left(\widehat{\Psi}_{\widehat{Y}_{I}}^{\circ}(\boldsymbol{z} ; \boldsymbol{p} ; \tilde{\kappa})\right)_{I \in \mathcal{I}_{\boldsymbol{\lambda}}}$ is a basis of solutions of both the quantum differential equations with values in $H_{A}^{*}\left(\mathcal{F}_{\boldsymbol{\lambda}}\right)$.

\section{Appendix A. Basics on Schubert polynomials}

For references regarding Schubert polynomials, see for example [L, M].

Let $D_{1}, \ldots, D_{n-1}$ be the divided difference operators acting on functions of $x_{1}, \ldots, x_{n}$ :

$$
D_{i} f\left(x_{1}, \ldots, x_{n}\right)=\frac{f\left(x_{1}, \ldots, x_{n}\right)-f\left(x_{1}, \ldots, x_{i+1}, x_{i}, \ldots, x_{n}\right)}{x_{i}-x_{i+1}},
$$

cf. (3.7). They satisfy the nil-Coxeter algebra relations,

$$
\left(D_{i}\right)^{2}=0, \quad D_{i} D_{i+1} D_{i}=D_{i+1} D_{i} D_{i+1}, \quad D_{i} D_{j}=D_{j} D_{i}, \quad|i-j|>1 .
$$

Given $\sigma \in S_{n}$ with a reduced decomposition $\sigma=s_{i_{1}, i_{1}+1} \ldots s_{i_{j}, i_{j}+1}$, define $D_{\sigma}=D_{i_{1}} \ldots D_{i_{j}}$. For instance, $D_{\text {id }}$ is the identity operator and $D_{s_{i, i+1}}=D_{i}$. Due to relations (A.1), the operator $D_{\sigma}$ does not depend on the choice of a reduced decomposition. Moreover,

$$
D_{\sigma} D_{\tau}=D_{\sigma \tau}, \quad \text { if }|\sigma|+|\tau|=|\sigma \tau|, \quad D_{\sigma} D_{\tau}=0, \quad \text { otherwise . }
$$

Here $|\sigma|$ is the length of $\sigma$. Denote $\boldsymbol{x}_{\sigma}=\left(x_{\sigma(1)}, \ldots, x_{\sigma(n)}\right)$. Let $\sigma_{0}$ be the longest permutation, $\sigma_{0}(i)=n+1-i, i=1, \ldots, n$. Then

$$
D_{\sigma_{0}} f(\boldsymbol{x})=\prod_{1 \leqslant i<j \leqslant n}\left(x_{i}-x_{j}\right)^{-1} \sum_{\sigma \in S_{n}}(-1)^{\sigma} f\left(\boldsymbol{x}_{\sigma}\right) .
$$

The Schubert polynomials $A_{\sigma}(\boldsymbol{x}), \sigma \in S_{n}$, are defined by the rule

$$
A_{\sigma}(\boldsymbol{x})=D_{\sigma^{-1} \sigma_{0}}\left(x_{1}^{n-1} x_{2}^{n-2} \ldots x_{n-1}\right) .
$$

In particular, $A_{\sigma_{0}}=x_{1}^{n-1} x_{2}^{n-2} \ldots x_{n-1}$ and $A_{\mathrm{id}}=1$.

Proposition A.1. For any $\sigma, \tau \in S_{n}$,

$$
D_{\sigma_{0}}\left(A_{\sigma}(\boldsymbol{x}) A_{\tau \sigma_{0}}\left(\boldsymbol{x}_{\sigma_{0}}\right)\right)=(-1)^{\sigma \sigma_{0}} \delta_{\sigma, \tau} .
$$


Proposition A.2. Cauchy formula holds,

$$
\sum_{\sigma \in S_{n}}(-1)^{\sigma} A_{\sigma}(\boldsymbol{x}) A_{\sigma \sigma_{0}}(\boldsymbol{y})=\prod_{i=1}^{n-1} \prod_{j=1}^{n-i}\left(y_{i}-x_{j}\right) .
$$

For any $f \in \mathbb{C}[\boldsymbol{x}]$ and $\sigma \in S_{n}$, define $f_{\sigma} \in \mathbb{C}[\boldsymbol{x}]^{S_{n}}$ by the rule

$$
f_{\sigma}(\boldsymbol{x})=(-1)^{\sigma \sigma_{0}} D_{\sigma_{0}}\left(f(\boldsymbol{x}) A_{\sigma \sigma_{0}}\left(\boldsymbol{x}_{\sigma_{0}}\right)\right) .
$$

Proposition A.3. For any $f \in \mathbb{C}[\boldsymbol{x}]$,

$$
f(\boldsymbol{x})=\sum_{\sigma \in S_{n}} f_{\sigma}(\boldsymbol{x}) A_{\sigma}(\boldsymbol{x}) .
$$

Thus $\mathbb{C}[\boldsymbol{x}]$ is a free module over $\mathbb{C}[\boldsymbol{x}]^{S_{n}}$ of rank $n$ ! with a basis given by Schubert polynomials.

Recall the notation from Section 2.1, and $I^{\min }, I^{\max } \in \mathcal{I}_{\boldsymbol{\lambda}}$,

$$
\begin{aligned}
& I^{\min }=\left(\left\{1, \ldots, \lambda_{1}\right\}, \ldots,\left\{n-\lambda_{N}+1, \ldots, n\right\}\right), \\
& I^{\max }=\left(\left\{n-\lambda_{1}+1, \ldots, n\right\}, \ldots,\left\{1, \ldots, \lambda_{N}\right\}\right) .
\end{aligned}
$$

For $I=\left(I_{1}, \ldots, I_{N}\right) \in \mathcal{I}_{\boldsymbol{\lambda}}, I_{j}=\left\{i_{j, 1}<\ldots<i_{j, \lambda_{j}}\right\}$, define the permutations $\sigma^{I}$,

$$
\sigma^{I}(k)=i_{j, k-\lambda(j-1)}, \quad k \in I_{j}^{\min }, \quad j=1, \ldots, N,
$$

and $\sigma_{I}=\sigma^{I}\left(\sigma^{I^{\max }}\right)^{-1}$. Then $\sigma^{I}\left(I^{\min }\right)=\sigma_{I}\left(I^{\max }\right)=I$.

Let $S_{\lambda_{1}} \times \ldots \times S_{\lambda_{N}} \subset S_{n}$ be the isotropy subgroup of $I^{\mathrm{min}}$.

Lemma A.4. For any $I \in \mathcal{I}_{\boldsymbol{\lambda}}$, we have $A_{\sigma^{I}}(\boldsymbol{x}) \in \mathbb{C}[\boldsymbol{x}]^{S_{\lambda_{1}} \times \ldots \times S_{\lambda_{N}}}$.

For example, $A_{\sigma^{\max }}(\boldsymbol{x})=\prod_{a=1}^{N-1} \prod_{i \in I_{a}^{\min }} x_{i}^{N-a}$.

Proposition A.5. For any $I, J \in \mathcal{I}_{\boldsymbol{\lambda}}$,

$$
D_{\sigma^{I} \max }\left(A_{\sigma^{I}}(\boldsymbol{x}) A_{\sigma_{J}}\left(\boldsymbol{x}_{\sigma_{0}}\right)\right)=(-1)^{\sigma_{I}} \delta_{I, J} .
$$

Proposition A.6. We have,

$$
\sum_{I \in \mathcal{I}_{\boldsymbol{\lambda}}}(-1)^{\sigma^{I}} A_{\sigma^{I}}(\boldsymbol{x}) A_{\sigma_{I}}\left(\boldsymbol{y}_{\sigma_{0}}\right)=\prod_{1 \leqslant a<b \leqslant N} \prod_{i \in I_{a}^{\min }} \prod_{j \in I_{b}^{\min }}\left(y_{j}-x_{i}\right) .
$$

Proposition A.7. For any $f \in \mathbb{C}[\boldsymbol{x}]^{S_{\lambda_{1}} \times \ldots \times S_{\lambda_{N}}}$, we have

$$
f(\boldsymbol{x})=\sum_{I \in \mathcal{I}_{\boldsymbol{\lambda}}} f_{\sigma^{I}}(\boldsymbol{x}) A_{\sigma^{I}}(\boldsymbol{x}),
$$

that is, in formula (A.6), $f_{\sigma}=0$ unless $\sigma=\sigma^{I}$ for some $I \in \mathcal{I}_{\boldsymbol{\lambda}}$, and

$$
f_{\sigma^{I}}(\boldsymbol{x})=(-1)^{\sigma_{I}} D_{\sigma^{I} \max }\left(f(\boldsymbol{x}) A_{\sigma_{I}}\left(\boldsymbol{x}_{\sigma_{0}}\right)\right) .
$$


Define

$$
R_{\boldsymbol{\lambda}}(\boldsymbol{x})=\prod_{1 \leqslant a<b \leqslant N} \prod_{i \in I_{a}^{\min }} \prod_{j \in I_{b}^{\min }}\left(x_{i}-x_{j}\right)
$$

Proposition A.8. For any $f \in \mathbb{C}[\boldsymbol{x}]^{S_{\lambda_{1}} \times \ldots \times S_{\lambda_{N}}}$, we have

$$
D_{\sigma^{I m a x}} f(\boldsymbol{x})=\sum_{I \in \mathcal{I}_{\boldsymbol{\lambda}}} \frac{f\left(\boldsymbol{x}_{\sigma^{I}}\right)}{R_{\boldsymbol{\lambda}}\left(\boldsymbol{x}_{\sigma^{I}}\right)} .
$$

Proposition A.9. Let $n \geqslant 2$. Then

$$
\operatorname{det}\left(A_{\sigma^{I}}\left(\boldsymbol{x}_{\sigma^{J}}\right)\right)_{I, J \in \mathcal{I}_{\boldsymbol{\lambda}}}=\prod_{1 \leqslant i<j \leqslant n}\left(x_{j}-x_{i}\right)^{m_{\lambda}}
$$

where

$$
m_{\boldsymbol{\lambda}}=\frac{2(n-2) !}{\lambda_{1} ! \ldots \lambda_{N} !} \sum_{i=1}^{N-1} \sum_{j=i+1}^{N} \lambda_{i} \lambda_{j}
$$

\section{Appendix B. Leading Terms of Solutions and Gamma Conjecture}

The formula $(9.23)$ for the asymptotics of solutions $\left(\widehat{\Psi}_{I}(\boldsymbol{z} ; h ; \tilde{\boldsymbol{q}} ; \tilde{\kappa})\right)_{I \in \mathcal{I}_{\boldsymbol{\lambda}}}$ to the joint system of the quantum differential equations and associated qKZ difference equations reminds the statement of the gamma conjecture, see [D1, D2, KKP, GGI, GI, GZ].

The gamma conjecture [D2, GGI] is a conjecture relating the quantum cohomology of a Fano manifold $X$ with its topology. The quantum cohomology of $X$ defines a flat quantum connection over $\mathbb{C}^{\times}$in the direction of first Chern class $c_{1}(X)$. The connection has a regular singular point at $t=0$ and an irregular singular point at $t=\infty$. The connection has a distinguished (multivalued) flat section $J_{X}(t)$ defined by Givental in [Gi1] and called the J-function. Under certain assumptions, the limit of the J-function:

$$
A_{X}:=\lim _{t \rightarrow \infty} \frac{J_{X}(t)}{\left\langle[\mathrm{pt}], J_{X}(t)\right\rangle} \in H^{*}(x)
$$

exists and defines the principal asymptotic class $A_{X}$ of $X$. The gamma conjecture says that $A_{X}$ equals the gamma class $\hat{\Gamma}_{X}$ of the tangent bundle of $X$.

The gamma class of a holomorphic vector bundle $E$ over a topological space $X$ is the multiplicative characteristic class, in the sense of Hirzebruch, associated to the power series expansion $\Gamma(1+x)=1-\gamma x+\frac{\gamma^{2}+\zeta(2)}{2} x^{2}+\ldots$ of the gamma function at 1 , where $\gamma$ is the Euler constant and $\zeta(2)$ is the value at 2 of the zeta function. In other words, the gamma class is the function that associates to a holomorphic bundle $E$ over $X$ the cohomology class $\hat{\Gamma}(E)=\prod_{i} \Gamma\left(1+\tau_{i}\right) \in H^{*}(X ; \mathbb{R})$, where the total Chern class of $E$ has the formal factorization $c(E)=\prod_{i}\left(1+\tau_{i}\right)$ with the Chern roots $\tau_{i}$ of degree 2 . If $E$ is the tangent bundle of $X$, we write $\hat{\Gamma}_{X}$ for $\hat{\Gamma}(E)$. Its terms of degree $\leqslant 3$ are given by the formula

see $[\mathrm{GZ}]$.

$$
\begin{aligned}
\hat{\Gamma}(E)= & 1-\gamma c_{1}+\left(-\zeta(2) c_{2}+\frac{\zeta(2)+\gamma^{2}}{2} c_{1}^{2}\right) \\
& +\left(-\zeta(3) c_{3}+(\zeta(3)+\gamma \zeta(2)) c_{1} c_{2}-\frac{2 \zeta(3)+3 \gamma \zeta(2)+\gamma^{3}}{6} c_{1}^{3}\right)+\ldots
\end{aligned}
$$


Consider the equivariant gamma class of $T^{*} \mathcal{F}_{\boldsymbol{\lambda}}$,

$$
\widehat{\Gamma}_{T^{*} \mathcal{F}_{\boldsymbol{\lambda}}}=\prod_{i=1}^{N-1} \prod_{j=i+1}^{N} \prod_{a=1}^{\lambda_{i}} \prod_{b=1}^{\lambda_{j}} \Gamma\left(1+\gamma_{j, b}-\gamma_{i, a}\right) \Gamma\left(1+\gamma_{i, a}-\gamma_{j, b}-h\right) .
$$

cf. (9.2), and the equivariant first Chern classes $c_{1}\left(E_{i}\right)=\sum_{a=1}^{\lambda_{i}} \gamma_{i, a}, i=1, \ldots, N$, of the vector bundles $E_{i}$ over $T^{*} \mathcal{F}_{\boldsymbol{\lambda}}$ with fibers $F_{i} / F_{i-1}$, see $(9.3)$. Theorem 9.6 can be reformulated as follows.

Theorem B.1 (Gamma theorem for $T^{*} \mathcal{F}_{\boldsymbol{\lambda}}$ ). For $\kappa=1$, the leading term of the asymptotics of the q-hypergeometric solutions $\left(\widehat{\Psi}_{I}(\boldsymbol{z} ; h ; \tilde{\boldsymbol{q}} ; \tilde{\kappa})\right)_{I \in \mathcal{I}_{\lambda}}$ in $(9.23)$ is the product of the equivariant gamma class of $T^{*} \mathcal{F}_{\boldsymbol{\lambda}}$ and the exponentials of the equivariant first Chern classes of the associated vector bundles $E_{1}, \ldots, E_{N}$ :

$$
\hat{\Gamma}_{T^{*} \mathcal{F}_{\lambda}} \prod_{l=1}^{N}\left(e^{\pi \sqrt{-1}\left(\lambda_{i}-n\right)} \tilde{q}_{i}\right)^{c_{1}\left(E_{i}\right)} .
$$

Similarly formula (11.19) can be reformulated as follows.

Theorem B.2 (Gamma theorem for $\mathcal{F}_{\boldsymbol{\lambda}}$ ). For $\kappa=1$, the leading term of the asymptotics of the q-hypergeometric solutions $\left(\widehat{\Psi}_{I}^{\circ}(\boldsymbol{z} ; \boldsymbol{p} ; \tilde{\kappa})\right)_{I \in \mathcal{I}_{\boldsymbol{\lambda}}}$ in $(11.18)$ is the product of the equivariant gamma class of $T^{*} \mathcal{F}_{\boldsymbol{\lambda}}$ and the exponentials of the equivariant first Chern classes of the associated vector bundles $E_{1}, \ldots, E_{N}$ :

$$
\hat{\Gamma}_{\mathcal{F}_{\lambda}} \prod_{l=1}^{N}\left(e^{\pi \sqrt{-1}\left(\lambda_{i}-n\right)} p_{i}\right)^{c_{1}\left(E_{i}\right)}
$$

Example. Let $N=2, n=2, \boldsymbol{\lambda}=(1,1)$. For $\tilde{\kappa}=1$, the leading term of the asymptotics of the $q$-hypergeometric solutions for $T^{*} P^{1}$ is the class

$$
\left(e^{-\pi \sqrt{-1}} \tilde{q}_{1}\right)^{\gamma_{1,1}}\left(e^{-\pi \sqrt{-1}} \tilde{q}_{2}\right)^{\gamma_{2,1}} \Gamma\left(1+\gamma_{2,1}-\gamma_{1,1}\right) \Gamma\left(1+\gamma_{1,1}-\gamma_{2,1}-h\right)
$$

and the leading term of the asymptotics of the $q$-hypergeometric solutions for $P^{1}$ is the class

$$
\left(e^{-\pi \sqrt{-1}} p_{1}\right)^{\gamma_{1,1}}\left(e^{-\pi \sqrt{-1}} p_{2}\right)^{\gamma_{2,1}} \Gamma\left(1+\gamma_{2,1}-\gamma_{1,1}\right)
$$

\section{REFERENCES}

[BMO] A. Braverman, D. Maulik, A. Okounkov, Quantum cohomology of the Springer resolution, Preprint (2010), 1-35, arXiv:1001.0056

[D1] B. A. Dubrovin, Geometry and analytic theory of Frobenius manifolds, In Proceedings of the International Congress of Mathematicians, Vol. II (Berlin, 1998), 315326, arXiv:math/9807034

[D2] B. A Dubrovin, Quantum Cohomology and Isomonodromic Deformation. Lecture at Recent Progress in the Theory of Painlevé Equations: Algebraic, asymptotic and topological aspects, Strasbourg, November 2013. 
[FR] I. Frenkel, N. Reshetikhin, Quantum affine algebras and holonomic difference equations, Comm. Math. Phys. 146 (1992), no. 1, 1-60

[GGI] S. Galkin, V. Golyshev, H. Iritani, Gamma classes and quantum cohomology of Fano manifolds: gamma conjectures, Duke Math. J. 165 (2016), no. 11, 2005-2077, arXiv:1404.6407

[GI] S. Galkin, H. Iritani, Gamma conjecture via mirror symmetry, arXiv:1508.00719, 143

[Gi1] A. Givental, Equivariant Gromov-Witten invariants, Internat. Math. Res. Notices (1996), no. 13, 613-663

[Gi2] A. Givental, Stationary phase integrals, quantum Toda lattices, flag manifolds and the mirror conjecture, Topics in singularity theory, AMS Transl. Ser. 2180 (1997), $103-115$

[Gi3] A. Givental, Gromov-Witten invariants and quantization of quadratic Hamiltonians, Mosc. Math. J. 1 (2001), no. 4, 551-568

[GRTV] V. Gorbounov, R. Rimányi, V. Tarasov, A.Varchenko, Quantum cohomology of the cotangent bundle of a flag variety as a Yangian Bethe algebra, Journal of Geometry and Physics (2013), pp. 56-86, DOI: 10.1016/j.geomphys.2013.07.006

[GZ] V. Golyshev, D. Zagier, Proof of the gamma conjecture for Fano manifolds of Picard rank 1, preprint at

http://people.mpim-bonn.mpg.de/zagier/files/tex/GammaConjecture/GammaConj.pdf

[KKP] L. Katzarkov, M. Kontsevich, T. Pantev, Hodge theoretic aspects of mirror symmetry, arXiv:0806.0107, 1-124

[L] A. Lascoux, Polynomials, 2013, http://www-igm.univ-mlv.fr/ al/ARTICLES/CoursYGKM.pdf

[M] I. Macdonald, Notes on Schubert polynomials Département de mathématiques et d'informatique, Université de Québec á Montréal, 1991, ISBN 2892760860

[MO] D. Maulik, A. Okounkov, Quantum Groups and Quantum Cohomology, Preprint (2012), 1-276, arXiv:1211.1287

[MTV1] E. Mukhin, V.Tarasov, A.Varchenko, Bethe eigenvectors of higher transfer matrices, J. Stat. Mech. (2006), no. 8, P08002, 1-44

[MTV2] E. Mukhin, V.Tarasov, A.Varchenko, Spaces of quasi-exponentials and representations of the Yangian $Y\left(\mathfrak{g l}_{N}\right)$, Transf. Groups, 19 (2014), no. 2, 1-27, doi:10.1007/s00031-014-9275-8

[MV1] E. Mukhin, A.Varchenko, Solutions to the XXX type Bethe ansatz equations and flag varieties, Cent. Eur. J. Math., 1, no. 2 (2003), 238-271

[MV2] E. Mukhin, A.Varchenko, Quasi-polynomials and the Bethe Ansatz, Geometry \& Topology Monographs 13, Groups, homotopy and configuration spaces (Tokyo 2005), in honour of Fred Cohen, (2008), 385-420

[N1] H. Nakajima, Instantons on ALE spaces, quiver varieties, and Kac-Moody algebras, Duke Math. J. 76 (1994), no. 2, 365-416

[N2] H. Nakajima, Quiver varieties and Kac-Moody algebras, Duke Math. J. 91 (1998), no. 3, 515-560

[RTV1] R. Rimanyi, V.Tarasov, A.Varchenko, Partial flag varieties, stable envelopes and weight functions, Quantum Topology 6 (2015), no. 2, 333-364, DOI 10.4171/QT/64 
[RTV2] R. Rimanyi, V. Tarasov, A. Varchenko, Trigonometric weight functions as $K-$ theoretic stable envelope maps for the cotangent bundle of a flag variety, Journal of Geometry and Physics, 94 (2015), 81-119, doi:10.1016/j.geomphys.2015.04.002

[RTV3] R. Rimanyi, V. Tarasov, A. Varchenko, Elliptic and K-theoretic stable envelopes and Newton polytopes, arXiv:1705.09344, 1-35

[RV] R. Rimanyi, A.Varchenko, Equivariant Chern-Schwartz-MacPherson classes in partial flag varieties: interpolation and formulae, arXiv:1509.09315, 1-9

[TV1] V.Tarasov, A.Varchenko, Jackson Integral Representations for Solutions to the Quantized Knizhnik-Zamolodchikov Equation, St. Petersb. Math. J. 6, no. 2 (1994), $275-313$

[TV2] V.Tarasov and A.Varchenko, Duality for Knizhnik-Zamolodchikov and dynamical equations, Acta Appl. Math. 73 (2002), no. 1-2, 141-154

[TV3] V.Tarasov and A.Varchenko, Geometry of q-hypergeometric functions as a bridge between Yangians and quantum affine algebras, Invent. Math. 128 (1997), no. 3, $501-588$

[TV4] V.Tarasov and A.Varchenko, Combinatorial formulae for nested Bethe vectors, SIGMA Symmetry Integrability Geom. Methods Appl. 9 (2013), Paper 048, 1-28

[TV5] V.Tarasov and A.Varchenko, Hypergeometric solutions of the quantum differential equation of the cotangent bundle of a partial flag variety, Cent. Eur. J. Math. 12 (2014), no. 5, 694-710 